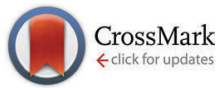

Cite this: DOI: 10.1039/c6sm02252a

\title{
Combining cell-based hydrodynamics with hybrid particle-field simulations: efficient and realistic simulation of structuring dynamics
}

\author{
G. J. A. Sevink, ${ }^{\star a}$ F. Schmid, ${ }^{b}$ T. Kawakatsu ${ }^{c}$ and G. Milano ${ }^{d}$
}

We have extended an existing hybrid MD-SCF simulation technique that employs a coarsening step to enhance the computational efficiency of evaluating non-bonded particle interactions. This technique is conceptually equivalent to the single chain in mean-field (SCMF) method in polymer physics, in the sense that non-bonded interactions are derived from the non-ideal chemical potential in self-consistent field (SCF) theory, after a particle-to-field projection. In contrast to SCMF, however, MD-SCF evolves particle coordinates by the usual Newton's equation of motion. Since collisions are seriously affected by the softening of non-bonded interactions that originates from their evaluation at the coarser continuum level, we have devised a way to reinsert the effect of collisions on the structural evolution. Merging MD-SCF with multi-particle collision dynamics (MPCD), we mimic particle collisions at the level of computational cells and at the same time properly account for the momentum transfer that is important for a realistic system evolution. The resulting hybrid MD-SCF/MPCD method was validated for a particular coarse-grained model of phospholipids in aqueous solution, against reference full-particle simulations and the original MD-SCF model. We additionally implemented and tested an alternative and more isotropic finite difference gradient. Our results show that efficiency is improved by merging MD-SCF with MPCD, as properly accounting for hydrodynamic interactions considerably speeds up the phase separation dynamics, with negligible additional computational costs compared to efficient MD-SCF. This new method enables realistic simulations of large-scale systems that are needed to investigate the applications of selfassembled structures of lipids in nanotechnologies.

www.rsc.org/softmatter

Received 4th October 2016 Accepted 3rd January 2017

DOI: $10.1039 / \mathrm{c} 6 \mathrm{sm} 02252 \mathrm{a}$ degrees of freedom in the representation, meaning that the maximum size is fixed by the chosen resolution. Any realistic computational investigation of a multi-scale phenomenon should thus make an inspired choice to sacrifice either one of them to some extent.

Historically, the community studying emergent behaviour in heterogeneous biological/chemical systems has valued resolution over size, and primarily relied on classical molecular simulation. The class of models to which classical all-atom molecular dynamics (AA-MD) belongs - known as particlebased simulations - has the benefit that chemical detail directly translates into specific molecular fragments, atoms in $\mathrm{AA}-\mathrm{MD}^{1,2}$ or small groups of (heavy) atoms in coarse-grained $\mathrm{MD}(\mathrm{CGMD})^{3}$ and dissipative particle dynamics (DPD), ${ }^{4}$ all in full agreement with standard concepts in chemical research. Nevertheless, the necessity of calculating pair interactions for each small (femtosecond) time steps, required for stable numerical integration of the equations of motion, remains a serious restriction for the upper scales of any trajectory in terms of absolute time and length scales. Particle-based approaches that go beyond the mild coarsening employed in CGMD/DPD 
have also been published recently. A prime example is the ultra coarse-grained (UCG) method, ${ }^{5,6}$ which systematically represents complete molecular domains by only a small number of degrees of freedom. Methods like UCG are more particular than the available finer-grained models in the sense that they rely on the development of molecule-specific forcefields prior to any application. Although this brings along a substantial effort compared to the tabulated CGMD force fields of, for instance, Martini, ${ }^{7}$ they are particularly advantageous for studying self-assembly and dynamics in systems containing proteins and other molecules that feature substantial internal (secondary) structure.

On the other side of the spectrum is a class of continuum or field-based methods that trade resolution for efficiency, by replacing individual particles, and thus pair interactions, by particle concentration fields and mean-field interactions. Molecular field theories like self-consistent field (SCF) theory and density functional theory (DFT) are computationally superior to particle models in terms of amenable (re)structuring pathways and system sizes, and have been successfully employed to investigate mesoscopic properties of soft structured synthetic materials like block copolymers. Nevertheless, as a result of several factors - their conceptual complexity, restrictions in the underlying molecular representation, the absence of a straightforward parametrisation and the inherent loss of (molecular) detail - only a small community has adapted this type of approaches for bio-inspired simulation. The majority of this work has concentrated on understanding the energetic and kinetic factors that regulate the self-assembly kinetics and equilibrium behaviour of vesicles formed by flexible amphiphiles in an aqueous environment, see for instance ref. 8-10 for early studies, ref. 11 for a model with hydrodynamics, and ref. 12 for an early extensive review, as a minimal model for membranes/ liposomes in biology.

Structured interfaces and membranes represent a true challenge in terms of this balance between size and resolution, since they feature two very disparate dimensions - one, perpendicular to the structure, on a molecular scale and the other, along the structure, on a (collective) macroscopic scale - that should ultimately be acknowledged in any model to enable fully realistic simulation, e.g. of liposome fusion that is induced by fusogenic molecules. Cellular membranes, playing a key role in the functioning of biological systems, are prime examples of the significance of such structures in biology. The need for a computational description of membrane properties and dynamics that accounts for this inherent multi-scale nature has prompted a number of developments that go beyond the standard pure particle- or field-based treatments, and hold a promise for being capable of accounting for internal structuring, e.g. rafts in prototypical lipid bilayers, and external factors, e.g. the interplay of membranes with membrane-bound proteins and protein complexes. As molecular detail is essential, all new developments start from particlebased models.

The most popular route assumes that detail due to solvent degrees of freedom is fairly redundant and thus can be replaced by a coarsened 'effective' description. This route has the important computational advantage that sizing results in a quadratic instead of a cubic increase of the number of degrees of freedom, as a membrane, in contrast to the solvent phase, is essentially a two-dimensional structure. In the effective description, the hydrophobic action of the solvent is represented by a continuum variable (a solvent field, giving rise to an immersed boundary method $)^{13,14}$ or only by an additional potential for the lipids, often determined by a numerical fitting procedure (implicitsolvent methods). ${ }^{15-17}$ Depending on the force field (atomistic or coarse-grained), several of these effective models have been developed in recent years, see ref. 18 and 19 for recent reviews of standard and effective approaches for membrane modelling. The main drawbacks of this route are the loss of resolution in the solvent domain and, usually, the loss of momentum transfer via the solvent phase, which is after all only represented effectively. Since hydrodynamics is known to play an important role in membrane formation and remodelling, several groups have proposed and tested solutions that re-introduce mass transfer through the solvent phase by introducing proper dynamics at the continuum level, for implicit-solvent via an auxiliary solvent field (for an example, see ref. 20). Proper treatment at the boundaries, required for a consistent coupling of field-based (solvent) and particle-based dynamics (solutes), remains a delicate issue.

An alternative route, avoiding boundary issues and allowing one to retain the solvent degrees of freedom, is to replace all pair interactions by mean-field interactions - particles moving in fields generated by all others - and thus circumvent the most elaborate part of the scheme for particle evolution. The idea draws from the detailed agreement found between particle(DPD) and field-based (SCF) simulation of block copolymer phase behaviour, suggesting that these conceptually different approaches can be related and, for some well-understood cases, mapped onto each other rather consistently. ${ }^{21}$ Several groups have recently proposed such hybrid mergers, and we shortly review the key concepts. Müller and Smith initially developed their Single Chain in Mean Field (SCMF) method to go beyond a field-based Langevin model with Onsager coefficients and more realistically capture the phase separation dynamics in dense polymers and polymers/solvent mixtures. ${ }^{22,23}$ SCMF performs a Monte Carlo (MC) simulation of independent (particle) chains in an external field obtained from the density distribution generated by the ensemble of independent chains. When this distribution changes significantly, a property that is usually imposed by an update frequency, this external field is updated from the instantaneous density distribution. The same concept, i.e. representing non-bonded interactions via a particle-derived density distribution in a field-based Hamiltonian, was later used to device an efficient solvent-free simulation method for lipid membranes. ${ }^{24,25}$ Also the MD-SCF method of Milano and Kawakatsu ${ }^{26}$ uses (field-based) chemical potentials to derive intermolecular particle forces, but now for the standard Newton's equations of motion employed in CGMD. In particular, the condition of local equilibrium with respect to a slowly varying external field is satisfied at the particle level, via (Martini) CGMD, while the remainder of the SCF free 
energy, the non-ideal part which only depends on projected particle density fields, is minimised by consistent forces on particles. Hence, the calculation of pair interactions is circumvented in such approaches and direct access to the (non-ideal) free energy granted. Apart from that fact that softening enables a significantly larger time step for stable numerical integration of Newton's equations of motion, the simulation algorithm can also be parallelised efficiently, since it only considers fields and individual particle chains. ${ }^{27}$ The transferability between CGMD and MD-SCF was validated for a number of cases, including for phospholipid membranes. ${ }^{29}$

By selecting a global Andersen thermostat for the particle evolution, ${ }^{26}$ however, the advantage of retaining solvent degrees of freedom has not been fully exploited. Improving the kinetic description, such that it realistically addresses phenomena that play a key role both in the early stage of phase separation and in membrane dynamics and remodelling, would make the method stand out among competitors, and introduce the option to simulate lipid structures of truly relevant dimensions with proper resolution and dynamics. Here, we adapt the original method by coupling hybrid MD-SCF to cell-based multi-particle collision dynamics (MPCD), thereby reinserting collisions that have been removed by the introduction of soft (field-based) potentials. Although this new approach is applicable to any molecular representation, we analyse the new hybrid method for an existing lipid-water representation taken from DPD, which allows us to benefit from established mappings, and compare results to both DPD and the original MD-SCF method. The advantage compared to the original MD-SCF is that the coarsening dynamics is significantly enhanced by MPCD, while the advantage to DPD is enhanced efficiency (roughly a factor of two) and a much more direct control of fluid properties, e.g. viscosity as a function of local composition, than DPD. ${ }^{30,31}$

\section{General theory}

\section{MD-SCF}

The MD-SCF method follows the approach of SCF in treating the inter- and intramolecular interactions in a particle-based molecular description separately. In particular, the Hamiltonian of a system composed of $M$ molecules is split into

$$
\hat{H}(\Gamma)=\hat{H}_{0}(\Gamma)+\hat{W}(\Gamma)
$$

where $\Gamma$ specifies a point in phase space, i.e. a set of positions of all particles (atoms or chemical fragments) in the system, and $\hat{\imath}$ indicates that the associated physical quantity is a function of all microscopic states described by $\Gamma$. The first term in (1), $\hat{H}_{0}(\Gamma)$, is the Hamiltonian of a reference ideal system of noninteracting chains. Instead of the usual Hamiltonian for Gaussian chains of SCF, it represents all intra-molecular - bond, angle, torsion and non-bonded (Lennard-Jones) - interactions in a particle-based representation of choice. The second term, $\hat{W}(\Gamma)$, is the deviation from the reference system stemming from the intermolecular interactions. In a $N V T$ ensemble, the partition function of this system is

$$
Z=\frac{1}{M !} \int \mathrm{d} \Gamma \mathrm{e}^{-\beta\left[\hat{H}_{0}(\Gamma)+\hat{W}(\Gamma)\right]}
$$

with the usual definition of $\beta=1 / k_{\mathrm{B}} T$.

From a microscopic point of view, the density distribution of particles can be defined as

$$
\hat{\phi}(r, \Gamma)=\sum_{p=1}^{M} \sum_{i=0}^{S(p)} \delta\left(\mathbf{r}-\mathbf{r}_{i}^{(p)}\right)
$$

where $S(p)$ is the number of particles in the $p$-th molecule and $\mathbf{r}_{i}^{(p)}$ is the center of mass of the $i$-th particle in the $p$-th molecule. For the particular lipid-water system considered further on, $S(p)=N_{1}$ is the number of particles in a lipid chain and $S(p)=1$ for water particles. To determine the intermolecular interactions, it is assumed that

$$
\hat{W}(\Gamma)=W[\hat{\phi}(\mathbf{r}, \Gamma)]
$$

and one can, after some calculus, ${ }^{26}$ rewrite the partition function (2) as

$$
\begin{aligned}
Z= & \frac{1}{M !} \int D\{\phi(\mathbf{r})\} \\
& \times \int D\{w(\mathbf{r})\} \mathrm{e}^{-\beta\left[-\frac{M}{\beta} \ln z+W[\phi(\mathbf{r})]-\frac{i}{\beta} \int w(\mathbf{r}) \phi(\mathbf{r}) \mathrm{d} \mathbf{r}\right]},
\end{aligned}
$$

where $z$ is the single molecule partition function, and $w(\mathbf{r})$ a conjugate field of $\phi(\mathbf{r})$. In particular, the external potential $\mathcal{V}(\mathbf{r})=(i / \beta) w(\mathbf{r})$ has been made explicit to clarify the $w$-dependence of the integrand. Next, one employs the saddle point or stationary phase method to approximate the partition function, providing the relations

$$
\mathcal{V}(\mathbf{r})=\frac{\delta W[\phi(\mathbf{r})]}{\delta \phi(\mathbf{r})}
$$

$$
\phi(\mathbf{r})=-\frac{M}{\beta z} \frac{\delta z}{\delta \mathcal{V}(\mathbf{r})}=\langle\hat{\phi}(\mathbf{r}, \Gamma)\rangle
$$

Equations (1)-(6) simply follow the original derivation of Milano and Kawakatsu; ${ }^{26}$ the extension to the general case of different particle types (labeled by $K$ ) is straightforward. The interaction term $W[\phi(\mathbf{r})]$ is yet unspecified, and we follow the original approach in considering the standard non-ideal free energy of SCF

$$
\begin{aligned}
W\left[\left\{\phi_{K}(\mathbf{r})\right\}\right]= & \int_{V}\left[\frac{k_{\mathrm{B}} T}{2} \sum_{K K^{\prime}} \chi_{K K^{\prime}} \phi_{K}(\mathbf{r}) \phi_{K^{\prime}}(\mathbf{r})\right. \\
& \left.+\frac{\kappa_{\mathrm{H}}}{2}\left(\sum_{K} \phi_{K}(\mathbf{r})-\phi_{0}\right)^{2}\right] \mathrm{d} \mathbf{r}
\end{aligned}
$$

where $V$ is the simulation volume, $\phi_{K}(\mathbf{r})$ is the coarse-grained density of particle type $K$ at position $\mathbf{r}$ and $\chi_{K K^{\prime}}=\chi_{K^{\prime} K}$ is the mean-field interaction strength of a particle of type $K$ with the surrounding density field for particle type $K^{\prime}$ known 
as the Flory-Huggins ( $\mathrm{FH})$ parameters. The second term in (7) is the Helfand penalty function that allows for small variation of the total density field around a constant (background) value $\phi_{0}$, allowing one to control the excluded volume interactions. The strength $\kappa_{\mathrm{H}}=1 / \kappa$, labeled $\mathrm{H}$ to denote its Helfand origin, sets the tolerated deviation from the constant total number density of segments $\phi_{0}$. We selected $\kappa_{\mathrm{H}}$ over the original incompressibility parameter, $\kappa$ in ref. 26 , to relate our results in the remainder more directly to previous work based on an alternative hybrid description for the same lipid model. ${ }^{14}$

For completeness, we note that an alternative and legitimate option is to simply start from the Hamiltonian (7) for intermolecular interactions on a coarse level, thereby avoiding any misconceptions that may appear in the use of the intermediate density distribution (3). In combination with an appropriate smoothing procedure for the particle-to-field mapping, which we will address below, this Hamiltonian defines the hybrid model.

One can now easily determine the mean field (chemical) potential $\mathcal{V}_{K}(\mathbf{r})$ as

$$
\begin{aligned}
\mathcal{V}_{K}(\mathbf{r}) & =\frac{\delta W\left[\left\{\phi_{K}(\mathbf{r})\right\}\right]}{\delta \phi_{K}(\mathbf{r})} \\
& =k_{\mathrm{B}} T \sum_{K^{\prime}} \chi_{K K^{\prime}} \phi_{K^{\prime}}(\mathbf{r})+\kappa_{\mathrm{H}}\left(\sum_{K} \phi_{K}(\mathbf{r})-\phi_{0}\right)
\end{aligned}
$$

For instance, for the $\mathrm{H}_{3}\left(\mathrm{C}_{4}\right)_{2} / \mathrm{W}$ (lipid/water) representation of ref. 44 considered here, with three particle types $(K \in\{\mathrm{H}, \mathrm{C}, \mathrm{W}\})$, the mean field potentials are given by

$$
\begin{aligned}
\mathcal{V}_{K}(\mathbf{r})= & k_{\mathrm{B}} T\left[\chi_{K \mathrm{H}} \phi_{\mathrm{H}}(\mathbf{r})+\chi_{K \mathrm{C}} \phi_{\mathrm{C}}(\mathbf{r})+\chi_{K \mathrm{~W}} \phi_{\mathrm{W}}(\mathbf{r})\right] \\
& +\kappa_{\mathrm{H}}\left[\phi_{\mathrm{H}}(\mathbf{r})+\phi_{\mathrm{C}}(\mathbf{r})+\phi_{\mathrm{W}}(\mathbf{r})-\phi_{0}\right]
\end{aligned}
$$

and the resulting forces $\mathbf{F}_{K}(\mathbf{r})=-\nabla_{\mathbf{r}} \mathcal{V}_{K}(\mathbf{r})$ on a particle of type $K$ at position $\mathbf{r}$

$$
\begin{aligned}
\mathbf{F}_{K}(\mathbf{r})= & -\left(k_{\mathrm{B}} T \chi_{K \mathrm{H}}+\kappa_{\mathrm{H}}\right) \nabla_{\mathbf{r}} \phi_{\mathrm{H}}(\mathbf{r}) \\
& -\left(k_{\mathrm{B}} T \chi_{K \mathrm{C}}+\kappa_{\mathrm{H}}\right) \nabla_{\mathbf{r}} \phi_{\mathrm{C}}(\mathbf{r}) \\
& -\left(k_{\mathrm{B}} T \chi_{K \mathrm{~W}}+\kappa_{\mathrm{H}}\right) \nabla_{\mathbf{r}} \phi_{\mathrm{W}}(\mathbf{r})
\end{aligned}
$$

The density fields $\phi_{K}(\mathbf{r})$ are defined on a uniform cubic grid with spacing $l$. There is some flexibility of choice in the particle-to-field projection, and we assign particle fractions to neighbouring grid points using properly normalised Gaussians instead of the previous employed trilinear functions. This projection generates a less fluctuating field for the force calculation. Although these particle forces could be determined via a variational approach, we rely on the original grid-based method that calculates field gradients numerically using a staggered grid (see the appendix for more details) and we additionally consider another, more isotropic discrete gradient operator. In the remainder, we assume that the mass $m$ for all particles is the same and set it to unity. Moreover, we also set $k_{\mathrm{B}} T=1$.
Dynamics. As usual, new configurations are obtained by integrating the equations of motion from time $t$ to $t+\Delta t$ by a velocity Verlet (VV) algorithm

$$
\begin{gathered}
\mathbf{r}_{i}(t+\Delta t)=\mathbf{r}_{i}(t)+\mathbf{v}_{i}(t) \Delta t+\frac{1}{2} \mathbf{f}_{i}(t) \Delta t^{2} \\
\mathbf{v}_{i}(t+\Delta t)=\mathbf{v}_{i}(t)+\frac{1}{2}\left(\mathbf{f}_{i}(t)+\mathbf{f}_{i}(t+\Delta t)\right) \Delta t
\end{gathered}
$$

for all particles in the system, where $\mathbf{r}_{i}$ and $\mathbf{v}_{i}$ represent the position and velocity of the $i$ th particle, and the force $\mathbf{f}_{i}$ is the sum of the intra-molecular forces and the mean field force, i.e. $\mathbf{f}_{i}(\mathbf{r})=\mathbf{f}_{i}^{\text {intra }}(\mathbf{r})+\mathbf{F}_{K}(\mathbf{r})$, with $K$ the type of particle $i$. For water particles, the absence of intra-molecular forces is reflected in $\mathbf{f}_{i}(\mathbf{r})=\mathbf{F}_{\mathrm{W}}(\mathbf{r})$, meaning that solvent particles only experience forces due to the density fields.

Although the MD-SCF model is essentially off-lattice, molecules only interact via smoothed potentials that are defined on a (coarse) regular lattice. As a consequence, the contribution of particle collisions to the relaxation dynamics is largely underestimated, especially when the gradient terms practically vanish, for instance at large densities $\phi_{0}$. In the original MD-SCF approach, ${ }^{26}$ the $\mathrm{VV}$-algorithm was combined with a (local) Andersen thermostat to mimic the effect of particle collisions and maintain a constant (kinetic) simulation temperature, by replacing the velocity of a number of randomly selected particles by a velocity drawn from a Maxwell distribution. However, such a local thermostat does not properly account for momentum transfer, as a result of a violation of Galilean invariance, ${ }^{30,32}$ and thus the relaxation dynamics is less realistic and generally very slow (see results section for examples). In principle, we could introduce Galilean invariance by another choice of the thermostat, see, for instance, the one developed in ref. 30, but this will not fundamentally resolve the discussed smoothing effect on collisions, and the computational penalty is considerable, as such thermostats rely on computationally expensive pairwise velocity-difference calculations.

\section{Multi-particle collision dynamics (MPCD)}

The class of algorithms known as MPCD or stochastic rotation dynamics (SRD), a name that was used when the method was first formulated, combines discrete time with continuous space, and represents a fluid by point-like particles that undergo ballistic motion

$$
\mathbf{r}_{i}(t+\delta t)=\mathbf{r}(t)+\mathbf{v}_{i}(t) \delta t
$$

with $\delta t$ a well-chosen time increment (streaming step). Particles only interact during collision steps, when momentum exchange takes place. Several energy and momentum conserving collision schemes have been proposed ${ }^{36}$ and share the property that, instead of pairwise velocity differences, only the center of mass velocity in each cell of a coarse grid needs to be calculated for a velocity update. It was shown that MPCD is Galilean invariant, provided that all cell positions are shifted by a well-chosen random vector prior to each collision step. ${ }^{35}$ Consequently, MPCD can be seen as a very simple and efficient numerical approach for solving the Navier-Stokes equations 
and describing the hydrodynamics of viscous systems on a mesoscopic level.

Focussing on the collision step, the three-dimensional simulation volume $V$ is coarse-grained into cells of size $a \times a \times a$, with $a$ setting the resolution of the hydrodynamics. After shifting particle positions, by a random vector $\mathbf{t}=\left(t_{x}, t_{y}, t_{z}\right)^{T}$ with components $t_{j} \in[-a / 2, a / 2](j=x, y, z)$ taken from a uniform distribution, ${ }^{35}$ particles are sorted to the cells and, for each cell, a center of mass velocity for the $n_{\mathrm{c}}$ particles in the cell

$$
\mathbf{v}_{\mathrm{cm}}=\frac{1}{n_{\mathrm{c}}} \sum_{j=1}^{n_{\mathrm{c}}} \mathbf{v}_{j}
$$

is calculated. The velocity update is given by

$$
\mathbf{v}_{i}(t+\delta t)=\mathbf{v}_{\mathrm{cm}}(t)+\mathcal{R}\left(\mathbf{v}_{i}(t)-\mathbf{v}_{\mathrm{cm}}(t)\right),
$$

with $\mathbf{v}_{\mathrm{cm}}$ the value for the cell in which particle $i$ is located, $\delta t$ a time interval between collisions and $\mathcal{R}$ a $3 \times 3$ matrix that rotates vectors by a fixed angle $\alpha$ around an axis that is generated randomly for each cell. ${ }^{36}$ After this update, particles are shifted back using the same random vector. The total number of particles, and thus the average number of particles per cell $n_{\mathrm{s}}$, is a freedom of choice but remains constant during simulation. The algorithmic simplicity enables an analytic derivation of hydrodynamic properties in terms of parameters $n_{\mathrm{s}}, \alpha$ and $\delta t$, via the Green-Kubo relations, ${ }^{37,38}$ opening up a possibility of mapping to real systems.

Hybrid MPCD/MD. Despite its advantageous computational efficiency, standard MPCD does not apply to phase separating systems. In contrast to CGMD or DPD, where each particle represents a cluster of 3-4 solvent molecules with a base (excluded volume) repulsion in addition to non-ideal interactions, MPCD particles are point-like fictitious entities without potential-based interactions or relation to molecular detail. In other words, MPCD solvent can act as a momentum-conserving heat bath for any solute, ${ }^{39}$ where solute particles can experience bonded interactions and should be included in the collision step, but the overall description remains that of an ideal and compressible gas. In particular, the lack of explicit interactions results in unphysical particle trajectories.

Fortunately, MPCD was recently extended to include the non-ideal interactions that play a role in many systems containing both solvent and solutes. One route, which was later formally linked to an equation of state for a system containing two particle types, ${ }^{40}$ is to introduce a new set of collision rules that acknowledges the different nature of colliding particles. ${ }^{41}$ The resulting algorithm was numerically tested by considering the phase behaviour of binary mixtures, analysing the spectra of capillary wave fluctuations on a droplet, and even by simulating phase separation in a ternary surfactant mixture. ${ }^{40}$ Generalisation to many particle types, however, is not straightforward.

The second route is a hybrid MPCD/MD scheme developed and analysed by Malevanets and Kapral, ${ }^{42}$ which couples a forcefield based description for solute-solute and solute-solvent interactions to an effective MPCD description for the solventsolvent interactions. In short, the ballistic streaming step (12) is replaced by the $\mathrm{VV}$ scheme of $\mathrm{MD}$, assuming that there are no solvent-solvent interactions besides collisions, and the two descriptions evolve on a different time scale. This hybrid MPCD/MD model serves as a basis in the present study.

\section{Hybrid MD-SCF/MPCD scheme}

An attractive solution for the very weak coupling between solvents and solutes in MD-SCF is to re-introduce particle collisions via the off-grid multi-particle collision dynamics (MPCD) method, in which averaged particle velocities, calculated in lattice cells, are used to perform effective collisions between individual particles. By combining MPCD and MD-SCF, the implementation of which is discussed next, we restore the effect of particle collisions on the relaxation dynamics. At the same time, the method can be seen as a natural extension of the SRD/MPCD framework to complex and multicomponent fluids, reminiscent of the way Lattice Boltzmann (LB) was extended to complex fluids, by coupling it to a free energy functional. ${ }^{33,34}$

Our new hybrid scheme is very similar to the MPCD/MD scheme of Malevanets and Kapral, ${ }^{42}$ but differs in the handling of the solvent, which is treated on the same footing as the solute during the streaming step. In the collision step, governed by the time interval $\delta t$ of MPCD, only solvent particles collide according to the update (14). In the streaming step, associated with a smaller time step $\Delta t$, both solute and solvent evolve according to the VV scheme (12). The force acting on each solute particle contains intramolecular (bond, angle, torsion) terms as well as non-bonded (intermolecular) contributions due to other solutes and due to solvent particles. In the MPCD/MD scheme of Malevanets and Kapral, the force acting on solvent particles stems only from the solutes, which is numerically efficient but can also be seen as artificial. In the new scheme, the forces on solvent particles are simply given by $\mathbf{f}_{i}(\mathbf{r})=\mathbf{F}_{\mathrm{W}}(\mathbf{r})$ of (10). Thus, besides the 'standard' contributions due to the solutes, albeit in terms of their density fields, there is another term $-\kappa_{\mathrm{H}} \nabla_{\mathbf{r}} \phi_{\mathrm{W}}(\mathbf{r})$ (note that a standard value for $\chi_{\mathrm{ww}}=0$ ) that renders the force dependent on spatial variations of the solvent density field and stems from excluded volume interactions on the field level. This scheme reduces to MPCD/MD only for $\kappa_{\mathrm{H}}=0$. For $\kappa_{\mathrm{H}} \neq 0$, any spatial variation of the total density $\phi(\mathbf{r})=\sum_{K} \phi_{\mathrm{K}}(\mathbf{r})$ from the reference value $\phi_{0}$ will induce particle forces consistent with a reduction of this variation. Particularly in solvent-rich phases, $\phi_{\mathrm{W}} \approx \phi_{0}$, this additional force term perturbs the solvent particle dynamics. By thus combining the MPCD/MD concept with the MD-SCF Hamiltonian, we obtain a more consistent description of the hydrodynamics of complex fluids, where one no longer has to distinguish between different components of the complex fluid. In the results section, we will quantify the effect of these additional force terms on the dynamics.

Momentum conserving thermostat. Momentum conservation is exact in standard MPCD, and the hybrid MPCD/MD scheme of Malevanets and Kapral ${ }^{42}$ conserves energy, meaning that a thermostat is superfluous when kinetic energy dominates, for 
instance when solute particles interact only very weakly or when there is a strong mass misbalance between solute and solvent particles. For balanced systems, however, a thermostat may be essential for maintaining a constant temperature during simulation and, more in general, for the stability of the trajectory. This thermostat could be introduced at the MD level, for instance in the form of a Langevin-thermostat that couples to the relative velocity of the particles with respect to the mean velocity in their cell. Nevertheless, we select the Maxwell-Boltzmann scaling (MBS) thermostat, ${ }^{43}$ which leaves the total momentum of a cell unchanged, since a thermostat on the MPCD level is most efficient. In this thermostat, the relative velocities $\Delta \mathbf{v}_{i}=\mathbf{v}_{i}-\mathbf{v}_{\mathrm{cm}}$ of the particles in a collision cell are scaled by a constant $\xi$ during the collision step, with $\xi$ a particular value for each collision cell. In MBS, the factor is defined as

$$
\xi=\sqrt{\hat{E}_{\mathrm{k}} / E_{\mathrm{k}}}
$$

where $E_{\mathrm{k}}=1 / 2 \sum_{j=1}^{n_{\mathrm{c}}} m \Delta \mathbf{v}_{j}^{2}$ is the kinetic energy of the $n_{\mathrm{c}}$ particles in a cell and $\hat{E}_{\mathrm{k}}$ is selected from an appropriate distribution function for each collision cell. ${ }^{43}$ In particular, the selected $\Gamma$ distribution converges to a Gaussian function with proper values for its mean and variance when $f=3\left(n_{\mathrm{c}}-1\right)$, the number of degrees of freedom of the fluid particles in the considered collision cell, goes to infinity. Solute particles are also thermostatted during the collision step via $\mathbf{v}_{i}(t+\delta t)=\mathbf{v}_{\mathrm{cm}}(t)+\xi\left(\mathbf{v}_{i}(t)-\mathbf{v}_{\mathrm{cm}}(t)\right)$. In particular, they are subjected to a velocity scaling that leaves the total momentum per cell unchanged, but not to rotation. After each thermostatting stage, any net momentum is removed by rescaling the mean particle velocity to zero.

On Galilean invariance. We should note that even though the underlying continuum Hamiltonian (7) is Galilean invariant, the sum over all forces (10) is not strictly zero due to discretization effects, hence momentum is not strictly preserved. Strict total momentum conservation can be imposed by adding an additional constraining force (a Lagrangian parameter) in each step. We have tested this and found that this may affect the results if we use the gradient operator introduced by Milano and Kawakatsu, ${ }^{26}$ but not if we use a more isotropic gradient operator introduced in this paper. Furthermore, we found that discretization effects could be reduced significantly if the inertial frame of the simulation was chosen such that the discretization grid moves with a constant background speed. Details are discussed in Appendix C.

\section{Practical implementation of MD-SCF/MPCD for a lipid model}

It is good to realise that one may select any coarse-grained molecular representation in the hybrid approach. Whereas the original MD-SCF approach benefits from selecting a familiar Martini representation for which many biomolecules/solvents have been parameterised, it introduces the necessity to determine a map between the Martini force fields and effective $\mathrm{FH}$ parameters. ${ }^{26}$ For the purpose of illustration, we instead select the DPD representation of Shillcock and Lipowsky, ${ }^{44}$ i.e. lipids are represented by a $\mathrm{H}_{3}\left(\mathrm{C}_{4}\right)_{2}$ chain $\left(N_{1}=11\right.$ particles $)$, for which a mapping to FH-parameters already exists. Like in CGMD, 3-4 water molecules constitute one $\mathrm{W}$ particle. In particular, since the hybrid method represents all intermolecular interactions at a field level, the treatment of the solvent phase (as single particles) is equivalent in both representations.

We distinguish dimensionless variables from physical quantities by an asterix. As usual in DPD, ${ }^{44}$ we scale length and time as $r^{*}=r / r_{\mathrm{c}}$ and $t^{*}=t \sqrt{k_{\mathrm{B}} T / m r_{\mathrm{c}}^{2}}$, with $r_{\mathrm{c}}$ the cutoff distance of DPD. This scaling corresponds to the choice $m=1, r_{\mathrm{c}}=1$ and $k_{\mathrm{B}} T=1$ of reference units. Important $\mathrm{SRD} / \mathrm{MPCD}$ variables are the cell size $a_{\mathrm{MPCD}}=r_{\mathrm{c}}\left(a_{\mathrm{MPCD}^{*}}=1\right)$, unless specified otherwise, and mean free path $\lambda=\delta t \sqrt{k_{\mathrm{B}} T / m a_{\mathrm{MPCD}^{2}}}\left(\lambda^{*}=\delta t^{*}\right.$ for $\left.a_{\mathrm{MPCD}^{*}}=1\right)$. Initially, all $N$ particles are placed in a cuboidal system of constant volume $V=L_{x} L_{y} L_{z}$ (all sizes in units of $r_{\mathrm{c}}$ ), so that the overall particle density $\phi_{0}{ }^{*}=N / V^{*}\left(V^{*}=V / r_{\mathrm{c}}{ }^{3}\right)$ is fixed. The first lipid head $(H)$ particle and all solvent particles are usually placed at random. In setting up a simulation, choices for the density $\phi_{0}{ }^{*}$ and size $V^{*}$ provide $N=\phi_{0}{ }^{*} V^{*}$, whereas a choice for the number of lipid chains $n_{1}$ defines the number of water particles as $n_{\mathrm{w}}=N-N_{\mathrm{l}} n_{\mathrm{l}}$. All simulations start from a Maxwell-Boltzmann distribution for the particle velocities at a given (kinetic) temperature $T$, with an average kinetic energy $m\left\langle v^{2}\right\rangle=3 k_{\mathrm{B}} T$. The configurational temperature is sometimes advocated as a better measure, ${ }^{45}$ but it is based on the gradient and Laplacians of the interaction potentials, so we rely on the standard procedure of monitoring the kinetic temperature $\left\langle\left(\mathbf{v}^{*}\right)^{2}\right\rangle / 3$ instead. In all simulations, periodic boundary conditions are used. Although all simulations for lipid/water systems were repeated with 3 different noise seeds to check consistency, all reported results are for individual simulation trajectories.

The strength of the 'soft' (quadratic) DPD interaction potentials is given by the expression $a_{i j}^{*}=a_{i i}^{*}+\Delta a_{i j}^{*}$ (using $\left.a^{*}=a r_{\mathrm{c}} / k_{\mathrm{B}} T\right)$, with $a_{i i}{ }^{*}=75 / \rho_{0}{ }^{*}{ }^{21}$ For the usual density of $\rho_{0}{ }^{*}=3\left(a_{i i}{ }^{*}=25\right)$, Groot and Warren developed a mapping between the meanfield FH parameters $(\chi>2)$ and $\Delta a^{*}$ for mixtures of single particles as ${ }^{21}$

$$
\chi=(0.286 \pm 0.002) \Delta a^{*}
$$

or, for short chains,

$$
\chi=(0.306 \pm 0.003) \Delta a^{*} .
$$

Using expression (16) for the water-lipid and (17) for the lipidlipid interaction to convert the $\Delta a^{*}$ for the chosen DPD parameters, i.e. $\Delta a_{K K^{*}}=0$ for $\mathrm{K} \in\{\mathrm{H}, \mathrm{C}, \mathrm{W}\}, \Delta a_{\mathrm{HC}^{*}}=25, \Delta a_{\mathrm{HW}^{*}}=10$ and $\Delta a_{\mathrm{CW}^{*}}=50,{ }^{44}$ we obtain the values shown in Table 1. Matching the equation of state for the pressure, a value for the

Table 1 The dimensionless interaction $\chi_{K K^{\prime}}$ for particles of type $K$ interacting with a density field due to particle of type $K^{\prime}$. Mean-field interactions between the same particle types are zero by definition

\begin{tabular}{llrr}
\hline & $\mathrm{H}$ & $\mathrm{C}$ & \multicolumn{1}{c}{ W } \\
\hline $\mathrm{H}$ & 0.00 & 7.65 & 2.86 \\
$\mathrm{C}$ & 7.65 & 0.00 & 14.30 \\
$\mathrm{~W}$ & 2.86 & 14.30 & 0.00
\end{tabular}


dimensionless $\kappa_{\mathrm{H}}{ }^{*}$ was previously obtained as $\kappa_{\mathrm{H}}{ }^{*}=\kappa_{\mathrm{H}} \nu_{\mathrm{p}} / k_{\mathrm{B}} T=$ 5.0 (with $\nu_{\mathrm{p}}$ the particle volume) for the same particle density. ${ }^{14}$

The FH parameters of Table 1 have been used in all simulations for the lipid-water system discussed in the results section. However, these parameters relate to the usual situation where the average of the total field is conveniently scaled to unity (via multiplication by an appropriate particle volume). In the projection algorithm, density fields are obtained from a particle-wise assignment of particle fractions to neighbouring grid points (summing up to one), followed by an additional point-wise multiplication of the density fields by a factor $1 /\left(l^{*}\right)^{3}$, which has the purpose of rendering the average total field $\bar{\phi}^{*}$ independent of the grid size $l$. In particular, defining $n_{x}=L_{x} / l, n_{y}=L_{y} / l$ and $n_{z}=L_{z} / l$, and writing $\mathbf{r}=(i, j, k)$ in units of the mesh size $l$, it is easy to show

$$
\begin{aligned}
\bar{\phi}^{*} & =\frac{1}{n_{x} \cdot n_{y} \cdot n_{z}} \sum_{K} \sum_{i, j, k=0}^{n_{x}, n_{y}, n_{z}} \frac{\phi_{\mathrm{K}}{ }^{*}(i, j, k)}{\left(l^{*}\right)^{3}} \\
& =\frac{N}{\left(l^{*} \cdot n_{x}\right)\left(l^{*} \cdot n_{y}\right)\left(l^{*} \cdot n_{z}\right)} \\
& =\frac{N}{L_{x}^{*} L_{y}{ }^{*} L_{z}{ }^{*}}=\frac{N}{V^{*}}=\phi_{0}{ }^{*}
\end{aligned}
$$

Since we are working with this non-unitary total field, we have to scale $\chi_{K K^{\prime}}$ by $1 / \phi_{0}^{*}$.

MD-SCF/MPCD is based on the Hamiltonian (7) and evolved using MD (velocity Verlet) and MPCD, which both conserve energy, so we simply conclude that our method is energy conserving. Focussing on numerical stability, the 'safe' upper bound for Martini CGMD, i.e. $\Delta t=30 \mathrm{fs},{ }^{28}$ was consistently used for all MD-SCF simulations (with an Andersen thermostat) that employed a Martini representation for their constituents. ${ }^{26}$ When applying MD-SCF/MPCD instead, this value seems a conservative but safe choice. However, since bonded interactions are not affected by the hybridisation, a DPD-like time step can be expected for the current lipid/water representation, which is adopted from DPD. As discussed by Groot and Warren, ${ }^{21}$ the choice of the dimensionless time step $\Delta t^{*}$ in DPD is determined by the amount of increased/artificial temperature $k_{\mathrm{B}} T^{*}-1$ that one is willing to accept: they define the safe $\Delta t^{*}$ as the one for which this increase is $2 \%$. For brevity, we refrain from showing all temperature evolutions in the Results section, but we only note here that our choice for $\Delta t^{*}=0.01$ was based on a quick levelling of the simulation temperature to a constant value that is consistently about $2 \%$ higher for this time step, for all lipid/water MD-SCF/MPCD simulations considered. We thus conclude that $\Delta t \approx 3 \mathrm{ps}$, which is estimated via the properties of water particles, is a safe choice: it is indeed in the same range as the earlier reported DPD value of 5 ps for this lipid model. ${ }^{44}$ However, as field projection is usually not performed every time step in MD-SCF/MPCD, e.g. for the solvent (lipid/solvent) systems, it is performed every tenth (fifth) VV step respectively, see discussion at the end of this section, selecting even larger $\Delta t^{*}$ may result in instability. We indeed find that $\Delta t^{*}=0.02$, the value employed for all DPD simulations in this study, results in a fluctuating, non- monotonic evolution of the simulation temperature when used in MD-SCF/MPCD for the lipid/solvent systems. However, when the field is projected every time step instead $\left(f_{\text {up }}=1\right)$, we recover proper behaviour with $k_{\mathrm{B}} T^{*}-1<2 \%$. Since $\delta t^{*} / \Delta t^{*}$ is necessarily an integer, we also tried $\Delta t^{*}=0.05$ (stable, $k_{\mathrm{B}} T^{*}-1 \approx 2 \%$ ) and $\Delta t^{*}=\delta t=0.1$ (unstable), quantifying the direct relation between the maximum safe time step and the field projection frequency. Also in the MD-SCF/MPCD simulations for pure solvents, where the field is updated every tenth time step for $\Delta t^{*}=0.01$, the temperature increase is less than $2 \%$ for all values of $\kappa_{\mathrm{H}}$ considered.

Finally, we have to select a proper grid size $l=l^{*} \cdot r_{\mathrm{c}}$ for the projection of particles to density fields (see the appendix for more details). Among other, this defines the resolution of the fields and forces in the hybrid model, which should be chosen wisely given the application in mind. First, we note that we are limited in the range of $l$-values at the lower end if we stick to the standard choice for the particle density $\phi_{0}{ }^{*}=3$ of DPD. The average number of particles per MPCD cell should be in the range $3-20,{ }^{46}$ so this is a proper choice also for MPCD. However, since the projection algorithm only considers neighbouring grid points, field gradients and thus the effective forces on the particles will become increasingly noisy for smaller $l^{*}$. For $l^{*}<1$ and $\phi_{0}{ }^{*}=3$, for instance, there will be less than three particles per grid cell on average, and it is easy to understand that very minute particle displacements will have a tremendous effect on the field gradients. As a compromise between resolution and smoothness, we choose $l=r_{\mathrm{c}}$ or $l^{*}=1$, meaning that 5 grid points should suffice to describe the lipid profile (as well as field gradients for the force calculation) for a lipid membrane, which has a thickness of roughly $5 \mathrm{~nm}$. Moreover, we use a Gaussian, evaluated on 27 points around the closest grid point to the particle, rather than the original trilinear interpolation on 8 points, for the reasons discussed before. From these considerations, it is clear that we do not expect to reproduce the membrane profile of DPD in full detail. However, we note that a CGMD lipid representation is better resolved in space, enabling a smaller choice of $l^{*}$. Moreover, smaller values of $l^{*}$ are possible for our DPD lipid representation, either by selecting a projection algorithm with additional grid points, which has the effect of further smoothing, or by choosing a larger particle density $\rho_{0}{ }^{*}$ (or, equivalent, a smaller $r_{\mathrm{c}}$ ). As the particle-to-field projection is the most compute intensive procedure, both will be at the expense of a reduced computational efficiency.

The frequency with which the projection algorithm is called, $f_{\text {up }}$, is an important parameter for the computational efficiency; an update frequency of $f_{\text {up }}=10$, i.e. in which the projection algorithm is called every tenth time step, was considered in the original MD-SCF model, and even larger values were found suited in specific cases. ${ }^{29}$ This value is determined by the optimum between efficiency and both the effective particle dynamics and field resolution, in particular how fast the density fields at the chosen resolution changes given the underlying particle dynamics. The value $f_{\text {up }}=10$ was considered a conservative choice, ${ }^{29}$ and we will use it as the initial value. Nevertheless, later on, we discuss the need for using smaller values. 


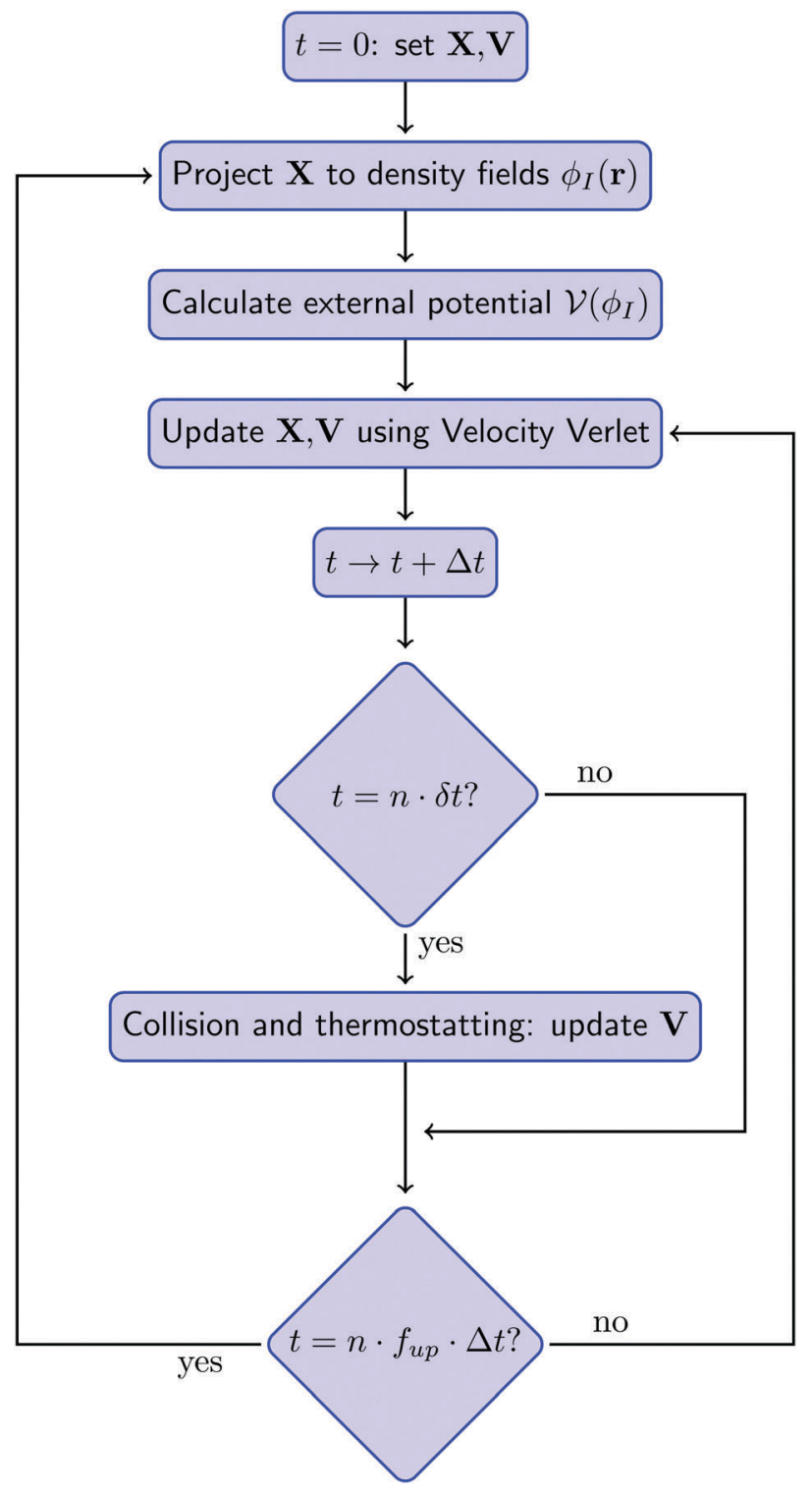

Flow diagram for the current method, with $\mathbf{X}$ and $\mathbf{V}$ denoting the position and velocities of all particles, $n$ an (arbitrary) integer, $\delta t$ the time step for collision and $f_{\text {up }}$ the update frequency for the field projection.

\section{Computational analysis}

When evaluating the performance of new methodology, one should be careful in separating the various aspects that contribute to efficiency. The first is the implementation into a computer algorithm, which enables a useful quantitative evaluation of efficiency in terms of the number of floating point operations (or the CPU time that they require) per time step in comparison to reference methods. The second are the conceptual steps made to generate a more efficient description of the underlying physics, here via (partial) coarsening and by accounting for the long-range hydrodynamic interactions.

The algorithmic advantage of hybrid MD-SCF/MPCD over pure-particle reference methods like (CG)MD and DPD, is the replacement of the force calculation for particle pairs by one that stems from particles interacting with chemical potential fields, after particle-to-field projection, which reduces the costs of this step and enables more efficient parallelisation. ${ }^{27}$ Nevertheless, the key advantage is the alternative physical description: since we replace the 'hard' Lennard-Jones interactions in (CG)MD by 'soft' mean-field interactions in MD-SCF/MPCD, we may replace the usual time step $\Delta t \sim$ fs that is required for stable integration in (CG)MD by the $\Delta t \sim$ ps time step of methods that consider soft-core interactions, like DPD. As a consequence, the sampling in the time domain is enhanced by several orders of magnitudes, unless bonded interactions dictate otherwise or the reference methods is already based on soft-core potentials. Since MD-SCF/MPCD inherits this property from MD-SCF, we refer to the literature for a detailed analysis. ${ }^{29}$ Furthermore, coupling MD-SCF to MPCD substantially accelerates the self- and re-organisation kinetics, see Results and discussion section, by restoring the hydrodynamic contribution to such processes.

We simulated a lipid/water system in two different volumes, $V_{1}=22^{3} l^{3}$ and $V_{2}=44^{3} l^{3}$, containing $N_{1}=31944$ particles (764 lipids) and $N_{2}=255552$ particles (3058 lipids), respectively. The number of lipids agrees with a flat membrane that spans the volume along two Cartesian coordinates. Table 2 shows timing results for algorithms that only differ in the implementation of the force update, the thermostat and/or the collision step. The depicted timings for a single time step $t_{\mathrm{s}}(V)$ (in CPU seconds) were obtained by averaging over a total of $10^{4}$ time steps for each simulation. We find that MD-SCF/MPCD is roughly a factor of two faster than DPD: 1.7 for $V_{1}$ and 2.3 for $V_{2}$. Since $N_{2}=8 N_{1}$, perfect scaling relates to a scaling factor $\zeta=t_{\mathrm{s}}\left(V_{2}\right) / t_{\mathrm{s}}\left(V_{1}\right)=8$, if we disregard the costs of the intramolecular forces calculation. Apparently, updating neighbour lists is demanding, as we find $\zeta=10.86$ for DPD, while $\zeta=8.00$ for both other methods. The costs of the MPCD collision step are modest ( $\approx 1 \%$ of the total). A comparison of MD-SCF/MPCD for $f_{\text {up }}=5$ or 10 shows that considerable computational gain can be obtained by performing fewer updates of the projection algorithm.

\section{Results and discussion}

\section{Pure solvent systems}

The hydrodynamic properties of a fluid simulated by MPCD/ SRD were considered in detail. ${ }^{47}$ This study identified two

Table 2 Time (averaged over $10^{4}$ time steps) in CPU seconds required for performing one (time) step with each of the three considered methods/ algorithms. Results were obtained using very similar serial codes, on a 2.8 GHz Intel Core i5 node with (shared) 8 GB 1333 MHz DDR3 memory

\begin{tabular}{lll}
\hline & Volume $V\left(\right.$ in $\left.l^{3}\right)$ & $t_{\mathrm{s}}(V)($ in s $)$ \\
\hline DPD & $22^{3}$ & 0.2261 \\
DPD & $44^{3}$ & 2.4561 \\
MD-SCF, Andersen & $22^{3}$ & 0.1311 \\
MD-SCF, Andersen & $44^{3}$ & 1.0473 \\
MD-SCF/MPCD, $f_{\text {up }}=5$ & $22^{3}$ & 0.1321 \\
MD-SCF/MPCD, $f_{\text {up }}=5$ & $44^{3}$ & 1.0530 \\
MD-SCF/MPCD,$f_{\text {up }}=10$ & $44^{3}$ & 0.7206
\end{tabular}


regimes, a gas-like and fluid-like, quantified by the Schmidt number $\mathrm{Sc}=\nu / D$, i.e. the ratio between viscous (kinematic viscosity $\nu$ ) and diffusive (diffusion coefficient $D$ ) transport coefficients. Moreover, it was found that the combined value of $\lambda^{*}$, the scaled mean free path, and $\alpha$, the rotation angle in the collision step, regulates this character. By considering the predicted Schmidt number $\mathrm{Sc}_{\mathrm{p}}$, i.e. using the $\nu$ and $D$ estimated from simulations, a fluid-like collective regime with $\mathrm{Sc}_{\mathrm{p}} \gg 1$ was identified for large $\alpha$ and small $\lambda^{*}$, and for small $\alpha$ and large $\lambda^{*}$ a gas-like particle regime with $\mathrm{Sc}_{\mathrm{p}} \leq 1$. The microscopic motions underlying the dynamical behaviour of the fluid were analysed via the normalised discrete velocity autocorrelation function (VACF) for $\lambda^{*}=0.1$ (collective regime) and 1.0 (particle regime), i.e.

$$
C_{v}(t)=\frac{\left\langle\mathbf{v}_{i}(t) \cdot \mathbf{v}_{i}(0)\right\rangle}{\left\langle\mathbf{v}_{i}(0) \cdot \mathbf{v}_{i}(0)\right\rangle},
$$

where $t=n \delta t^{*}$ is the discrete time relative to the starting time $n_{0} \delta t^{*}$ at the origin, and $\langle\cdot\rangle$ denotes an ensemble average over all particles and $n_{0}$.

To test our implementation, we calculated the VACFs for both $\lambda^{*}$ for the same parameter set (with angle of rotation $\alpha=130^{\circ}$ ) of the earlier study, ${ }^{47}$ see Fig. 1 . Since this system is ideal and the collision rules (14) conserve momentum and kinetic energy exactly for a fixed angle of rotation $\alpha$, a thermostat is not needed. All VACFs in this section were obtained by simple averaging over the last $4.9 \times 10^{4}$ consecutive time steps and over 10 independent simulations, each of which is started with a different noise seed. We note that especially the long time VACF behaviour is sensitive to the sampling statistics. From a comparison of Fig. 1 to Fig. 3 in ref. 47, we find that we reproduce the older results in this time regime.

Another important test is to analyse self-diffusion. Diffusion coefficients can be derived from the Green-Kubo formalism, but Ripoll et al mention that a linear fit of the mean-square displacement (MSD) for long times provides equivalent results. ${ }^{47}$ Fitting the MSD for our simulated systems, we find $D^{*}=0.64$ for $\lambda^{*}=1.0$ and $D^{*}=0.080$ for $\lambda^{*}=0.1$. Explicit values were not reported before, but we may compare the relative deviation $\Delta D=\left(D_{\text {sim }}-D_{0}\right) / D_{0}$, with $D_{0}$ an analytical prediction obtained using the Brownian approximation for the VACF in the Green-Kubo relation. ${ }^{47}$ We find $\Delta D=0$ for $\lambda^{*}=1.0$ and $\Delta D=0.25$ for $\lambda^{*}=0.1$, which is fully consistent with known results for the same parameter set, shown in Fig. 7 of the earlier study. ${ }^{47}$

Turning back to the VACF, for $\lambda^{*}=1.0$ and short times it closely follows the exponential decay that was predicted for a dense gas, in agreement with the system being in the particle regime. For $\lambda^{*}=0.1$, however, the behaviour of the VACF at shorter times deviates considerably from a gas-like exponential decay, signalling that cooperative effects play a role in slowing down the loss of velocity correlations over time. However, the VACF does not exhibit the typical negative region associated with backscattering or collective relaxation, i.e. transient caging imposed by neighbouring particles. At longer times, both VACFs decay algebraically as $t^{-3 / 2},{ }^{47}$ in agreement with the

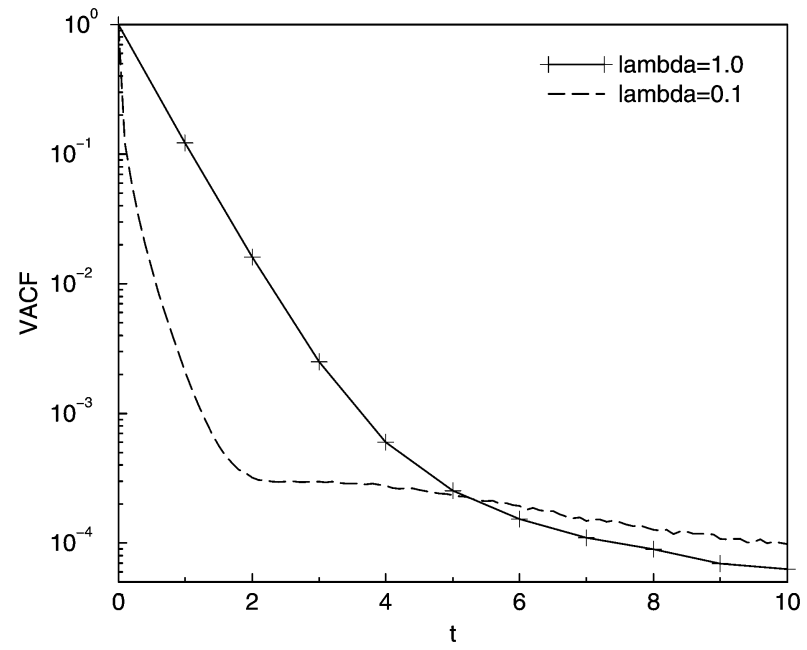

Fig. 1 The normalised VACF versus dimensionless time $t^{*}$ (half log plot) for two values of the scaled mean free path: $\lambda^{*}=0.1$ (collective regime, dashed line) and $\lambda^{*}=1.0$ (particle regime, solid line). For both $\lambda^{*}$, all other parameters are the same. In particular, we selected the same MPCD and volume parameters as in Fig. 3 in ref. 47 to test our implementation: cell size $a_{M P C D}{ }^{*}=1.0$, density $\rho_{0}{ }^{*}=5, \alpha=130^{\circ}$ and $L_{x}{ }^{*}=L_{y}{ }^{*}=L_{z}{ }^{*}=20$. This choice for the cell size implies that $\delta t^{*}=\lambda^{*}$. The shown graphs were obtained by averaging over the last $4.9 \times 10^{4}$ consecutive time steps and over 10 independent simulations, each of which is started with a different noise seed.

theoretical universal scaling relation for fluids in thermal equilibrium, ${ }^{48}$ see Fig. 2.

Backscattering or a negative region in the VACF is also absent for DPD simulations with only excluded volume interactions, ${ }^{45}$ whereas it was already observed in MD simulations of liquid water by Rahman and Stillinger, ${ }^{49}$ where non-bonded interactions are represented by Lennard-Jones potentials. The distinct oscillatory behaviour of the VACF of liquid water around the origin in the latter study, compared to simpler liquids that only carry one negative minimum, was attributed to hydrogen bonding, which results in a greater structural rigidity. ${ }^{49}$ We note that the particulars of the monotonic VACFs in DPD depend on numerical parameters, e.g. the chosen time step, and can be tuned by the choice of the friction coefficient $\gamma$. For the usual DPD value $\gamma=4.5$, the VACF is visually very similar to the one obtained for MPCD and $\lambda^{*}=0.1 .^{45}$

The effect of caging on the short-time transport properties is clearly not captured by the coarse treatments like MPCD and DPD, which lack the interactions that are responsible for this effect. One could, however, wonder what role compressibility plays in this phenomenon. Although the standard MPCD/SRD method does not impose incompressibility in any way, by design, the deviations of the solvent field $\phi_{\mathrm{W}}{ }^{*}(\mathbf{r})$ (obtained using the projection algorithm) from the reference value $\phi_{0}{ }^{*}$ are limited at all times in practice. We may, using the same notation as before, monitor the time-dependent variance $\Omega(t)$ of the solvent field, i.e.

$$
\Omega(t)=\sum_{i, j, k=0}^{n_{x}, n_{y}, n_{z}}\left(\phi_{\mathrm{W}}{ }^{*}(i, j, k, t)-\phi_{0}{ }^{*}\right)^{2}
$$



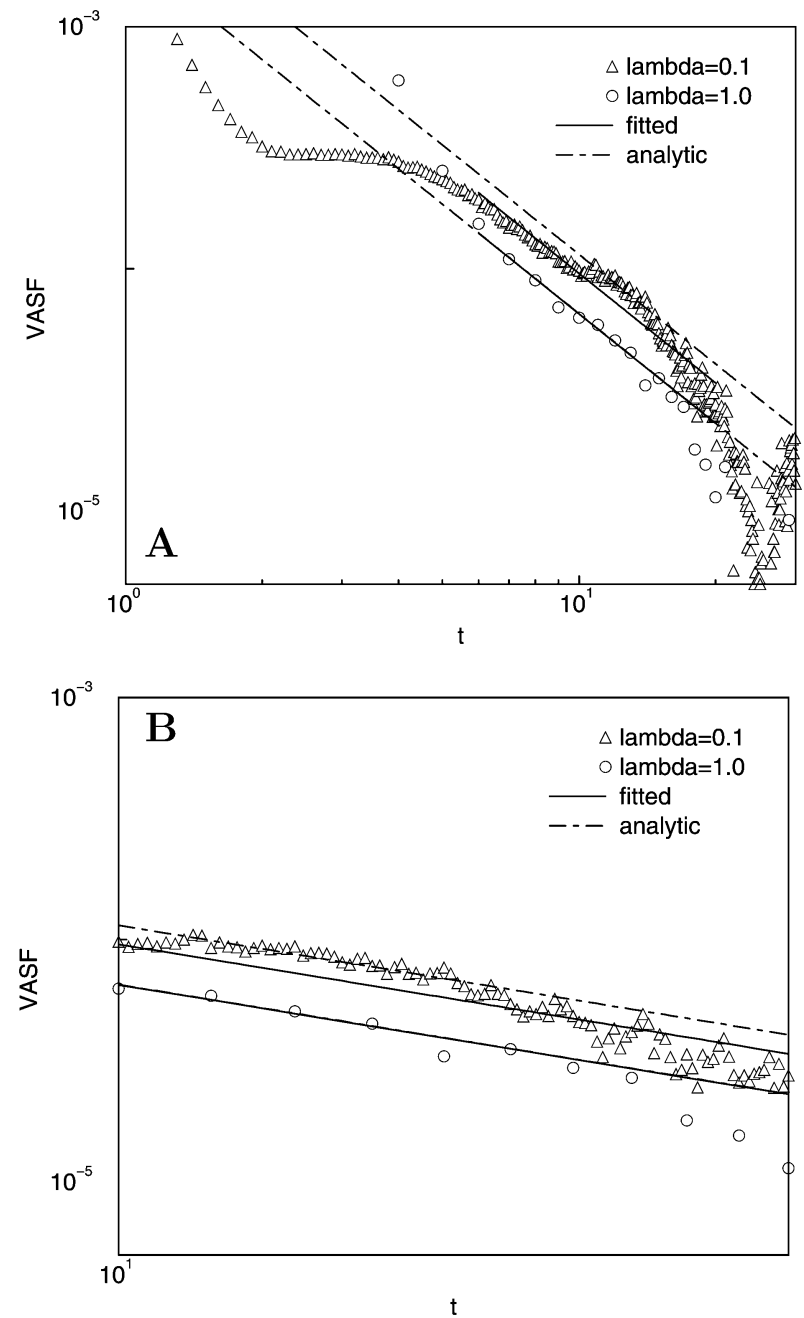

Fig. 2 Log-log plot of the same data as in Fig. 3, for $\lambda^{*}=0.1$ (triangles) and $\lambda^{\star}=1.0$ (circles), including the long-time tails. A: full time range, and B: zoom of tails. Lines relate to the predicted algebraic $\sim t^{-3 / 2}$ decay of the VACF, with an amplitude $a_{0}$ that is either obtained from the best fit to data points in a selected range ( $t^{\star} \in[6,20]$, solid line) or explicitly derived from modecoupling theory as $a_{0}=((d-1) / d \rho) \times(4 \pi(D+\nu))^{-d / 2}$ (dashed-dot line), see also Fig. 4 and discussion in ref. 47. To evaluate the latter expression, diffusion coefficients $D$ extracted from simulations were used and the kinematic viscosity $\nu$ was estimated from Fig. 1 and 2 in ref. 47 and the relation $\mathrm{SC}=\nu / D$, giving rise to $\nu^{\star}=0.80$ for $D^{\star}=0.080\left(\lambda^{*}=0.1, \mathrm{Sc} \approx 10\right)$ and $\nu^{\star}=0.64$ for $D^{\star}=0.64\left(\lambda^{\star}=1.0, \mathrm{Sc} \approx 1\right)$, inserting $d=3$ and $\rho^{\star}=\rho_{0}{ }^{*}=5$. Like in the previous study, ${ }^{47}$ we find that the predicted amplitude is exact within error bounds for $\lambda^{*}=1.0$ and that it is about $10 \%$ larger than the fitted one for $\lambda^{\star}=0.1$. Nevertheless, the zoom shows that the predicted decay (dashed-dot line) is reproduced for $\lambda^{*}=0.1$ at late times, i.e. next to the time range $\left(t^{*} \geq 20\right)$ where finite size effects start to dominate. ${ }^{47}$ Since we are interested in analysing how compressibility modulates the VACF, we will use both functions as a reference in the remainder.

to analyse the compressibility. Since MPCD solvent behaves as an ideal gas, the discrete probability $P\left(n_{\mathrm{c}}\right)$ for finding $n_{\mathrm{c}}$ particles per lattice cell is theoretically given by a Poisson distribution

$$
P\left(n_{\mathrm{c}}\right)=\frac{\left\langle n_{\mathrm{c}}\right\rangle^{n_{\mathrm{c}}} \mathrm{e}^{-\left\langle n_{\mathrm{c}}\right\rangle}}{n_{\mathrm{c}} !},
$$

with $n_{\mathrm{c}}$ the number of particles per cell and $\left\langle n_{\mathrm{c}}\right\rangle=n_{\mathrm{s}}=\phi_{0}{ }^{*}$ their average value. As a consequence of the variance per lattice cell being $\phi_{0}{ }^{*}$ in the steady state, we find that $\Omega(t)=\Omega_{\mathrm{st}}=V^{*} \phi_{0}{ }^{*}$.

Analysing $\Omega(t)$ from the simulations for $V^{*}=4000$ and $\phi_{0}{ }^{*}=5$, the first observation is that $\Omega(t)$ quickly reaches a plateau value $\bar{\Omega}$ that does not depend on $\lambda^{*}$, consistent with it being an equilibrium property. However, $\bar{\Omega}$ is lower than the theoretical value $\Omega_{\text {st }}=40000$, namely $\bar{\Omega}=2606$ with standard deviation $\sigma_{\Omega}=99$ for $\lambda^{*}=1$ and $\bar{\Omega}=2607$ and $\sigma_{\Omega}=103$ for $\lambda^{*}=0.1$. This deviation can be understood in terms of smoothing due to the particle-to-field projection, which assigns particle fractions instead of particles to lattice positions. As a result, the number of particles per cells $N_{\mathrm{p}}$ is usually a fractional number and thus the probability $P\left(N_{\mathrm{p}}\right)$ is a continuous instead of a discrete function. In particular, if we enforce discrete particle assignment, by projecting each particle fully to the closest lattice position, both the Poisson distribution and the theoretical value $\bar{\Omega}=V^{*} \phi_{0}{ }^{*}$ are recovered. From simulations with the original trilinear and the new Gaussian projection schemes, for different values of $\phi_{0}{ }^{*}$ and $V^{*}$, we find the relation $\bar{\Omega}=s V^{*} \phi_{0}{ }^{*}$, containing an additional constant $s$ which is fitted as $0.295(\approx 3 / 10)$ for trilinear and 0.064 $(\approx 1 / 15)$ for Gaussian interpolation. We note that the particle evolutions, and thus the equations of state, do not depend on this projection: they are the same in all considered cases. It just show that the variance of the projected solvent field depends on the projection algorithm. As expected, the smoothing is most significant for the Gaussian projection algorithm.

These numerically obtained values for an ideal gas can be used for a comparison to the new hybrid MD-SCF/MPCD method, which includes a field-based compressibility term. For the same $\alpha$ and $\lambda^{*}=0.1$, we replace the ballistic motion of MPCD by the velocity Verlet (VV) scheme (12) with a non-ideal force $\mathbf{f}_{i}(\mathbf{r})=-\kappa_{\mathrm{H}} \nabla_{\mathbf{r}} \phi_{\mathrm{W}}(\mathbf{r})$ for all particles and a time step $\Delta t$. The collision step, carrying a time step $\delta t$, is the same as in (14). Note that we recover the original MPCD scheme when the compressibility term is omitted $\left(\kappa_{\mathrm{H}}=0\right)$. Due to the excluded volume interactions, the kinetic energy is not necessarily conserved, and we apply the MBS thermostat during the collision step to maintain a constant simulation temperature. The time increment for the VV-scheme is $\Delta t^{*}=0.01$. For $\rho_{0}{ }^{*}=5$, a value of $\kappa_{\mathrm{H}}{ }^{*}=3$ was determined earlier, ${ }^{14}$ which sets a realistic range for the Helfand parameter. The field $\phi_{\mathrm{W}}$ is updated with $f_{\mathrm{up}}=10$, i.e. at every collision step.

Fig. 3 shows the short-time VACF for these systems for different values of the Helfand compressibility parameter $\kappa_{\mathrm{H}}$ in this range. We find that adding excluded volume interactions gives rise to effective caging, as can be concluded from the presence of negative regions in the VACF. The origin of this phenomenon lies in the understanding that particles experience a force that drives them towards a situation where the variance of $\phi_{\mathrm{W}}(\mathbf{r})$, the field derived from the instantaneous particle positions, decreases in between collision steps, i.e. large fluctuation of the solvent field are suppressed. In particular, $\bar{\Omega}\left(\kappa_{\mathrm{H}^{*}}\right)$ decreases for increasing $\kappa_{\mathrm{H}}{ }^{*}$, see Fig. 4 , as one may expect, indicating also that sound waves are not an issue. Since these forces oppose the build-up of regions of too low and high 


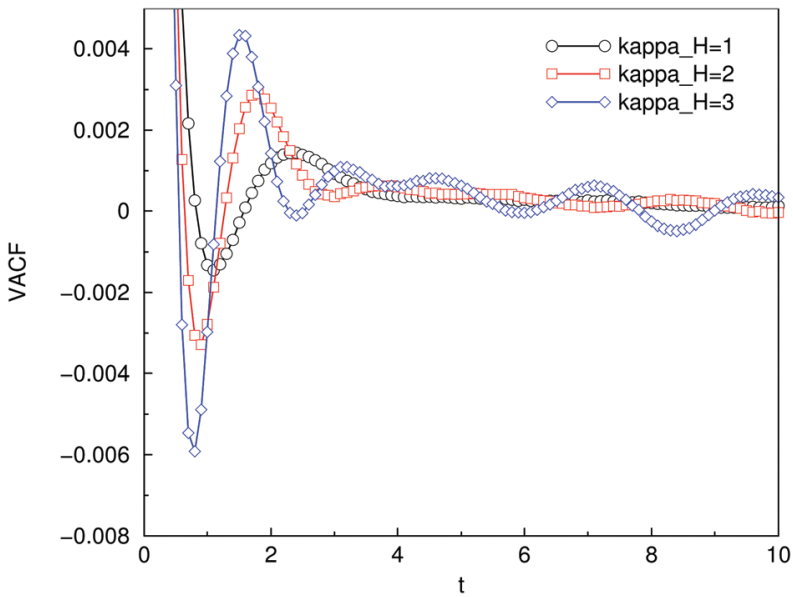

Fig. 3 The normalised VACF versus dimensionless time for a noncohesively interacting gas $(\chi=0)$ in the new MD-SCF/MPCD approach, where compressibility is penalised via the Helfand compressibility term in the non-ideal free energy. For $\kappa_{H^{*}}{ }^{*}=0$, standard MPCD is recovered. The graphs show results for increasing compressibility parameter: $\kappa_{H^{*}}=1$ (black, circles), $\kappa_{\mathrm{H}}{ }^{*}=2$ (red, squares) and $\kappa_{\mathrm{H}}{ }^{*}=3$ (blue, diamonds). The latter value relates the compressibility of the system to that of liquid water at room temperature. We used the same system parameters as before and a MPCD time step $\delta t^{\star}=\lambda^{\star}=0.1$ (collective regime). The time step for the VV scheme (12) is $\Delta t^{\star}=0.01$. The shown graphs were obtained by averaging over the last $4.9 \times 10^{4}$ consecutive time steps and over 10 independent simulations, each of which is started with a different noise seed.

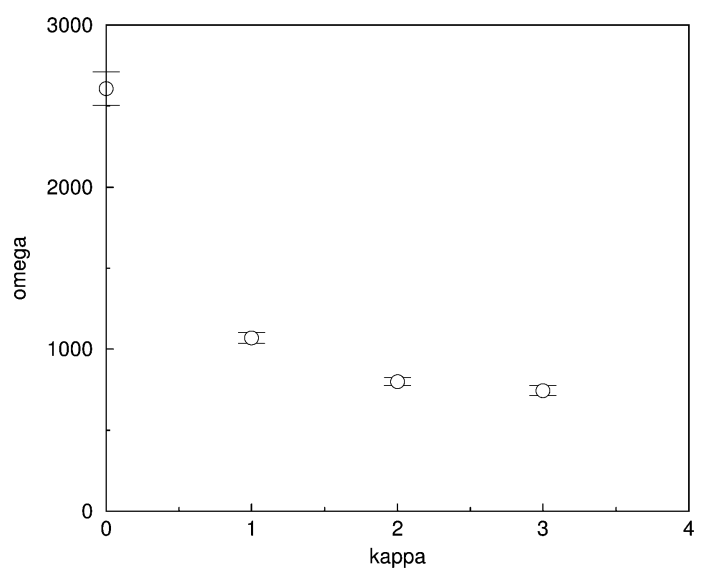

Fig. 4 The mean value $\bar{\Omega}$ of $\Omega(t)$ in (20) for different values of $\kappa_{\mathrm{H}^{*}}$, as determined over the whole trajectory. In the simulations, $\Omega(t)$ very quickly levels off to a constant value around which it fluctuates. Bars reflect the standard deviation $\sigma_{\Omega}$ of these fluctuations. From the observation that $\bar{\Omega}$ monotonically decreases for increasing $\kappa_{\mathrm{H}^{*}}$, we conclude that sound waves play no role when compressibility is accounted for. In particular, unphysical sound waves are absent for $\kappa_{\mathrm{H}}{ }^{*}=0$ (standard MPCD).

particle densities, particles experience an effective caging by their surroundings.

Our treatment deviates in this aspect from the original hybrid approach of Malevanets and Kapral, ${ }^{42}$ which neglects all solvent-solvent interactions in the MD propagation scheme between collision steps. Their theoretical analysis of MD/SRD as a numerical solver for the Navier-Stokes equations starts from the ballistic streaming step for solvent particles, and it is not directly apparent if and how including direct solventsolvent interactions in the MD updates, like the ones advocated here, affect this hydrodynamic description. Padding and Louis ${ }^{50}$ did introduce additional terms in the inner MD loop for a pure solvent system, but they restrict themselves to external forces (due to gravity, fixed or moving boundary conditions) on solvent particles and disregard any direct force between solvent particle pairs. Disregarding them, however, seems to be purely a matter of convenience and not a methodological constraint. ${ }^{50}$ After all, representing solvent-solvent interaction only by collisions is favoured in terms of computational efficiency and analytical tractability.

Instead of a formal justification, we may study the longertime scaling for the simulated systems with $\kappa_{\mathrm{H}}{ }^{*}>0$. In Fig. 5, we have plotted the absolute value of VACF versus $t^{*}$ for the three considered values of $\kappa_{\mathrm{H}}{ }^{*}$ on a $\log -\log$ scale. The algebraic fitting functions shown in Fig. 2, i.e. $a_{0} t^{-3 / 2}$ with $a_{0}$ determined either by regression or from mode-coupling theory, both for $\lambda^{*}=0.1$, are added to enable a direct comparison to the results of the original MPCD scheme. We note that, as before, the behaviour starting from $t^{*} \approx 15-20$ can be attributed to the finite system size and should be disregarded in this analysis. ${ }^{47}$ Although the trends are certainly less well-defined than for $\kappa_{\mathrm{H}}{ }^{*}=0$, the known algebraic $t^{-3 / 2}$ decay is reproduced reasonably well by the simulations results for $\kappa_{\mathrm{H}}{ }^{*} \neq 0$, most clearly for $\kappa_{\mathrm{H}}{ }^{*}=1$ and 2 . This analysis suggests that the hydrodynamics on a larger scale is not significantly affected by the introduction of the (weak) particle pair interactions, but a formal derivation is left for the future. Also intuitively one could expect this finding, since the original collision scheme is conserved. The diffusion coefficient, obtained from the MSD for these simulations,

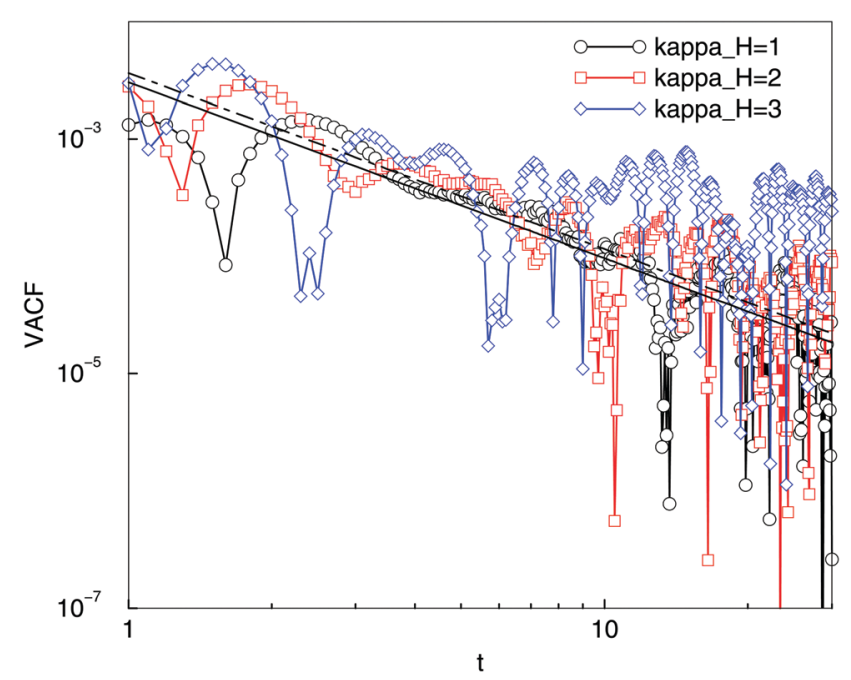

Fig. 5 Log-log plot of the absolute value of the normalised VACF shown in Fig. 3, for $\kappa_{\mathrm{H}}{ }^{*}=1$ (black, circles), $\kappa_{\mathrm{H}}{ }^{*}=2$ (red, squares) and the realistic value $\kappa_{H^{*}}=3$ (blue, diamonds), zooming in on the long time tails behaviour. Taking the absolute value is required because of the negative regions in the VACF. The algebraic decay $a_{0} t^{-3 / 2}$ of standard MPCD is added for comparison, see Fig. 2. Solid line: with numerically fitted $a_{0}$; dashed-dotted line: for $a_{0}$ calculated using mode-coupling theory. 
is $D^{*}=0.079$ for all $\kappa_{\mathrm{H}}^{*} \neq 0$ (compare to $D^{*}=0.080$ for standard MPCD with $\kappa_{\mathrm{H}}^{*}=0$ ). This finding confirms that self-diffusion is hardly affected by the additional control of the compressibility.

In addition to $f_{\text {up }}=10$, where the field is projected every tenth VV step or every collision step, we have performed simulations for $\kappa_{\mathrm{H}}{ }^{*}=3$ and field updates every fifth VV step, i.e. $f_{\text {up }}=5$, the standard value in the next sections, and even every VV step, i.e. $f_{\text {up }}=1$ (fully synchronised). The original model of Milano and Kawakatsu generally employed much fewer updates, i.e. $f_{\text {up }} \sim 10-100,{ }^{29}$ based on the observation that projected fields are usually only slowly fluctuating. Although fewer updates are certainly computationally attractive, it also signals that the underlying particle dynamics in the MD-SCF model is rather slow. Selecting $f_{\text {up }}=1$ can be seen as a test for the consistency between the field projection and the underlying particle dynamics in the current treatment.

Fig. 6 compares the short time and long-time behaviour of the VACFs for $f_{\text {up }}=10, f_{\text {up }}=5$ and $f_{\text {up }}=1$. Again, we may disregard the very long-time tails, where the restricted system size becomes an issue. It is clear that also the VACF for the fully synchronised case contains a negative region and that the graph oscillates, albeit that the amplitude of these oscillations is significantly smaller than for $f_{\text {up }}=10$. The latter can be understood in terms of the temporarily static nature of the concentration field for $f_{\text {up }}>1$. Since the particle dynamics is driven by this field, updating the field less frequently $\left(f_{\text {up }}=10\right)$ is equivalent to increasing the equilibration time towards an optimal particle configuration given that (static) field, which clearly enhances the caging. The position of the local VACF minima, however, does not notably depend on $f_{\text {up }}$, and filtering these VACFs, to damp the oscillations, shows that they feature equivalent long-time behaviour. The algebraic long-time decay $\left(\sim t^{-3 / 2}\right)$ is already quite apparent for $f_{\text {up }}=1$.

\section{Lipid-solvent systems}

Next, we consider systems containing $n_{1} \mathrm{H}_{3}\left(\mathrm{C}_{4}\right)_{2}$ lipids and $n_{\mathrm{w}}$ single-bead $\mathrm{W}$ solvent in a volume $V^{*}=L_{x}{ }^{*} L_{y}{ }^{*} L_{z}{ }^{*}$ of varying size, subject to periodic boundary conditions. The grid size $l$ for the particle-to-field projection is usually set to $l=r_{\mathrm{c}}\left(l^{*}=1\right)$, but we will also consider other values. As mentioned before, we renormalise the overall particle density, which varies with $l$, to $\phi_{0}{ }^{*}$ after every particle-to-field projection. Here, we consider the standard value $\phi_{0}{ }^{*}=3$ for DPD. The total number of particles $N$ is constant and given as $N=n_{\mathrm{w}}+11 n_{1}=3 V^{*}$. The $\mathrm{FH}$ parameters are fixed in all simulations, i.e. $\chi_{\mathrm{HC}}=7.65 / 3$, $\chi_{\mathrm{HW}}=2.86 / 3$ and $\chi_{\mathrm{CW}}=14.30 / 3$ (see the Simulation setup subsection). The average area per lipid $\mathcal{A}$ for a tensionless membrane was previously determined as $\mathcal{A}_{0}=1.26$ (in units of $\left.r_{\mathrm{c}}{ }^{2}\right) .{ }^{44}$ We determined an equivalence between the compressibility parameter $\kappa_{\mathrm{H}}=5$ and the DPD $a_{i i}=25$ for a particle density $\phi_{0}{ }^{*}=3$, via the equation of state for the pressure, although we used $\kappa_{\mathrm{H}}=4.6$ before. ${ }^{14}$ Since this parameter controls the lipid spacing within a membrane, we will vary its value within this small range.

To test whether our set of thermodynamic parameters $\left\{\chi_{K K^{\prime}}, \kappa_{\mathrm{H}}\right\}$ provide a proper mapping between the hybrid MD-SCF/MPCD
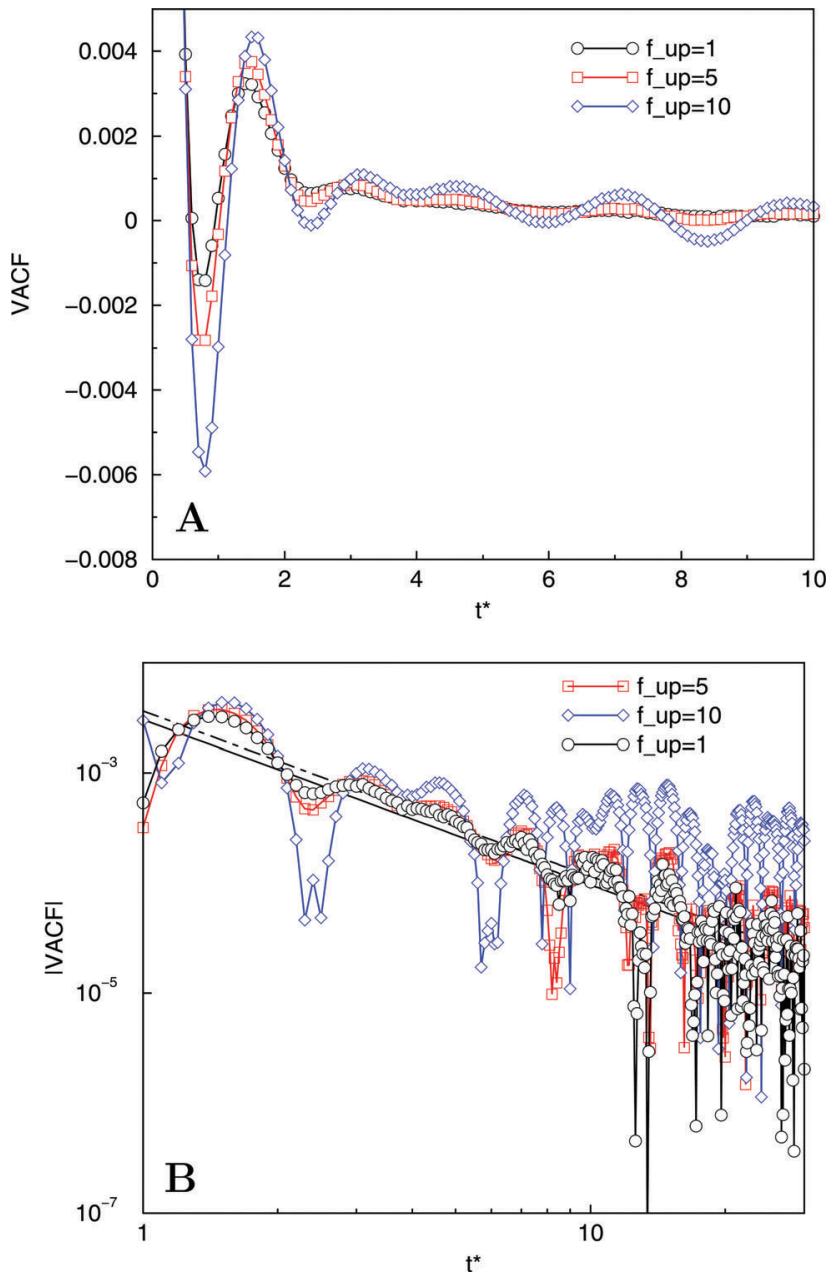

Fig. 6 Comparison of the normalised VACF for $f_{\text {up }}=10$ (blue, diamonds), $f_{\text {up }}=5$ (red, squares) and $f_{\text {up }}=1$ (black, circles) for $\kappa_{\mathrm{H}^{*}}{ }^{*}=3$. The time step for the VV scheme (12) is $\Delta t^{\star}=0.01$ and $\lambda^{\star}=\delta t^{\star}=0.1$ in the MPCD part. All other parameters are the same as in Fig. 3. A: the normalised velocity autocorrelation function versus dimensionless time. B: log-log plot of the long-time tails of the absolute value of the normalised velocity autocorrelation function versus dimensionless time. The algebraic decay $a_{0} t^{-3 / 2}$ of standard MPCD is added for comparison, see Fig. 2. Solid line: with numerically fitted $a_{0}$; dashed-dotted line: for $a_{0}$ calculated using modecoupling theory. The shown graphs were obtained by averaging over the last $4.9 \times 10^{4}$ consecutive time steps and over 10 independent simulations, each of which is started with a different noise seed.

and the reference DPD model, we initially distribute a wellchosen number of lipids, i.e. $n_{1}=2 L_{x} L_{y} / \mathcal{A}_{0}$, at random locations in the volume. By selecting $n_{1}$ consistent with a flat or planar tensionless DPD bilayer along two Cartesian directions, the sensitivity of the surface tension to the average area per lipid $\mathcal{A}^{44}$ in our $N V T$ ensemble is exploited. Spontaneous self-assembly in the hybrid model should give rise to a planar bilayer; if another (tensionless) structure is formed, either it is a metastable state or our parameters should be tuned. For reasons of efficiency, we avoid a field update at every time step, but instead select a small value $f_{\text {up }}=5$, assuming that the field does not significantly change between updates for this value. It was determined from test simulations for different $f_{\text {up }}$ and it is actually 
smaller than the usual minimal choice in $\mathrm{MD}-\mathrm{SCF},{ }^{29}$ in line with our expectation that the field evolution is accelerated by accounting for collisions. The cell size $a$ in the MPCD scheme is initially chosen as small as possible, $a^{*}=l^{*}=1$, to maximise the resolution of MPCD. We note that this choice relates to roughly 3 particles per collision cell, which is fairly small compared to the usual values of $5-15,{ }^{47}$ but within the range where MPCD is valid. ${ }^{46}$ The rotation angle $\alpha$ and collision time step $\delta t$ in MPCD are set to $\alpha=90^{\circ}$ and $\delta t^{*}=0.1$, which positions the dynamics in the solvent domain in the fluid-like regime. ${ }^{47}$ The time step used in the VV-scheme is $\Delta t^{*}=0.01$, with collisions (and thermostatting) thus taking place every 10 steps of the inner loop.

Consistency of the MD-SCF/MPCD parameters. We start with a small cubic volume $V^{*}\left(L_{x, y, z^{*}}=22\right)$ containing $n_{1}=764$ lipids and $n_{\mathrm{w}}=23540$ solvent particles. The reference DPD simulation, using parameters of Shillcock et al. ${ }^{44}$ shows that a planar membrane readily forms by breakup and coarsening of an initially interconnected structure. Close examination of the structural evolution, however, shows that the limiting factor is the diffusion and uptake of solvated lipid micelles (here: one micelle) that result from this breakup process. After approximately $10^{5}$ time steps, with $\Delta t^{*}=0.02$, this process is completed (see Fig. 7A and $\mathrm{A}^{\prime}$ ).

Next, we consider the same system using our new hybrid model, varying the compressibility parameter between $\kappa_{\mathrm{H}}=4.6$ (ref. 14) and $\kappa_{\mathrm{H}}=5.0$, the theoretically predicted value for this
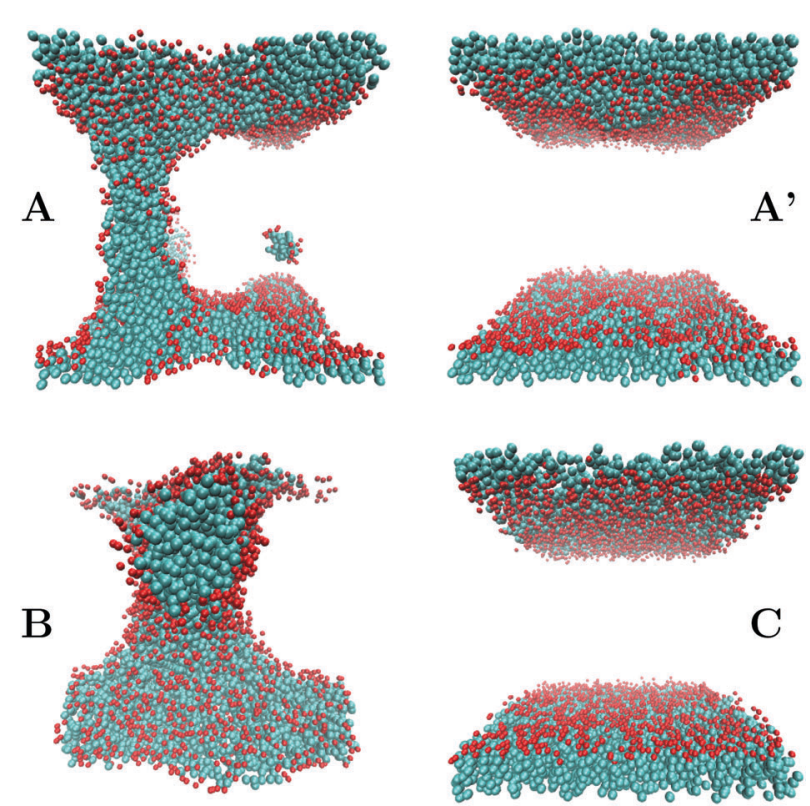

Fig. 7 Simulation snapshots after $20000(A)$ and $100000\left(A^{\prime}\right)$ time steps of DPD $\left(\Delta t^{*}=0.02\right)$. Just prior to the stage shown in the right hand side, a long-lived lipid micelle has merged with the bilayer. (B) Simulation snapshot after 10000 steps of the new hybrid method for $\kappa_{\mathrm{H}}=4.6$. (C) Simulation snapshot after 10000 steps of the new hybrid method for $\kappa_{\mathrm{H}}=5.0$. The mean-field free energy for the structures in the bottom row, obtained using $\Delta t^{*}=0.01$, has reached a constant value, suggesting that they are equilibrium structures. For clarity, only $\mathrm{H}_{3}\left(\mathrm{C}_{4}\right)_{2}$ lipids are shown, with red/blue representing $\mathrm{H} / \mathrm{C}$ particles. In all simulations, periodic boundary conditions were employed. density. When both the changes in the non-ideal free energy $W[\phi]$ and the lipid structure are negligible beyond a certain stage, we denote that structures as equilibrium. For $\kappa_{\mathrm{H}}=4.6$, lipids rapidly assemble from a mixed phase, via intermediate spherical and cylindrical micellar phases, into a stable spacefilling interconnected structure on a large scale, see Fig. 7B, while a planar lipid bilayer, aligned with two of the Cartesian axes, rapidly assembles for $\kappa_{\mathrm{H}}=5.0$ (Fig. 7C). Considering the structure for $\kappa_{\mathrm{H}}=4.6$, which is exemplary for a (meta)stable structures in the range $\kappa_{\mathrm{H}} \in\{4.7,4.8,4.9\}$, more carefully, we find that the lipid-water interface is reminiscent of a unit cell of a Schwarz minimal surface type $P$, i.e. a triply periodic surface with minimal surface area and vanishing mean curvature, albeit that it is slightly distorted. We note that continuing this simulation, up to a total of $2 \times 10^{5}$ time steps confirms that the structure shown in Fig. 7B is (meta)stable.

To obtain a better insight in the peculiarities of the simulation results, we quantify all structures in terms of geometrical and topological features of the lipid-water interface via a procedure that determines the four Minkowski functionals (MFs) for the total lipid field, $\phi_{\mathrm{l}}(\mathbf{r})=\phi_{\mathrm{H}}(\mathbf{r})+\phi_{\mathrm{C}}(\mathbf{r}){ }^{51,53}$ For the interconnected equilibrium structure shown in Fig. 7B, one of the MFs, the Euler characteristic $\chi_{\mathrm{MF}}$, is indeed -2 , which is the theoretical value for the unit cell of a P-surface. ${ }^{52,54}$ Other theoretical MF values for a P-surface unit cell are 0.5 (volume), 2.345 (surface area), and 0 (integral mean curvature). ${ }^{54}$ For the planar membrane, we find $\chi_{\mathrm{MF}}=0$ as a result of the periodic boundary conditions, which relate this structure to a vesicle, i.e. a spherical object with one internal cavity. ${ }^{51}$ Before analysing the geometrical MFs, we note that our procedure overestimates actual values for curved surfaces because of the two-fold discretisation, from particlesto-field and field-to-voxels, that is needed for our procedure, ${ }^{54}$ unlike the topological Euler characteristic, which has an integer value that is not affected by the discretisation procedure. We nevertheless consider our procedure sufficient for the current analysis. For more sensitive analysis, e.g. to properly analyse the geometrical properties of the bicontinuous connection region that forms upon fusion between two membranes, the calculation procedure could in principle be improved by using a finer grid to project particles directly to voxels instead of using field values on the fixed computational grid.

Comparison of the MFs that are related to the total surface area and integral mean curvature, along the pathway for the two values of $\kappa_{\mathrm{H}}$, shows that the characteristics of structure evolution overlap in the initial stages, see Fig. 8. Moreover, the decrease of the integral mean curvature to zero with time for $\kappa_{\mathrm{H}}=5$ confirms the completion of a planar bilayer around $10^{4}$ time steps.

We may alternatively view our membrane as a thin elastic sheet, and write a Helfrich effective surface Hamiltonian $\mathcal{H}$ for a symmetric bilayer membrane

$$
\mathcal{H}=\int_{A} \mathrm{~d} S\left[\sigma+2 \kappa_{\mathrm{c}} H^{2}+\bar{\kappa} K\right],
$$

with $\sigma$ the surface tension, $A$ the total surface area, $\kappa_{\mathrm{c}}$ and $\bar{\kappa}$ the bending modulus and Gaussian bending modulus, respectively, 

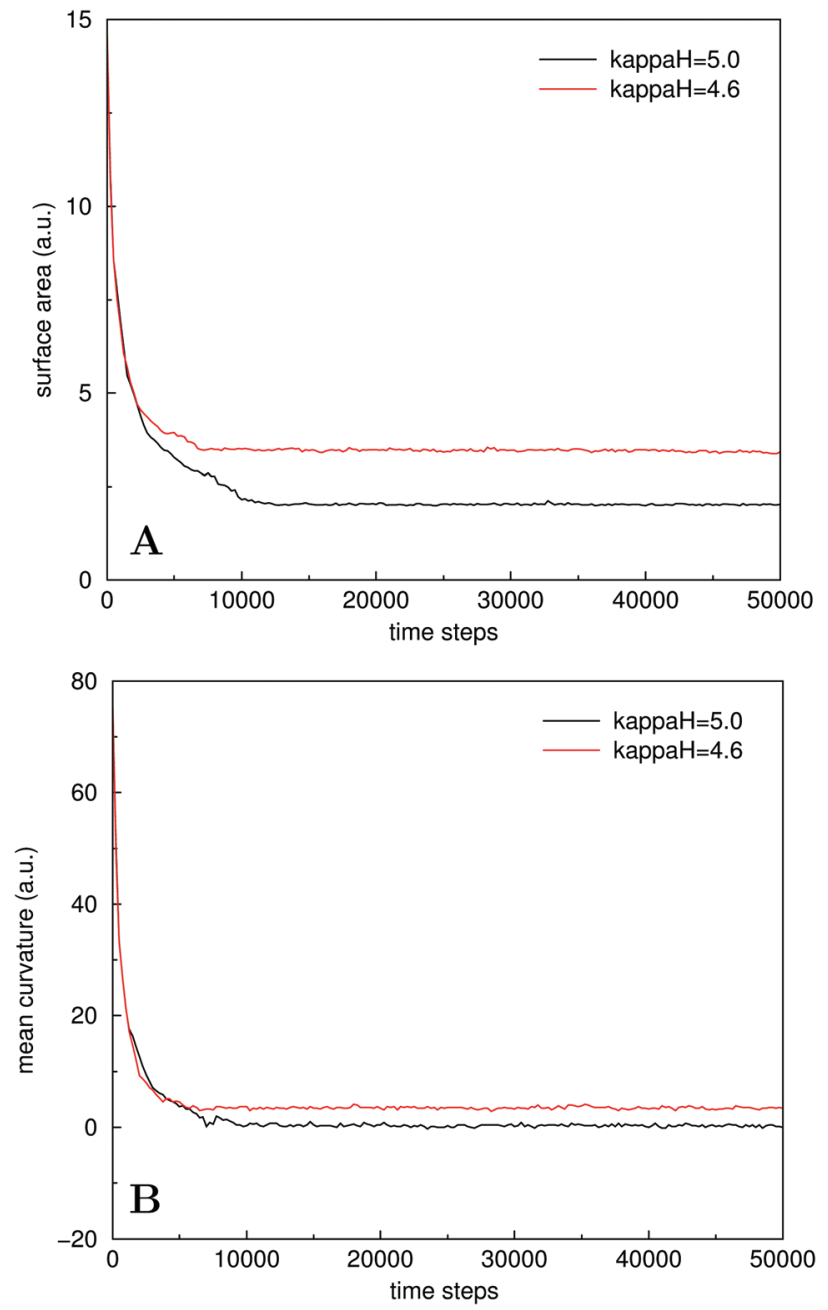

Fig. 8 Evolution of the Minkowski functionals related to the (total) surface area (A) and integral mean curvature (B), for structures along the pathways for $\kappa_{\mathrm{H}}=4.6$ and 5.0. The MFs were calculated and scaled using the procedure described in ref. 53. In short, they were calculated from the $22 \times 22 \times 22$ voxels in a binary (b/w) representation of the total lipid field that is obtained via a thresholding procedure (threshold 1.0). The total lipid field is the sum of field values for head and tails on the computational grid.

and $H=\left(c_{1}+c_{2}\right) / 2$ and $K=c_{1} \cdot c_{2}$ the local mean and Gaussian curvature, with $c_{1}$ and $c_{2}$ the local principal curvatures. Although a thin elastic sheet representation is not adequate for small lipid domains with high curvature, i.e. some of the simulated structures in this study, it is not uncommon to seek additional insight by employing Helfrich theory also for these cases. For the membrane considered here, the bending modulus was previously determined as $\kappa_{\mathrm{c}}=25 k_{\mathrm{B}} T$ and the number of lipids consistent with a tensionless flat membrane, ${ }^{60}$ although one could wonder if these values are also appropriate for MD-SCF/MPCD. The term $\bar{\kappa} K$ in (22) is usually omitted, as it is constant when the Euler characteristic does not change, via the Gauss-Bonnet theorem. Moreover, it vanishes for a structure with $K=0$, like a planar or cylindrical structure, if we neglect shape fluctuations that are unimportant for our analysis. The challenge here is that the Euler characteristic for a plane and a P-surface are different, and the Gaussian curvature for the P-surface is non-zero, meaning that the extra term should be added to compare the Helfrich energy for these two structures.

A planar membrane $(H=0)$ that is tensionless $\left(\sigma^{*}=0\right)$ for an average area per lipid $A>A_{0}$, as a result of an unmatched $\kappa_{\mathrm{H}}$, will experience a non-zero (positive) surface tension $\sigma$ when assembled from a number of lipids consistent with $\mathcal{A}_{0} \cdot{ }^{44}$ A mismatch of $\kappa_{\mathrm{H}}$ will therefore destabilise a planar membrane in favour of structure associated with lower surface tension, as long as the topology does not change. The number of accessible structures or states, however, is limited by the forced periodicity in all three dimensions, which rules out simple tilting to relieve surface tension, and will thus affect the barriers between accessible states. The quadratic term in (22) vanishes for a triply-periodic P-surface, which pairs a minimal surface area to a vanishing mean curvature. Fig. 8 shows that both MFs for $\kappa_{\mathrm{H}}=4.6$ are indeed quite small, particularly if we consider that the structure is curved and the voxel-based calculation procedure over-estimates these MF values. In combination with visual inspection, we therefore conclude that this interface bears many features of a triply-periodic P-surface.

The finding that the surface area for the P-like structure is nevertheless larger than for a planar membrane, see Fig. 8, is somewhat surprising, as it is a minimal surface structure. Although this can be seen in terms of an over-estimation of the surface area, it can also be understood in terms of periodicity constraints. In particular, the space-filling property is not satisfied for a planar membrane, which is periodic in only two dimensions.

Direct comparison of the Helfrich free energy for perfect planar and P-type structures is not straightforward, since it requires reliable information of the principal curvatures, surface tensions and $\bar{\kappa}$. Nevertheless, combining $K=-c_{1}{ }^{2}$ (for a perfect P-surface, $c_{2}=-c_{1}$ ) and the experimentally consistently found relation $\bar{\kappa} \approx-(0.8-0.9) \kappa_{\mathrm{c}}$, we may conclude that the surface tension for the triply-periodic structure is likely smaller than for its planar counterpart in the case of a $\kappa_{\mathrm{H}}$-mismatch, as one would expect for a structure that forms by spontaneous assembly. This indicates that the surface tension is indeed non-zero for $\kappa_{\mathrm{H}} \neq 5.0$.

For $\kappa_{\mathrm{H}}=4.6$, we do have direct access to the non-ideal free energy $W[\phi]$ for a pre-assembled (stable) planar membrane and the defected triply-periodic membrane of Fig. 7B. Close comparison (not shown) indicates that the difference is indeed very marginal, albeit that the planar membrane has the lowest non-ideal free energy. Apparently, the connections in the interconnected structure that always quickly forms upon quenching a lipid/solvent mixture cannot be broken for $\kappa_{\mathrm{H}}=4.6$, and coarsening proceeds by minimising the mean curvature and surface area, resulting in a (meta)stable P-like structure. The energy barrier separating the two states can only be surpassed for $\kappa_{\mathrm{H}}=5.0$.

Setting $\kappa_{\mathrm{H}}=5.0$, we first focus on the concentration profiles perpendicular to the planar membranes, as obtained from standard DPD and the hybrid MD-SCF/MPCD model for two values of the grid size $l$ used in the projection of particles to concentration fields, see Fig. 9. We note that all profiles were 

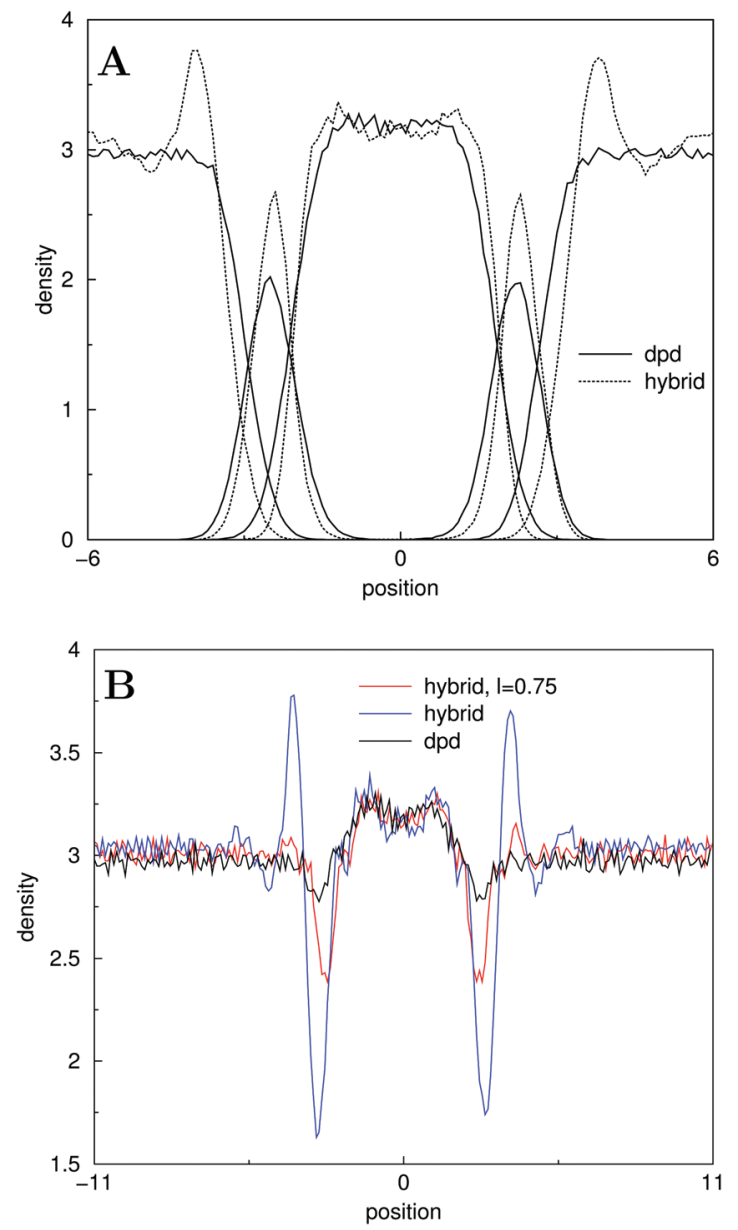

Fig. 9 (A) Comparison of the concentration profiles of a self-assembled membrane simulated using standard DPD (solid lines) with a self-assembled membrane for the new hybrid model, $l=1.0 r_{\mathrm{c}}$ and the cell size for the MPCD collision step $a=l$ (dashed lines). (B) The total concentration profile, obtained by summing the individual profiles, for the same two systems and a pre-assembled membrane with the new hybrid model $\left(l=0.75 r_{\mathrm{c}}\right.$ and $\left.a=2 l\right)$ discussed later on. The profiles for solvophilic head $(H)$, solvophobic tail (C) and solvent (W) particles were determined from binning instantaneous particle positions and averaging over a large number of simulation snapshots. For completeness, we note that a complete overlap of the MD-SCF/ MPCD and DPD profiles is not to be expected, as the methods are based on different degree of coarsening.

obtained directly from particle positions (by a binning procedure) and averaging over the last 160 snapshots; the reference DPD profiles were determined for the system shown in Fig. 7A and $\mathrm{A}^{\prime}$ by the same procedure. Comparing these profiles to the ones for the hybrid model, i.e. the system shown in Fig. 7C, we observe good agreement, albeit that the particles in the hybrid model are more localised - the profiles are sharper, see Fig. 9A - and that there is a solvent enrichment close to the membrane, compared to the bulk value. Although these deviations seem irrelevant, it makes sense to trace their origin.

It is useful to compare the profiles for the total concentration, see Fig. 9B, obtained by summing over the profiles for particle types. For DPD, we observe the characteristic small dip next to the membrane and a corresponding rise of the total particle concentration within the membrane. For the hybrid MD-SCF/MPCD method, the general features of the total concentration profile are comparable, but the rise/dip at the lipid-water interface are more pronounced, despite the presence of a compressibility term in the non-ideal free energy that penalises such localised deviations.

Two remarks can be made. First, these concentration profiles are directly derived from particle coordinates, while MD-SCF/ MPCD considers the compressibility contribution after particleto-field projection, which has the effect of smoothing. Instead, we may consider field profiles for all particle types, Fig. 10A, which are only defined at lattice positions of the field grid, and have been projected onto one direction, perpendicular to the membrane, to enable a direct comparison. These profiles clarify that solvent enrichment is completely absent in the coarser field representation. We thus conclude that inconsistencies between particle forces and density fields, as a result of finite-difference
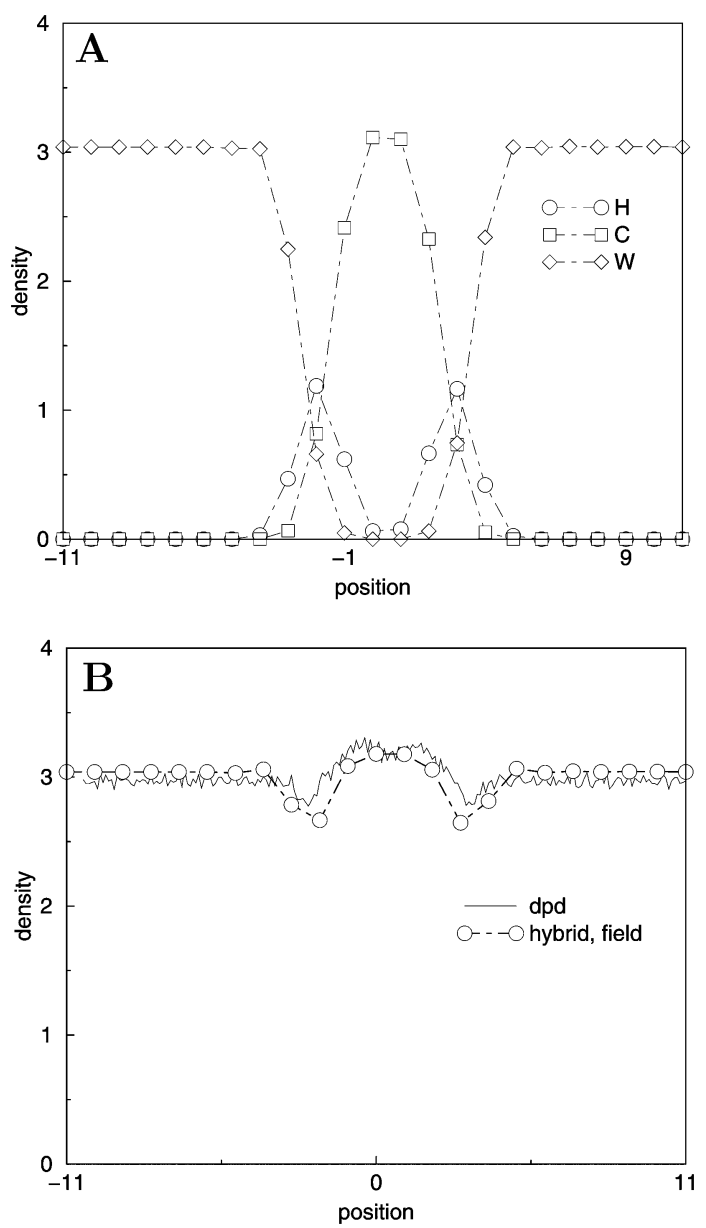

Fig. 10 (A) The density fields $\phi_{H}(\mathbf{r}), \phi_{C}(\mathbf{r})$ and $\phi_{W}(\mathbf{r})$, projected on a direction perpendicular to the membrane and averaged over the same number of snapshots as before, for the hybrid simulation $\left(l=1.0 r_{\mathrm{c}}\right)$. Symbols denote lattice positions, dashed lines are drawn to guide the eye. (B) The same for the total density field $\phi(\mathbf{r})=\phi_{H}(\mathbf{r})+\phi_{C}(\mathbf{r})+\phi_{W}(\mathbf{r})$. The total concentration profile for standard DPD derived from particle positions is added to enable direct comparison. For completeness, we note that a complete overlap of the MD-SCF/MPCD and DPD profiles is not to be expected, as the methods are based on different degree of coarsening. 
(FD) gradient calculation and subsequent interpolation, affect and slightly shift the force balance compared to a pure particle or field description, promoting increased occupancy away from the dividing interfaces and thus resulting in somewhat steeper interfacial profiles. This effect is most significantly for the thin $\mathrm{H}$-rich region close to the water-lipid interface. Focussing on the profile for the total field $\phi(\mathbf{r})$, see Fig. $10 \mathrm{~B}$, we find that the compressibility in both models, DPD and the new hybrid, is indeed comparable. We thus conclude that the differences in the particle profiles are mainly due to coarsening and the way forces are computed using the field grid.

Reducing the field discretisation parameter $l$ should improve the representability of rapidly varying density fields on the grid (note that the thickness of the membrane is $\sim 5 r_{\mathrm{c}}$ ) and increase the accuracy with which particle forces are obtained from projected density fields by FD and interpolation. We thus also simulated for the same thermodynamic parameter set using a reduced $l=0.75 r_{\text {c }}$ and $a=2 l$. The calculated average of 1.27 particles per lattice cell for this $l$ indicates that there will be an increased sensitivity to minute particle displacements in the projection algorithm, giving rise to quickly fluctuating fields, and thus to fluctuating forces, so we start close to the equilibrium structure. Starting from a pre-assembled membrane, we find that the mean-field free energy $W[\phi]$ indeed rapidly converges to a constant value. Comparing the averaged concentration profiles and the profiles for DPD, see Fig. 11, shows that increasing the field resolution indeed provides a better match between MD-SCF/MPCD and DPD, as expected when the coarsening is reduced. We thus conclude that the hybrid and DPD model for the lipids are consistent for this set of hybrid (thermodynamic) parameters.

Analysing the MD-SCF/MPCD dynamics. Next, we focus on the dynamics of structure coarsening. The 22-cube system simulated by the new hybrid MD-SCF/MPCD method for $\kappa_{\mathrm{H}}=5.0$, see the

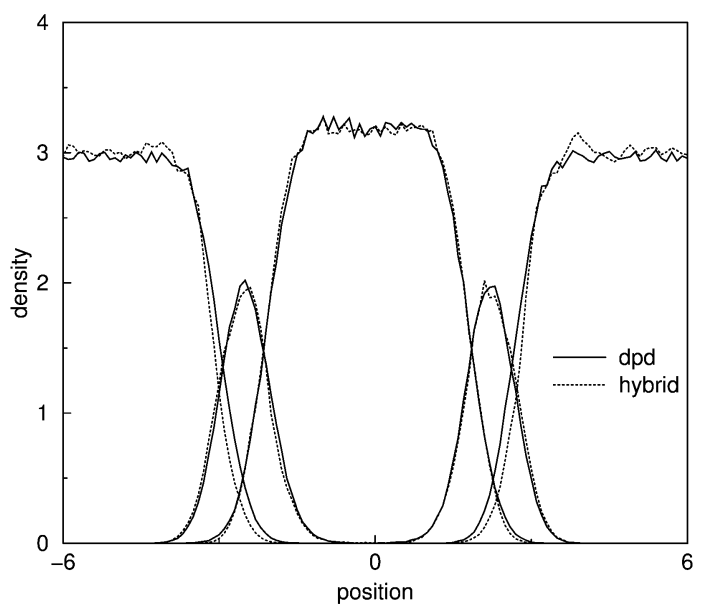

Fig. 11 Comparison of the concentration profiles of a self-assembled membrane simulated using standard DPD (solid lines) with a pre-assembled membrane for the new hybrid model, $l=0.75 r_{\mathrm{c}}$ and $a=2 l$ (dashed lines). The profiles for solvophilic head $(H)$, solvophobic tail $(C)$ and solvent $(W)$ particles were determined from binning instantaneous particle positions and averaging over a large number of simulation snapshots.
A

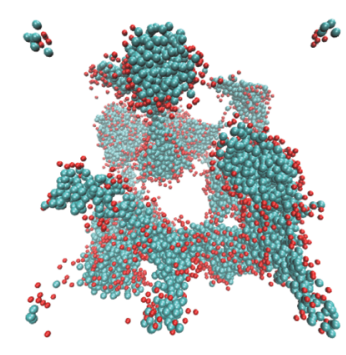

$\mathrm{C}$
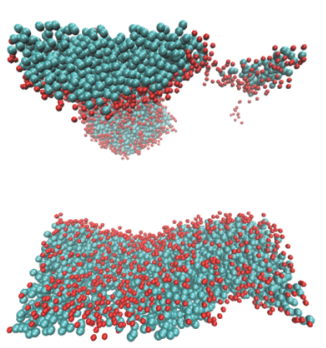

B

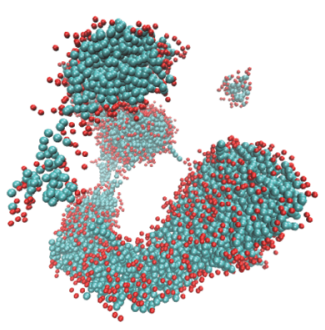

D
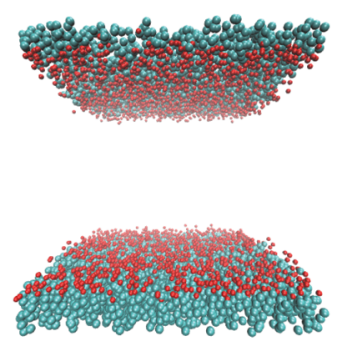

Fig. 12 Snapshots after $10^{3}(\mathrm{~A}), 2 \times 10^{3}(\mathrm{~B}), 5 \times 10^{3}(\mathrm{C})$ and $10^{4}(\mathrm{D})$ time steps of simulation for the new hybrid MD-SCF/MPCD method $\left(\Delta t^{*}=0.01\right)$, showing the structural evolution of the system towards an aligned membrane, which is the same as in Fig. 7 (bottom, right). For clarity, only $\mathrm{H}_{3}\left(\mathrm{C}_{4}\right)_{2}$ lipids are shown, with red/blue representing $\mathrm{H} / \mathrm{C}$ particles.

final structure in Fig. 7C, and the corresponding structure evolution in Fig. 12, serves as a starting point or reference. For brevity, we denote a planar membrane along two Cartesian basis vectors as an 'aligned' membrane, and the MD-SCF method of Milano et al. as the original hybrid method, whereas the new MD-SCF/MPCD method is the new hybrid method. Fig. 13 shows simulation snapshots for the same parameter set, but now simulated using the original hybrid method, which employs an Andersen thermostat for maintaining a constant temperature. ${ }^{26}$ In particular, Velocity Verlet (12) with the same time step $\left(\Delta t^{*}=0.01\right)$ is combined with an Andersen thermostat, with a collision frequency of $5 \mathrm{ps}^{-1}$, and the same homogeneous lipid-water mixture is used as a starting structure. Whereas the formation of a planar membrane in the reference (new hybrid) model is complete after $10^{4}$ time steps, see Fig. 12, the structure simulated using the original hybrid method is a collection of lipid micelles at this stage, see Fig. 13. With time, these micelles merge with others to form elongated cylinders that may or may not be interconnected, see snapshot after $10^{5}$ time steps, while a single and stable planar membrane, under an angle with the Cartesian basis, containing a large circular protrusion has formed after 3-4 times longer simulation $\left(4 \times 10^{5}\right.$ time steps).

The comparison of the evolution of the MFs, see log-normal plot in Fig. 14, confirms that structure coarsening takes place on a different pace between the two hybrid models. This difference is particularly apparent in the early stages, where convective flows are known to speed up structure coarsening in reality, even in the absence of external flow fields. The initial high values for the curvature and surface area, see Fig. 14, can be attributed to the formation of many isolated micelles from a 


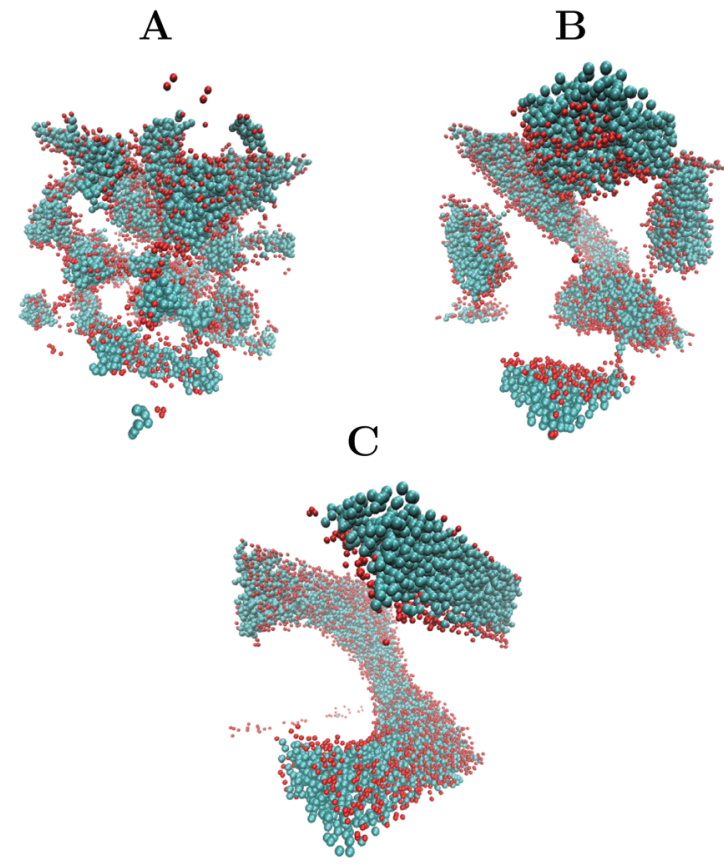

Fig. 13 Snapshots after $10^{4}(\mathrm{~A}), 10^{5}(\mathrm{~B})$ and $10^{6}(\mathrm{C})$ time steps of simulation for the original SCF/MD scheme $\left(\Delta t^{\star}=0.01\right) .{ }^{29}$ Both the simulation volume and the thermodynamic parameters are the same as in Fig. 7. The equilibrium structure is a slightly bended and considerably tilted membrane, that contains a stable and large circular protrusion. For clarity, only $\mathrm{H}_{3}\left(\mathrm{C}_{4}\right)_{2}$ lipids are shown, with red/blue representing $\mathrm{H} / \mathrm{C}$ particles.

homogenous mixture, whereas the relatively fast reduction for the new hybrid method is a signature for micelle coalescence, a process that is accelerated by hydrodynamic interactions between them. The different slopes for both hybrid methods indicate that hydrodynamics also plays a role at later stages. For the original method, the MF related to integral mean curvature decays slowly with time, signalling the formation of a flat membrane from (cylindrical) micelles, albeit that the final value is positive due to the presence of the protrusion. The final MF related to the total surface area for this structure is clearly higher than for the aligned membrane.

To evaluate which factor is most significant in the phase separation kinetics, i.e. accounting for effective collisions in MD-SCF/MPCD or the global Andersen thermostat in MD-SCF, we also performed MD-SCF simulations with an Andersen thermostat for a reduced collision frequency of $2 \mathrm{ps}^{-1}$, as well as MD-SCF without any thermostat. In the latter case, we simulate a microcanonical (NVE), for which hydrodynamic interactions should be correctly represented, instead of the usual canonical $(N V T)$ ensemble. Since part of the potential energy, which is high for the starting mixed system with many lipid-water contacts, flows to kinetic energy during simulation, however, trajectories may become unstable for a constant time step $\Delta t^{*}$. Such instability originates from intra-molecular interactions, as the intermolecular interactions are soft.

The evolution of the non-ideal free energy $W[\phi]$ for MD-SCF without a thermostat and two small (constant) values of the time step, $\Delta t^{*}=5 \times 10^{-4}$ and $\Delta t^{*}=10^{-4}$, are shown in Fig. 15,
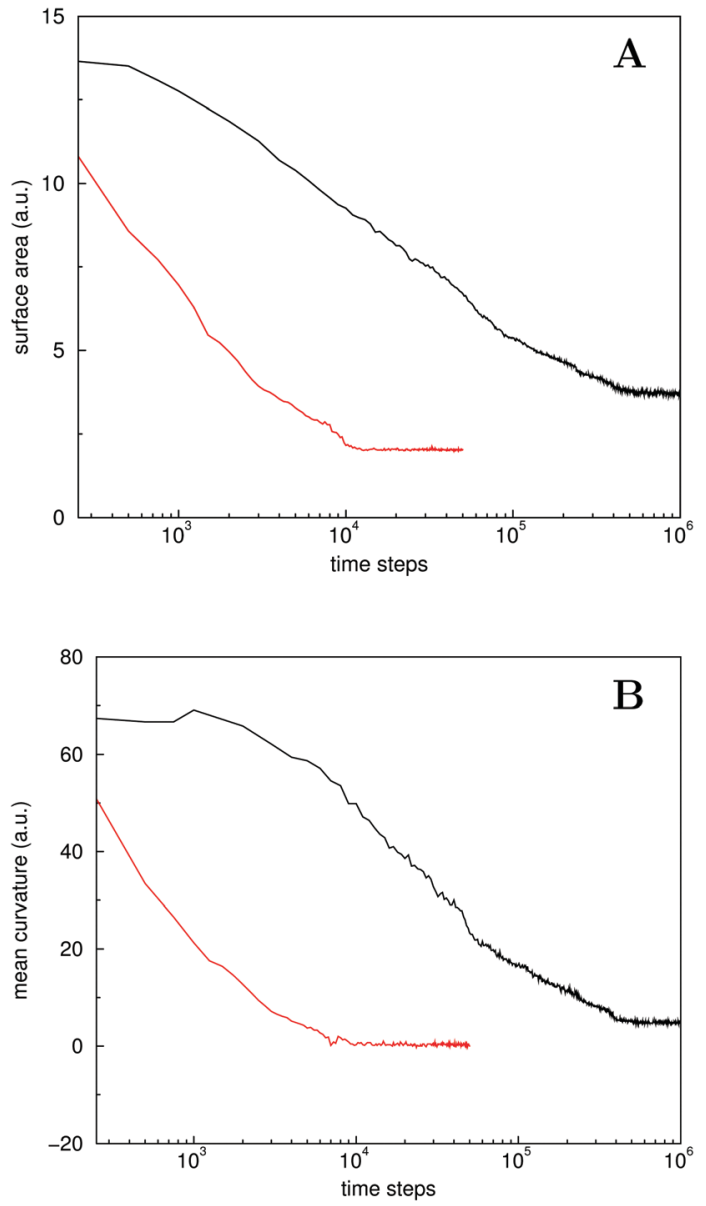

Fig. 14 Evolution of the MFs related to the (total) surface area (A) and integral mean curvature (B), for the original MD-SCF approach of Milano et al. ${ }^{29}$ (black) and for the new model based on MPCD (red). Note that the time steps $\Delta t^{*}$ are equal in both methods and that the horizontal time axis is plotted on a logarithmic scale. The caption of Fig. 8 provides more information about the MFs calculation.

together with data for MD-SCF with an Andersen thermostat (update frequency $5 \mathrm{ps}^{-1}$ ) and MD-SCF/MPCD, both obtained for $\Delta t^{*}=0.01$. To enable a direct comparison, the energy $W[\phi]$ (logarithmic scale) has been plotted versus time $t^{*}$, with $t^{*}=\Delta t^{*} \cdot n$ and $n$ the number of time steps in the simulation. As expected, the temperature (kinetic energy) in the nonthermostatted simulations rises with progressing phase separation and eventually these simulations become unstable, as can be observed from the rising $W[\phi]$. Further decreasing the time step to $\Delta t^{*}=5 \times 10^{-5}$, or starting from a system that is thermostatted for a short while, does not resolve this issue. Nevertheless, Fig. 15 clearly shows an enhanced reduction of $W[\phi]$ during the initial stages in the absence of a thermostat, illustrating that it is indeed the use of the Andersen thermostat that slows down the kinetics.

Turning to the effect of the collision frequency in MD-SCF simulations with an Andersen thermostat, we compare the evolution of the non-ideal free energy $W[\phi]$ for the two considered update frequencies, see Fig. 16A. We observe that the kinetics is enhanced for the lowest collision frequency. Since a reduced 


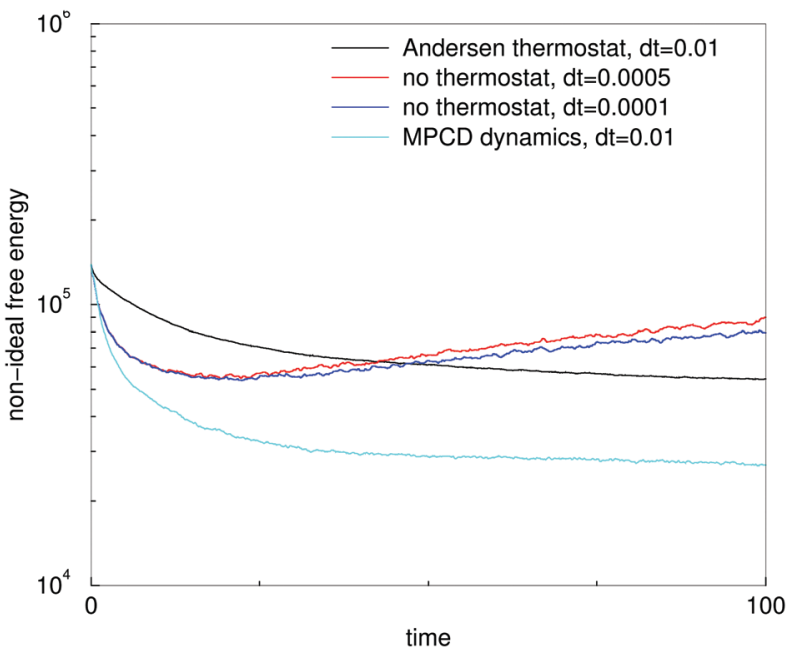

Fig. 15 Comparison of the evolution of the non-ideal free energy $W[\phi]$ simulated using the Andersen thermostat (black) and without thermostat, for two values of the time step $\Delta t^{*}: 0.0005$ (red) and 0.0001 (blue). As a reference, the data for the MD-SCF/MPCD simulation is added (cyan). The energy (logarithmic scale) is plotted versus time $t^{*}$, with $t^{*}=\Delta t^{*} \cdot n$ and $n$ the number of time steps. All systems were simulated for $10^{6}$ time steps in total from the same starting structure, a lipid/water mixture.

collision frequency implies that the velocities of fewer particles are rescaled every time step, this finding agrees with the results of MD-SCF without a thermostat. Concentrating on the long-time behaviour, $W[\phi]$ is seen to converge to very comparable values, showing that the simulated structures are stable and suggesting that the stable structure does not depend on the particular trajectory. However, the stable structure for lowest collision frequency is the interconnected aligned membrane-patches shown in Fig. 16B, rather than the tilted planar membrane with a protrusion obtained for the original collision frequency, see Fig. 13. The finding of two different structures with very comparable $W[\phi]$ suggests that both are metastable states of the system.

Self-assembly of a tilted planar membrane can be regarded as a sign of frustration and a hint that a tensionless membrane is incommensurate with the size of the simulation volume. To further investigate whether this plays a role, we have also considered the stability and non-ideal free energy of the proposed equilibrium structure, an 'aligned' membrane, which was pre-assembled by positioning lipids in a membrane-like arrangement perpendicular to the $z$-direction as a whole, and equilibrated using MD-SCF with the original Andersen thermostat. Direct comparison of $W[\phi]$ for the self-assembled membrane and this pre-assembled membrane, see Fig. 17, shows that $W[\phi]$ for the pre-assembled aligned membrane is the lowest, although the difference is not very large. This result suggests that the self-assembled membranes discussed in the previous paragraphs are formed as a result of kinetic trapping: micellar proto-structure connects by proximity, as a result of diffusion, into an initial space-spanning structure. Consequently, minimising the line tension, by reducing the overall curvature of the structure and fusing all holes into one circular protrusion (for the original collision frequency) or a connection region (for the reduced collision frequency) to form a flat but incomplete
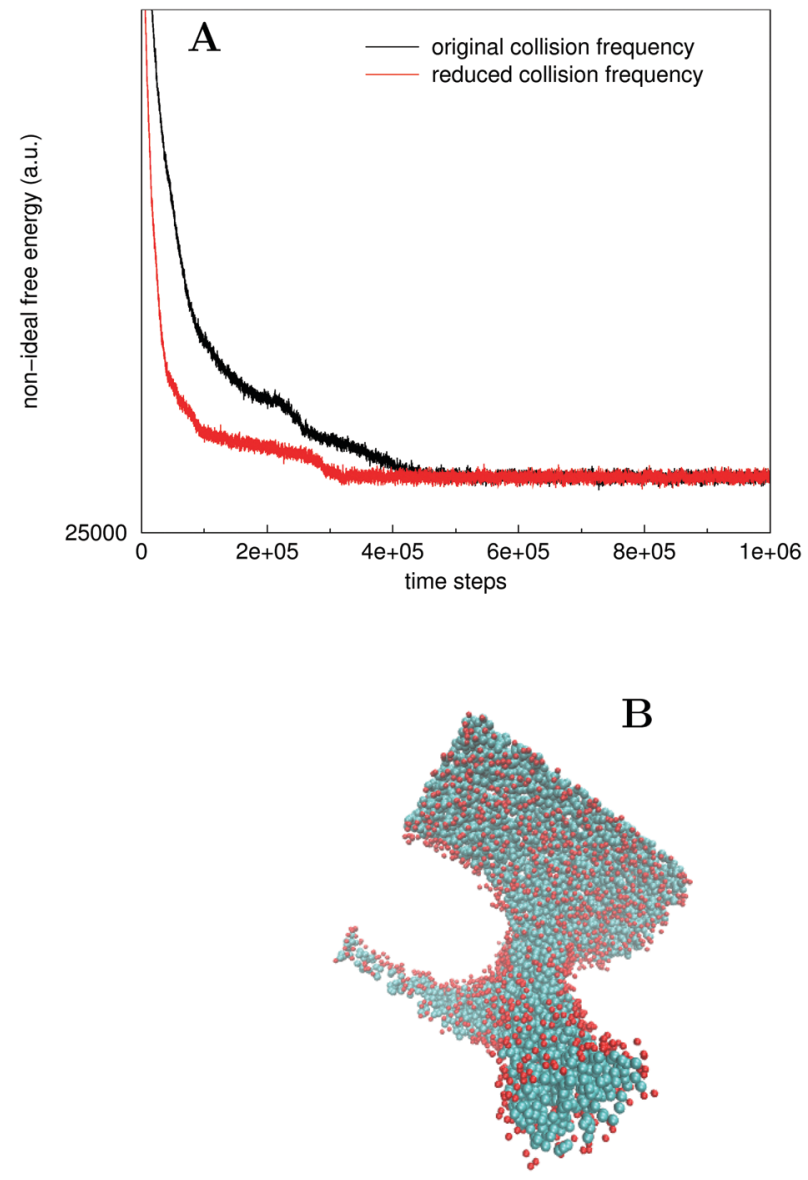

Fig. 16 (A) Comparison of the evolution of the non-ideal free energy $W[\phi]$ simulated using the original collision frequency $5 \mathrm{ps}^{-1}$ (black) and a reduced frequency $2 \mathrm{ps}^{-1}(\mathrm{red})$ in the Andersen thermostat $\left(\Delta t^{\star}=0.01\right)$. (B) Stable structure obtained after $10^{6}$ time steps for the simulation with a reduced collision frequency. For clarity, only $\mathrm{H}_{3}\left(\mathrm{C}_{4}\right)_{2}$ lipids are shown, with red/blue representing $\mathrm{H} / \mathrm{C}$ particles.

membrane, is apparently more likely than the formation of a complete membrane that is fully aligned with two Cartesian directions. Apart from the weak thermodynamic driving forces for such restructuring, one should again be aware that the condition of 3D periodicity affect energetic barriers and thus the transitions between accessible states. Overall, we conclude that these incomplete membranes are metastable states of the system, and do not form as a result of a parameter mismatch.

Focussing on the concentration profiles for MD-SCF with an Andersen thermostat, determined from the equilibrated preassembled membrane, we find that they are in good agreement with the profiles for the membrane simulated using DPD, again apart from excess solvent close to the membrane-solvent interface, see Fig. 18. Considering the total particle concentration, like before, we find that the profile (not shown) in the vicinity of the lipid-water interface has a very similar signature as in Fig. 9B for MD-SCF/MPCD, albeit that the amplitude of the oscillations is somewhat smaller.

Finally, we analyse the diffusion rates for all systems in this section, i.e. for systems simulated using DPD, MD-SCF with an 


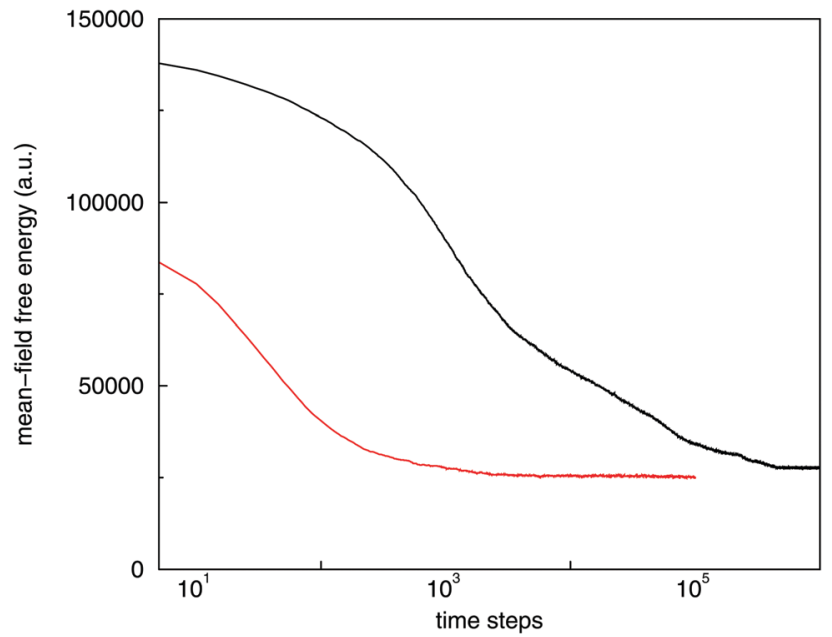

Fig. 17 Comparison of the evolution (logarithmic time axis) of the meanfield free energy $W[\phi]$ for the self-assembled membrane shown in Fig. 12 (black) and a planar pre-assembled (or aligned) membrane (red).

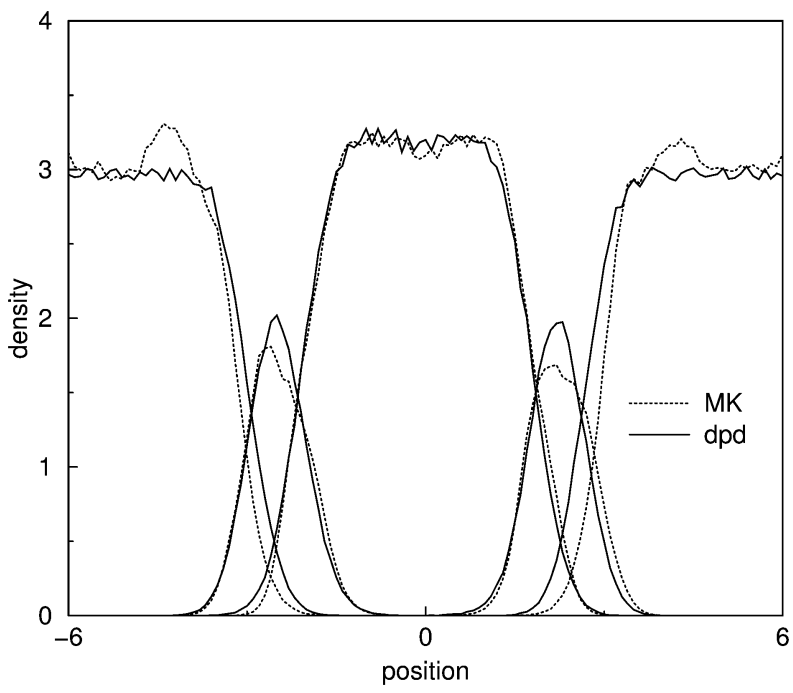

Fig. 18 Comparison of the concentration profiles of a self-assembled membrane simulated using standard DPD (solid lines) and a preassembled membrane (dashed lines) for the model of Milano et al. ${ }^{29}$ The profiles for solvophilic head $(\mathrm{H})$, solvophobic tail $(\mathrm{C})$ and solvent (W) particles were determined from binning instantaneous particle positions and averaging over a large number of simulation snapshots.

Andersen thermostat, or MD-SCF/MPCD. Values for the diffusion constant $D^{*}$ are obtained from a linear fit of the mean-square displacement (MSD) for long times, with $D_{\mathrm{w}}{ }^{*}$ the diffusion coefficient for water particles and $D_{1}^{*}$ the diffusion coefficient for the centre of mass of the lipid chains, see Table 3. As these values are calculated from limiting behaviour, lipid diffusion in the solvent phase is not considered in this analysis. We do observe fast, anomalous diffusion in the initial stages, where lipids start to self-organise, but at later stages the MSD adopts an (approximately) linear relation with simulation time. The findings confirm that the kinetics in MD-SCF/MPCD is considerably enhanced compared to the MD-SCF of Milano et al.
Table 3 Diffusion rates for the solvent $D_{\mathrm{w}}{ }^{*}$ and the centre of mass of the lipids $D_{1}^{*}$. These values are extracted from a linear fit of the mean-square displacements that were measured during the last half of $2 \times 10^{5}$ (DPD and MD-SCF/MPCD) or $10^{6}$ (MD-SCF) trajectories

\begin{tabular}{lll}
\hline Method & $D_{\mathrm{w}}{ }^{*}$ & $D_{1^{*}}$ \\
\hline DPD & 0.14 & 0.0066 \\
MD-SCF, Andersen & 0.035 & 0.0018 \\
MD-SCF/MPCD, $f_{\text {up }}=5$ & 0.14 & 0.096
\end{tabular}

Moreover, the different diffusion rates for lipids in DPD and MD-SCF/MPCD signal that particularly the lipid kinetics is unmatched between the two models, which agrees well with earlier observations that the Schmidt number in DPD is always close to one. ${ }^{30}$

Lipid-solvent system in increased volumes. Before we concentrate on the results of hybrid simulation for larger simulation volumes, we shortly discuss the property of the considered DPD lipid representation to promote the positioning of head particles next to other head particles rather than to water particles (since $a_{\mathrm{HH}}<a_{\mathrm{HW}}$ ), which stimulates the formation of multilayer structures. ${ }^{55}$ Indeed, performing a DPD simulation with a lipid content that is commensurate with a tensionless membrane along the $x y$ directions in a rectangular simulation volume $\left(L_{x}=L_{y}, L_{z}<L_{x} / 2\right)$, starting from randomly positioned lipids, the (quasi)equilibrium structure is the defected double bilayer shown in Fig. 19. This finding is consistent with $a_{\mathrm{HH}}<a_{\mathrm{HW}}$ and an approximate matching of double membrane structure along the shortest dimension of the simulation volume. We may expect a competition between single- and multilayer structure, with one layer reflecting a lipid bilayer, also in the hybrid model.

Next, we consider the hybrid model for a larger cubic volume $V^{*}\left(L_{x, y, z^{*}}=32\right)$, containing $n_{1}=1617$ lipids and $n_{\mathrm{w}}=80517$ solvent particles $(N=98304)$. The lipid content is deliberately chosen consistent with a flat tensionless (DPD) membrane that spans the cubic simulation volume along two Cartesian directions. Before we consider the results, we note that the periodic

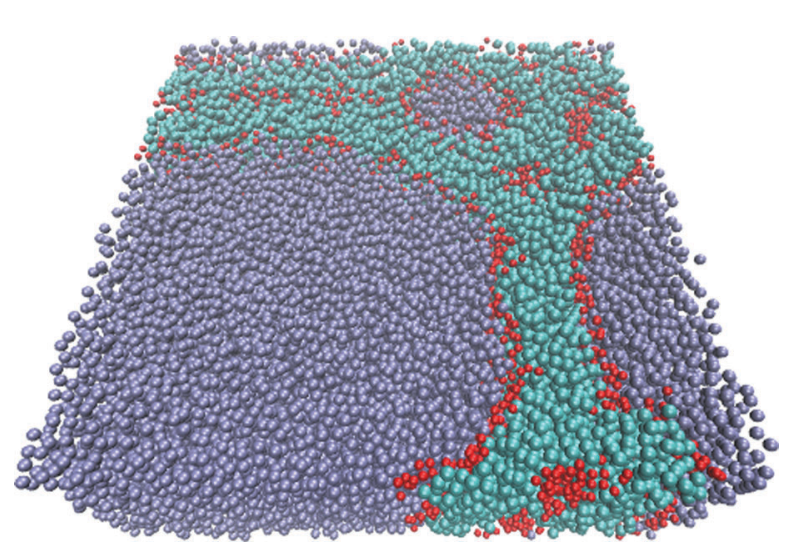

Fig. 19 Snapshot after $2 \times 10^{5}$ time steps of DPD simulation $\left(\Delta t^{\star}=0.02\right)$ in a $30 \times 30 \times 12$ simulation volume (all in units of $r_{\mathrm{c}}$ ). The simulation volume contains $n_{1}=1422 \mathrm{H}_{3}\left(\mathrm{C}_{4}\right)_{2}$ lipids and $n_{\mathrm{w}}=16758$ solvent (W) particles $(N=32400)$. All particles are shown, with red/blue/iceblue representing $\mathrm{H} / \mathrm{C} / \mathrm{W}$ particles. 
boundary conditions play an important role in the forthcoming discussion. In particular, any free-floating newly formed bicelle (i.e. an isolated round membrane patch) will experience an energetic penalty due to its edge domain or rim. As a result of the competition between this and other factors, bicelles that grow beyond a critical size become unstable and favour a transition into a stable vesicle, albeit that both states remain separated by an energetic barrier. Using periodic boundary conditions, however, this energetic penalty associated with the growing rim can alternatively be avoided when a large bicelle connects with its mirror image, over the simulation volume boundaries, to become one space-spanning (flat) membrane. This process will not be prevented by a sudden increase of the surface tension, as the selected lipid concentrations agrees well with a tensionless membrane; moreover, the disappearance of the rim enhances the stability of such a membrane when compared to a bicelle of the same size. Thus, especially for simulation volumes that are larger than this critical bicelle size, one may expect a pathway-dependent formation of space-spanning membranes or vesicular structures.

Depending on the starting conditions, we indeed find two structures with very similar non-ideal free energy $W[\phi]$ : a doublelayer structure that spans the simulation volume in one of the Cartesian directions (and topologically analogous of a vesicle that is flattened to span the simulation volume along one direction, see Fig. 20A) forms when starting from lipids that are randomly distributed in the whole simulation volume, while a single-layer aligned (planar) membrane forms upon distributing the lipids initially only in half of the volume (see Fig. 20B). Assuming that the first is a planar membrane of thickness $2 d$, capped by two half cylinders with radius $d$, we can use volume conservation to determine the length $\tilde{L}$ of its planar part, as

$$
\tilde{L}=\frac{1}{2}(L-\pi d)
$$

with $L$ the length of the (cubic) simulation volume. Using this relation, we can write the Helfrich Hamiltonian for the two cases as

$$
\begin{gathered}
\mathcal{H}_{1}=2 \sigma_{1} L^{2} \\
\mathcal{H}_{2}=\sigma_{2}\left(L^{2}+\pi d L\right)+\frac{\pi L \kappa_{\mathrm{c}}}{d} .
\end{gathered}
$$

Since both structures have the same non-ideal free energy, we assume that also $\mathcal{H}_{1}=\mathcal{H}_{2}$ for this $L$. Filling in $L=32 r_{\mathrm{c}}$, as well as $d=5.5 r_{\mathrm{c}}$ and $\kappa_{\mathrm{c}}=25 k_{\mathrm{B}} T$, the earlier determined values, we find that $\sigma_{1}^{*}=0.77 \sigma_{2}^{*}+0.22$. The number of lipids was selected to provide a tensionless aligned membrane, i.e. small $\sigma_{1}^{*}<1.0$, so we find that $\sigma_{2}^{*} \leq \sigma_{1}^{*}$ as expected for a structure that selfassembles from a completely dispersed lipid/water mixture. Moreover, since (24) additionally indicates that $\mathcal{H}_{1}<\mathcal{H}_{2}$ for $L<32 r_{\mathrm{c}}$ and $\mathcal{H}_{2}<\mathcal{H}_{1}$ for $L>32 r_{\mathrm{c}}$, this analysis agrees with our previous reasoning and explains the computational finding that an aligned planar membrane usually forms by self-assembly in smaller simulation volumes, whereas a multi-layer structure is consistently found in larger simulation volumes. We note, however, that the quantitative value of this analysis is restricted,

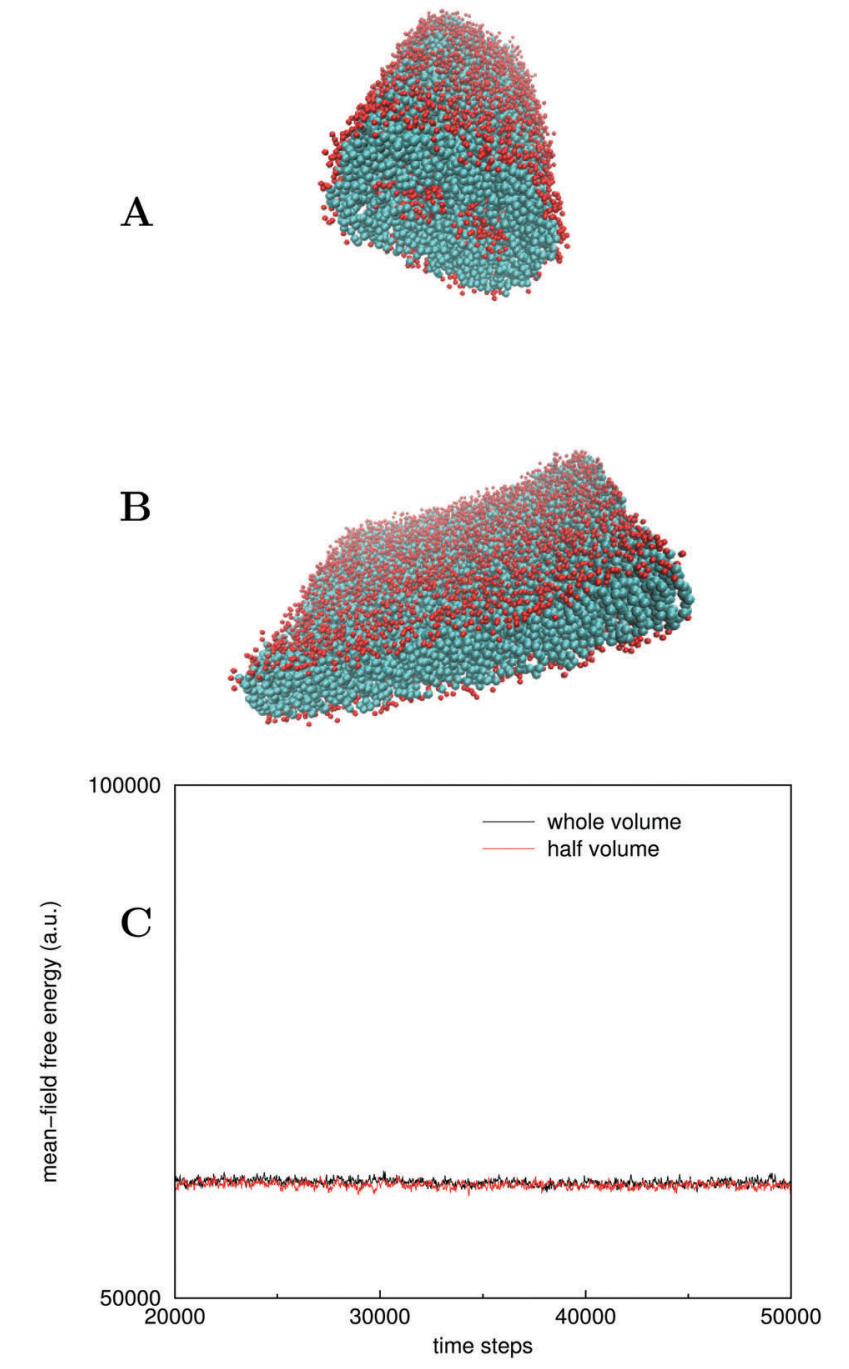

Fig. 20 Snapshots after $5 \times 10^{4}$ time steps of hybrid simulation in a $32 \times 32 \times 32$ simulation volume (all in units of $r_{c}, n_{l}=1617, \Delta t^{*}=0.01$ ). In both cases, the simulations are started from a mixture of lipids and water, with lipids initially randomly dispersed throughout: the whole simulation volume (A) or half of the simulation volume (B). For clarity, only $\mathrm{H}_{3}\left(\mathrm{C}_{4}\right)_{2}$ lipids are shown, with red/blue representing $\mathrm{H} / \mathrm{C}$ particles. (C) comparison of the evolution (logarithmic time axis) of the mean-field free energy $W[\phi]$ for the self-assembled structures shown above.

as we should more carefully single out boundary effects before drawing any general conclusions.

\section{Improved force calculation}

The compact (6-point) discrete gradient operator, see Appendix A for details, used to calculate particle forces from the mean-field potentials, has the advantage of being computationally efficient, but compactness may also bring along undesired effects. This can be seen clearly from a MD-SCF/MPCD simulation that starts from a pre-assembled spherical liposome containing a large hole/protrusion, see Fig. 21A. With time, the hole nicely disappears, a process that is driven by the undesired line tension associated with the rim of the hole, but meanwhile, at a larger scale, the vesicle also adapts its shape, and transforms into an 


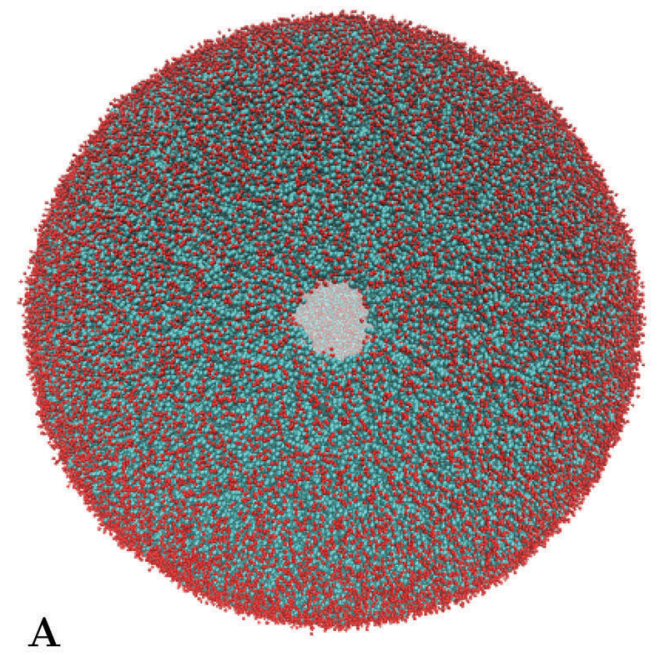

A

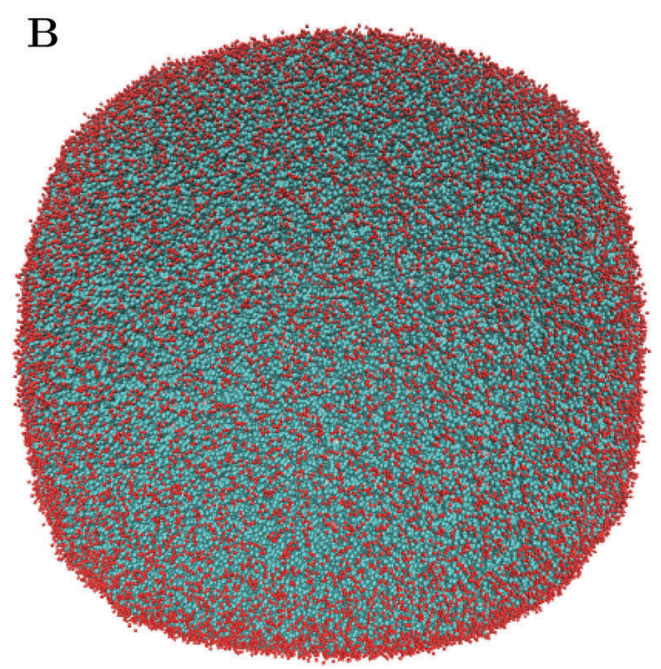

Fig. 21 Snapshots after 200 (A) and 5200 (B) time steps of MD-SCF/ MPCD simulation in a $100 \times 100 \times 100$ simulation volume (all in units of $r_{\mathrm{C}}$, $n_{1}=36236, N=3 \times 10^{6}, \Delta t^{\star}=0.01$ ). The discrete gradient operator of Appendix $\mathrm{A}$ is used in the force calculation. Only lipid particles are shown for clarity, with red/blue representing $\mathrm{H} / \mathrm{C}$ particles.

unexpected, cubic vesicle. Although facets may have a physical cause, we consider this finding a sign that the discrete gradient operator is slightly anisotropic, the effect of which is most clearly seen in larger lipid structures. Here, we redo some of the simulations using a more isotropic discrete gradient operator taken from literature. For simplicity, we focus on long-term behaviour rather than formation pathways. All thermodynamic and numerical parameters are the same as in the referred simulations.

Fig. 22 shows the equilibrated results in a small cubic volume $V^{*}\left(L_{x, y, z^{*}}=22\right)$ for both MD-SCF/MPCD and MD-SCF based on an Andersen thermostat, compare to the result shown in Fig. 7C and the final snapshot (C panel) in Fig. 13. For both methods, an aligned planar membrane forms eventually. Apparently, the choice of the gradient operator is not decisive for the new hybrid MPCD scheme, because of the small
A
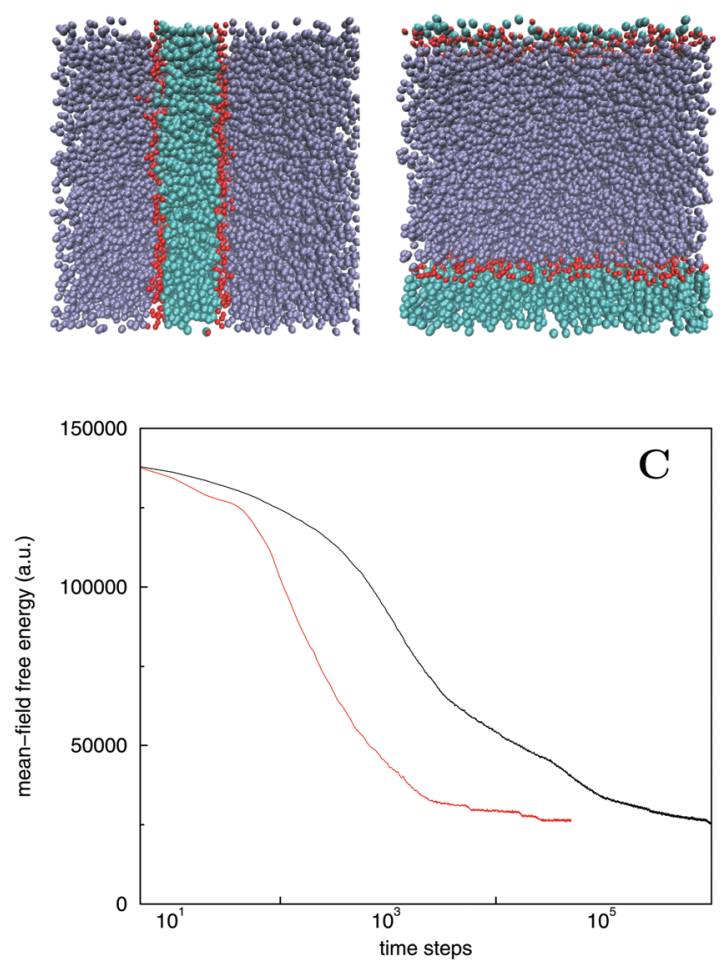

Fig. 22 Snapshots after $5 \times 10^{4}$ time steps of hybrid MPCD simulation (A) and after $1 \times 10^{6}$ time steps of MD-SCF with an Andersen thermostat (B). In both cases, flat membranes spanning the $22 \times 22 \times 22$ simulation volume (all in units of $r_{\mathrm{c}}, n_{\mathrm{l}}=764, \Delta t^{*}=0.01$ ) are formed, starting from a mixture of lipids and water, with lipids initially randomly dispersed. All particles are shown, with red/blue/iceblue representing H/C/W particles. (C) Comparison of the evolution (logarithmic time axis) of the mean-field free energy $W[\phi]$ for the self-assembled membranes shown above (red: MPCD, black: Andersen thermostat).

simulation volumes, although a planar membrane takes somewhat longer to form. For the original hybrid scheme, ${ }^{29}$ however, we find that the isotropy of the discrete gradient operator does matter. Instead of a tilted perforated membrane that we found before, we obtain an aligned, complete membrane. Clearly, another factor plays a role in the formation of a tilted structure, which is the directional bias to the forces introduced by a less isotropic gradient. Apparently, this bias promotes the formation of a metastable state in the form of a tilted bilayer, see Fig. 7 (bottom, right).

The non-ideal free energy for the MD-SCF/MPCD and the MD-SCF with an Andersen thermostat should be the same for this aligned membrane for us to firmly conclude that MPCD indeed samples a correct ensemble. Close comparison shows that the $W[\phi]$, which is noisy as a result of thermal fluctuations, for the two different simulations are close but slightly offset. To understand the origin of this offset, we merged both methods by continuing the MD-SCF/MPCD simulation of Fig. 22 by MD-SCF with the usual Anderson thermostat. We find, see Fig. 23, that the simulation temperature in the first part, simulated using MD-SCF/MPCD, is slightly higher than $T^{*}=1$. 

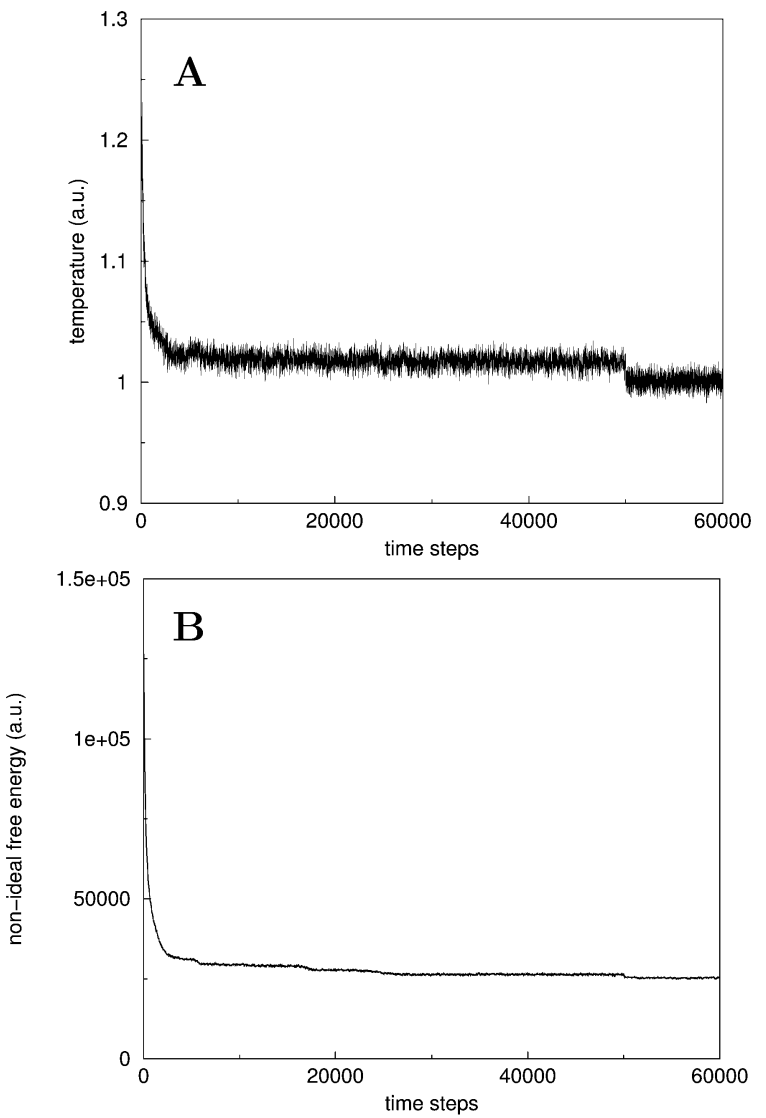

Fig. 23 Evolution of the temperature $T^{\star}(\mathrm{A})$ and non-ideal free energy $W[\phi]$ (B) for a special case. In this simulation, the first $5 \times 10^{4}$ time steps are simulated using MD-SCF/MPCD, see Fig. 22 for details. These results are passed on to MD-SCF, with an Andersen thermostat and a collision frequency of $5 \mathrm{ps}^{-1}$, which is used to simulate the system for the last $10^{4}$ time steps.

Switching after $5 \times 10^{4}$ time steps, see Fig. 23, gives rise to an instantaneous drop in the temperature and in the non-ideal free energy. In particular, the small offset in $W[\phi]$ vanishes instantaneously and the value is the same in both simulations. Although one key difference is in the thermostatting - in MD-SCF/MPCD, thermostatting is only applied at collision time steps, while the Andersen thermostat in MD-SCF is active at every time step - we conclude from our stability analysis (see Method section) that the origin of this decrease lies in the reduced particle mobility for the same projection frequency $f_{\text {up }}$. Indeed, if we use the same procedure as before to calculate diffusion coefficients for the new gradient, we find $D_{\mathrm{w}}{ }^{*}=0.033$ and $D_{\mathrm{l}^{*}}=0.0023$ for MD-SCF and $D_{\mathrm{w}}^{*}=0.14$ and $D_{\mathrm{l}}^{*}=0.13$ for MD-SCF.

From these results, we conclude that the introduction of a more isotropic gradient resolves the previously obtained mismatch in terms of stable structures obtained by both hybrid methods. The time scales at which these stable structures form still significantly depend on the selected model, i.e. MPCD or an Andersen thermostat, for the system dynamics. Redoing the simulation for the punctured vesicle, see Fig. 21, using this isotropic discrete gradient operator, provides a closed and perfectly spherical stable vesicle, see Fig. 24 .

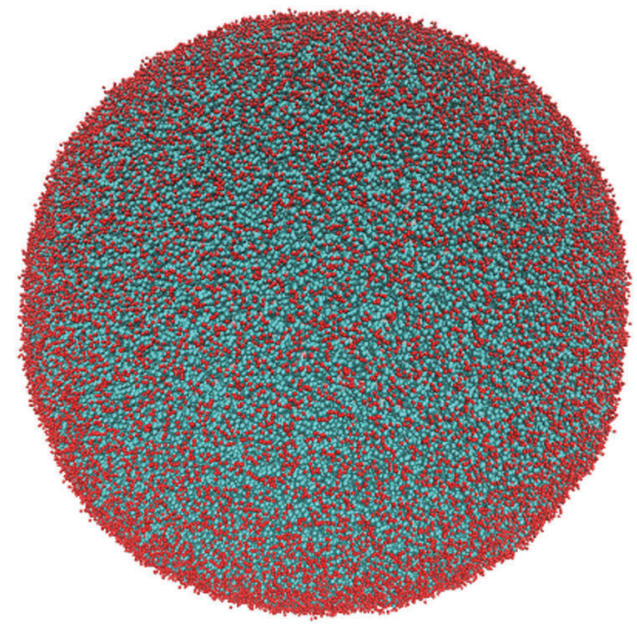

Fig. 24 Snapshot after 4000 time steps of MD-SCF/MPCD simulation in a $100 \times 100 \times 100$ simulation volume (all in units of $r_{\mathrm{C}}, n_{\mathrm{l}}=36236, N=3 \times 10^{6}$, $\Delta t^{\star}=0.01$ ), showing a closed up protrusion in a perfectly spherical vesicle. The simulation was started from the same initial structure as in Fig. 21, and the new discrete gradient operator of Appendix $B$ is used in the force calculation. The vesicle was determined stable based on the non-ideal free energy reaching a plateau value. Only lipid particles are shown for clarity, with red/blue representing $\mathrm{H} / \mathrm{C}$ particles.

Although we did not observe a significant effect of anisotropy in the MD-SCF/MPCD results for larger volumes, we also analyse the effect of replacing the discrete gradient operator for some of the other previously simulated systems. In a larger cubic volume $V^{*}\left(L_{x, y, z^{*}}=32\right)$, we again observe a competition between aligned (planar) membranes and alternative structures with equivalent mean-field free energy, see Fig. 25, indicative of the prominent role of the (periodic) boundary conditions for this case. Distributing the lipids initially only in half of the simulation volume gives rise to the formation of an aligned membrane, while starting a simulation from lipids distributed randomly over the whole volume gives rise to alternative structure. For the latter case, two type of structures are obtained after $10^{5}$ time steps, depending on the initial lipid distribution, one of which (structure 2) is very similar to the double-layer structure found for the old discrete gradient operator, see Fig. 20, however, in coexistence with a small lipid micelle. Apparently, the simulation pathway is too short to observe fusion of these two domains into a single structure. The alternative (structure 1), slightly higher in non-ideal free energy than structure 2 and coexisting with a larger lipid micelle, is a multi-layer vesicle. It can be anticipated that, in both cases, fusion into a single structure would produce the double-layer structure of Fig. 19, having a mean-field free energy that is equivalent to that of an aligned membrane. Overall, these findings show that the new discrete gradient operator slows down the formation kinetics compared to the old one. An intuitive understanding for this phenomenon is that the old discrete gradient operator gives rise to slightly enhanced diffusion along particular directions.

Finally, we challenge the isotropy of the new discrete gradient operator by starting from a pre-assembled curved bicelle or half vesicle, see Fig. 26. Simulating this setup using the original 
A
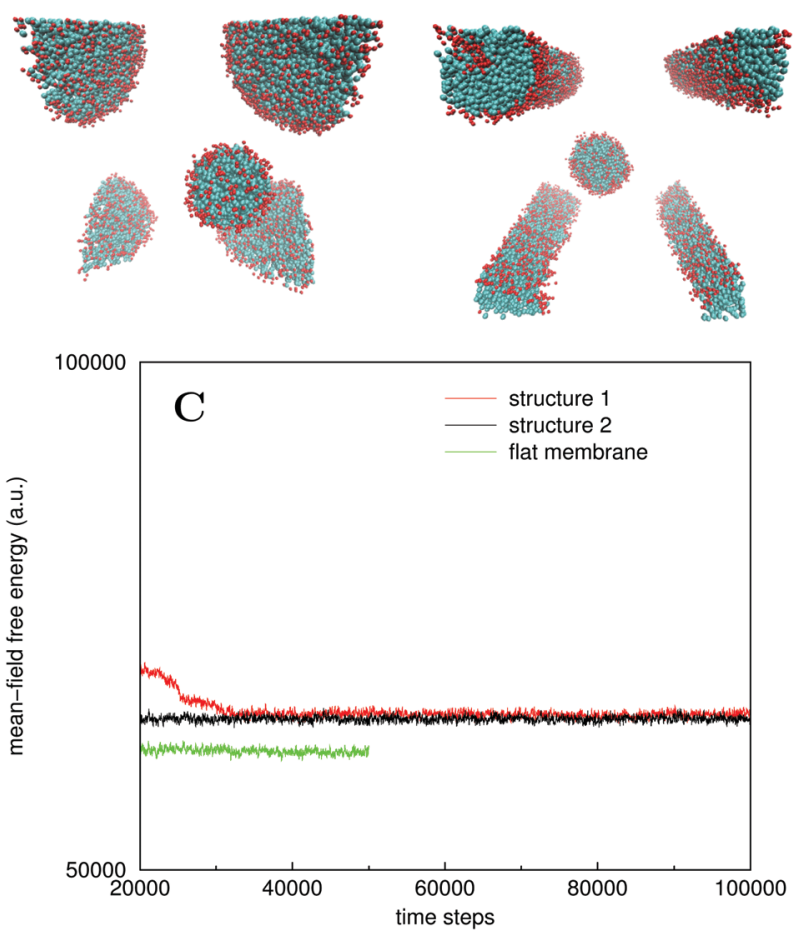

Fig. 25 Snapshots after $1 \times 10^{5}$ time steps of hybrid simulation in a $32 \times 32 \times 32$ simulation volume (all in units of $r_{\mathrm{c}}, n_{\mathrm{l}}=1617, \Delta t^{\star}=0.01$ ) for two different starting configurations. Both simulations are started from a mixture of lipids and water, with lipids initially randomly dispersed throughout the volume (A: structure 1; B: structure 2). For clarity, only $\mathrm{H}_{3}\left(\mathrm{C}_{4}\right)_{2}$ lipids are shown, with red/blue representing $\mathrm{H} / \mathrm{C}$ particles. (C) Comparison of the evolution (logarithmic time axis) of the meanfield free energy $W[\phi]$ for the self-assembled structures shown above. As a comparison, we have added a graph for the case where lipids are initially randomly dispersed throughout only half of the simulation volume, which self-assembles into the flat membrane shown in Fig. 19.

slightly anisotropic discrete gradient operator, we found that lipids along the initially circular rim experience anomalous diffusion as a result of angle-dependent forces, giving rise to a quick formation of a stable square rim (no closing up within the simulated pathway, results not shown). Concentrating on the more isotropic discrete gradient operator, we find that the closing up of the pore, resulting in a vesicular structure, is radially symmetric, and that a spherical vesicle is formed rather quickly, i.e. within $2 \times 10^{4}$ time steps. The radius of this vesicle, measured from the centre of mass to the outer leaflet, is roughly 18 (in units of $r_{\mathrm{c}}$ ), with 1564/2404 lipids in the inner/ outer leaflet. A recent coarse-grained molecular dynamics study for DMPC lipids corroborates that vesicle formation from such an initial structure should be spontaneous. ${ }^{56}$

Although the code can efficiently be parallelised, ${ }^{27}$ the current implementation is serial and all calculations were carried out on a single $2.8 \mathrm{GHz}$ Intel Core 5 processor $(8 \mathrm{~GB}$ memory), with the largest simulation taking roughly 1.5 days. For this reason, a thorough investigation of even larger systems, including an analysis of structure formation pathways, is left for the future.
A
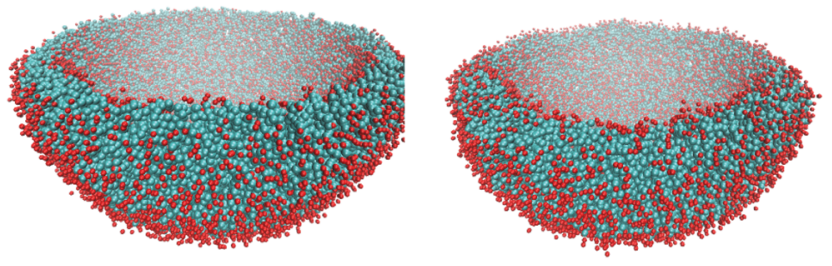

C
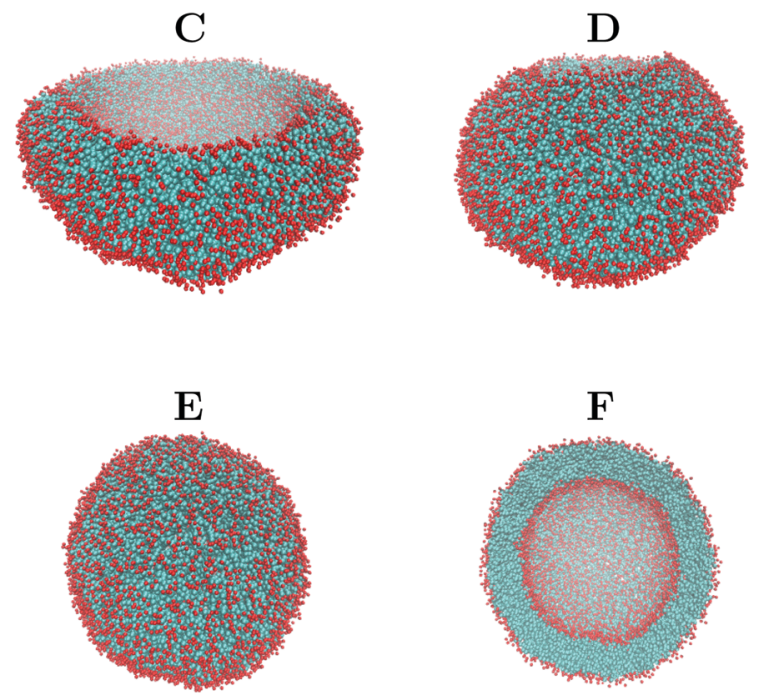

Fig. 26 Snapshots of a hybrid simulation in a $60 \times 60 \times 60$ simulation volume (all in units of $r_{\mathrm{C}}, n_{\mathrm{l}}=3986, N=648000, \Delta t^{*}=0.01$ ): after 200 (A), 2000 (B), 3000 (C), 10000 (D), 20000 (E) time steps and 20000 time steps where part of the vesicle is removed $(F)$, showing the structure of the bilayer. For clarity, only $\mathrm{H}_{3}\left(\mathrm{C}_{4}\right)_{2}$ lipids are shown, with red/blue representing $\mathrm{H} / \mathrm{C}$ particles.

\section{Conclusions}

In this work, we have combined the MD-SCF framework of Milano and Kawakatsu, ${ }^{26}$ which gains efficiency with respect to CGMD by replacing all non-bonded intermolecular interactions by a force derived from the density distribution or field of the ensemble of independent particles and particle chains, after particle-to-field projection, with the hybrid MPCD framework of Malevanets and Kapral, ${ }^{42}$ which combines a CGMD scheme for updating particle positions (streaming step) with a periodic random rotation of solvent particles in collision cells (collision step). Compared to the latter hybrid MPCD method, the main conceptual difference with the current approach is that the solvent also experiences solvent-solvent interactions, owing to the SCF-imposed compressibility in the solvent phase. Detailed analysis for pure solvent shows that this introduces a so-called caging effect at short times, reflected in an oscillating velocity auto-correlation function that exhibits regions of negative values, while the correct hydrodynamic scaling of MPCD was retained at longer times.

In most simulations, we start from an existing DPD lipid/ water representation and consider a discretisation distance $l$ of 
the field lattice equal to the cell size $a$ in MPCD, i.e. $l=a=r_{\mathrm{c}}$, the cut-off in DPD, but other representations and discretisation values are possible. Since we compared our results to standard DPD, with $\phi_{0}{ }^{*}=3$ or only three particles per lattice/rotation cell, there is clearly a lower bound for the field discretisation if we want to avoid quickly fluctuating fields. For molecular representations in the CGMD Martini framework, the particle density in the same volume is usually larger, and this is a lesser issue. Our projection places correctly normalised Gaussians around every particle, i.e. such that particle fractions that are projected to the lattice add up to one, instead of the original trilinear projection. This results in the desired smoother density field, as becomes apparent from the simulations for an ideal gas. The MPCD parameters $\alpha$ and $\delta t$, respectively the rotation angle in the collision step and the time step at which a collision is performed, can be used to tune effective hydrodynamic parameters, such as viscosity, in the solvent-rich domain. To perform $N V T$ simulations, a momentum-conserving thermostat was added, not only to the solvent but also to the solutes.

First, we tested previously derived mappings of DPD parameters to Flory-Huggins $\chi$ and Helfrich compressibility $\kappa_{\mathbf{H}}$ parameters that we need for the non-ideal free energy and chemical potential in MD-SCF/MPCD. By selecting a number of lipids relating to a tension-free flat DPD membrane in the same simulation volume, we were able to conclude, from the finding that a flat membrane is indeed formed for these hybrid parameters, that the mapping is consistent. In particular, since the surface tension is known as very sensitive to the average area per lipid, any mismatch will destabilise a flat membrane in favour of other structures, especially for the relatively small simulation volumes considered.

For the original MD-SCF method, which employs a global Andersen thermostat to maintain a constant simulation temperature, a defected tilted membrane forms for the same hybrid (thermodynamic) parameters, but we later showed that it can be attributed to the slightly anisotropic discrete gradient operator, which promotes certain directions on the computational lattice and gives rise to structure that is trapped in a metastable state. Reducing this anisotropy, the stable structure obtained by MD-SCF from the same initially homogeneous mixture is a flat membrane. In all cases, the phase separation dynamics in simulations with MD-SCF is orders of magnitude slower than with MD-SCF/MPCD, which we attributed, besides to the difference between explicit and implicit collisions in the two methods, to the fact that the Andersen thermostat is not Galilean invariant, which is known to affect dynamics.

We have also considered larger simulation volumes for the same (fixed) hybrid parameter set. For a volume $V^{*}=32^{3}$, containing approximately $10^{5}$ particles, we found that the stable structures are pathway dependent, starting from a lipid concentration consistent with a tensionless flat DPD membrane. A flat membrane is indeed formed when all lipids are initially randomly dispersed in only half of the volume, while alternative structures, with non-ideal free energies that are equivalent to that of the flat membrane, are formed from initially more dispersed systems. Since our research code is serial, we did not further increase system sizes for the initially fully mixed case, but we considered setups where part of the formation pathway was trespassed, i.e. starting from a curved platelet for $V^{*}=60^{3}$ or a punctured vesicle for $V^{*}=100^{3}$. The results, i.e. closing up into a vesicle for the curved platelet and the self-healing of the punctured vesicle, are fully consistent with expected mechanisms.

We note, however, that the MD-SCF/MPCD method lends itself perfectly to efficient parallellisation. Previous MD-SCF simulations were carried out using the parallel molecular dynamics suite OCCAM,${ }^{27}$ which can be easily adapted to incorporate MPCD and the improved discrete gradient operator. Since the kinetics is considerably accelerated by MPCD, and CGMD Martini representations are readily available for a wide range of biologically-relevant systems, one can efficiently simulate intricate phenomena in much larger systems than the current standard. For instance, MD-SCF/ MPCD allows one to extend a recent CGMD study of membrane binding/self-aggregation of fusogenic lipopeptides, embedded in a small membrane patch, ${ }^{57}$ to a realistic study of lipopeptide-induced fusion of vesicles of experimentally relevant sizes. Here, we have focussed on the foundations of this new hybrid methodology, and we leave such exercises for future studies.

\section{Appendix A: the particle-to-field projection}

Since the fields (and their derivatives) are defined on a cubic mesh and particles positions are essentially mesh-free, we need to define a projection $\hat{P}$ of particle fractions to mesh points. We start by dividing the simulation volume in $n_{x} \times n_{y} \times n_{z}$ cells (with $n_{i}, i \in\{x, y, z\}$, the number of cells in direction $i$ ) of size $l^{3}$. Periodic boundary conditions apply to this mesh. Using the particle coordinates $\mathbf{r}_{\mathrm{p}}=\left(x_{\mathrm{p}}, y_{\mathrm{p}}, z_{\mathrm{p}}\right)$ to extract the centre point $\left(i_{\mathrm{p}}, j_{\mathrm{p}}, k_{\mathrm{p}}\right)$ of the local 27-point mesh, with

$$
\begin{aligned}
i_{\mathrm{p}} & =\left[x_{\mathrm{p}} / l\right] \\
j_{\mathrm{p}} & =\left[y_{\mathrm{p}} / l\right] \\
k_{\mathrm{p}} & =\left[z_{\mathrm{p}} / l\right]
\end{aligned}
$$

and $[b]=\max \{m \in \mathbb{Z} \mid m \leq b\}$, particle fractions $w_{i}$ are distributed over this local mesh. To determine the fraction $w_{i}$ for each of the 27 mesh points, we position a Gaussian at the particle position $\mathbf{r}_{\mathrm{p}}$ (with spread $r_{\mathrm{c}}$ ) and calculate the function value at the local mesh points, making sure to conserve the total mass $\sum_{i=1}^{27} w_{i}=1$ by normalisation. The density field $\phi_{I}(i, j, k)$ is then constructed by summing over the particles of type $I$

$$
\phi_{I}(i, j, k)=\sum_{p=1}^{n_{\mathrm{p}}} \hat{P}\left(\mathbf{r}_{\mathrm{p}}\right) \quad \forall p \in I
$$

where $n_{\mathrm{p}}$ is the total number of particles. Since the resulting fields are a collection of truncated Gaussians, they are not necessarily differentiable. Nevertheless, the huge advantage over linear interpolation is that fields are considerably less sensitive to small particle displacements for a small particle density. Moreover, as the gradients are also calculated numerically using the same mesh, additional smoothing is not required. 
Field gradients are calculated via second order finite difference, on a staggered grid, as

$$
\begin{aligned}
\nabla_{x} \phi\left(i+\frac{1}{2}, j, k\right) & \approx \frac{1}{l}(\phi(i+1, j, k)-\phi(i, j, k)) \\
\nabla_{x} \phi\left(i-\frac{1}{2}, j, k\right) & \approx \frac{1}{l}(\phi(i, j, k)-\phi(i-1, j, k+1)) \\
\nabla_{y} \phi\left(i, j+\frac{1}{2}, k\right) & \approx \frac{1}{l}(\phi(i, j+1, k)-\phi(i, j, k)) \\
\nabla_{y} \phi\left(i, j-\frac{1}{2}, k\right) & \approx \frac{1}{l}(\phi(i, j, k)-\phi(i, j-1, k)) \\
\nabla_{z} \phi\left(i, j, k+\frac{1}{2}\right) & \approx \frac{1}{l}(\phi(i, j, k+1)-\phi(i, j, k)) \\
\nabla_{z} \phi\left(i, j, k-\frac{1}{2}\right) & \approx \frac{1}{l}(\phi(i, j, k)-\phi(i, j, k-1))
\end{aligned}
$$

and the staggered grid can be used directly to interpolate the density gradient at the particle position $\mathbf{r}_{\mathrm{p}}$, as

$$
\begin{aligned}
\nabla_{x} \phi\left(\mathbf{r}_{\mathrm{p}}\right)= & \left(\Delta x_{\mathrm{p}}+\frac{1}{2}\right) \nabla_{x} \phi\left(i_{\mathrm{p}}+\frac{1}{2}, j_{\mathrm{p}}, k_{\mathrm{p}}\right) \\
& +\left(\frac{1}{2}-\Delta x_{\mathrm{p}}\right) \nabla_{x} \phi\left(i_{\mathrm{p}}-\frac{1}{2}, j_{\mathrm{p}}, k_{\mathrm{p}}\right) \\
\nabla_{y} \phi\left(\mathbf{r}_{\mathrm{p}}\right)= & \left(\Delta y_{\mathrm{p}}+\frac{1}{2}\right) \nabla_{y} \phi\left(i_{\mathrm{p}}, j_{\mathrm{p}}+\frac{1}{2}, k_{\mathrm{p}}\right) \\
& +\left(\frac{1}{2}-\Delta y_{\mathrm{p}}\right) \nabla_{y} \phi\left(i_{\mathrm{p}}, j_{\mathrm{p}}-\frac{1}{2}, k_{\mathrm{p}}\right) \\
\nabla_{z} \phi\left(\mathbf{r}_{\mathrm{p}}\right)= & \left(\Delta z_{\mathrm{p}}+\frac{1}{2}\right) \nabla_{z} \phi\left(i_{\mathrm{p}}, j_{\mathrm{p}}, k_{\mathrm{p}}+\frac{1}{2}\right) \\
& +\left(\frac{1}{2}-\Delta z_{\mathrm{p}}\right) \nabla_{z} \phi\left(i_{\mathrm{p}}, j_{\mathrm{p}}, k_{\mathrm{p}}-\frac{1}{2}\right)
\end{aligned}
$$

with $\Delta x_{\mathrm{p}}=x_{\mathrm{p}} / l-\left[x_{\mathrm{p}} / l\right], \Delta y_{\mathrm{p}}=y_{\mathrm{p}} / l-\left[y_{\mathrm{p}} / l\right]$ and $\Delta z_{\mathrm{p}}=z_{\mathrm{p}} / l-\left[z_{\mathrm{p}} / l\right]$.

\section{Appendix B: alternative gradient stencils}

Instead of the efficient 2-point stencil for gradients along the three Cartesian directions, see the previous appendix, we may consider alternatives proposed in the recent literature. The development of isotropic discrete differential operators remains an important topic for many fields of research, as the inherent anisotropy associated with a computational grid implies a directional bias (in our case: to the forces stemming obtained from the mean-field external potential), which may, if not taken proper care off, be reflected in distinct grid-related artefacts and/or unphysical simulation results. Such undesired effects can be in most cases be sufficiently suppressed by considering additional points in the direct vicinity of a grid location and selecting stencil coefficients based on (particular) considerations of isotropy.
Here, we consider a recent gradient stencil developed for proper analysis of geophysical data, i.e. for images that are recorded on a regular grid of pixels, by Alfaraj et al. ${ }^{58}$ We note that the basics of these compact stencils is generic and that the particularity is in the choice of the stencil coefficients, which are subject to further improvement. Whereas their original stencil calculates gradient values on the same computational grid as the original data, here we use a denser grid and calculate gradient values on a staggered grid as before. Although this introduces the need for interpolation, it has the advantage of being more appropriate for spiked profiles, i.e. profiles with a small spacial extend or carrier. Since applying such gradient operators has the effect of smoothing, considering the original grid spacing would introduce the risk of mishandling these localised profiles. In particular, considering data points at only half the usual distance decreases the spatial extent over which two adjacent gradient values are calculated from 4 to 3 grid points.

In the most general form, the discrete gradient operator $G$ applied to a function $f$ at a position $\mathbf{r}=(x, y, z)$, can be written as a sum over all 27 grid-restricted directions

$$
G[f](\mathbf{r})=\sum_{i=-1}^{1} \sum_{j=-1}^{1} \sum_{k=-1}^{1} D_{i, j, k}(\mathbf{r})
$$

with

$$
D_{i, j, k}(\mathbf{r})=c_{i, j, k} \frac{f\left(\mathbf{r}+\frac{1}{2} \mathbf{r}_{i, j, k}\right)-f\left(\mathbf{r}-\frac{1}{2} \mathbf{r}_{i, j, k}\right)}{\left\|\mathbf{r}_{i, j, k}\right\| \cdot l}
$$

where $c_{i, j, k}$ are the stencil coefficients. Both $\mathbf{r}$ and $\mathbf{r}_{i, j, k}$ are defined in terms of the grid spacing $l$, hence the additional factor $l$ in the denominator. The stencil vectors $\mathbf{r}_{i, j, k}=i \cdot \mathbf{i}+j \cdot \mathbf{j}+k \cdot \mathbf{k}$ can be classified by their length $\left\|\mathbf{r}_{i, j, k}\right\|$ as

- 1 vector of length $0: i=j=k=0$

- 6 vectors of length $1:|i|+|j|+|k|=1$

- 12 vectors of length $\sqrt{2}:|i|+|j|+|k|=2$

- 8 vectors of length $\sqrt{3}:|i|+|j|+|k|=3$

It is easy to see that $c_{0,0,0}$ should be zero, which reduces the number of directions to 26. Moreover, since $\mathbf{r}_{-i,-j,-k}=-\mathbf{r}_{i, j, k}$, it is clear that the natural condition $D_{i, j, k}(\mathbf{r})=D_{-i,-j,-k}(\mathbf{r})$ provides the relation $c_{-i,-j,-k}=-c_{i, j, k}$. The number of independent stencil coefficients can thus simply be further reduced to 13 . Symmetry arguments are employed to even further reduce the number of independent variables to three, one for each vector length. We may take $c_{1,0,0}, c_{1,1,0}$ and $c_{1,1,1}$ as these independent variables.

To arrive at gradient stencils along Cartesian directions, i.e. $D_{x}, D_{y}$ and $D_{z}$, vectors without components along those directions are left out of the general expression (29), i.e. $c_{0, j, k}=0$ for $D_{x}, c_{i, 0, k}=0$ for $D_{y}$ and $c_{i, j, 0}=0$ for $D_{z}$. The expression can thus be simplified to

$$
\begin{aligned}
D_{x, y, z}= & c_{1}\left(f_{1}^{(1)}-f_{2}^{(1)}\right)+c_{2}\left(\sum_{i=1}^{4} f_{i}^{(2)}-\sum_{i=5}^{8} f_{i}^{(2)}\right) \\
& +c_{3}\left(\sum_{i=1}^{4} f_{i}^{(3)}-\sum_{i=5}^{8} f_{i}^{(3)}\right)
\end{aligned}
$$


in units of $1 / l$, where $c_{1}=2 c_{1,0,0}, \quad c_{2}=\sqrt{2} c_{1,1,0}$ and $c_{3}=2 c_{1,1,1} / \sqrt{3}$. For simplicity, we have introduced the shorthand notation of Thampi et al., ${ }^{59}$

$$
f_{i}^{(j)}=f\left(\mathbf{r}+\frac{1}{2} \mathbf{r}_{i}^{j}\right),
$$

with $j$ a counter for the previously introduced class of vectors of the same length (in units of the discretisation length $l$ ) in the first shell surrounding $\mathbf{r}$. Writing them explicitly for $D_{x, y, z}$ :

- $D_{x}: \mathbf{r}_{1}^{1}=(1,0,0)^{T}$ and $\mathbf{r}_{2}^{1}=-\mathbf{r}_{1}^{1} ; \mathbf{r}_{1}^{2}=(1,1,0)^{T}, \mathbf{r}_{2}^{2}=(1,-1,0)^{T}, \mathbf{r}_{3}^{2}=$ $(1,0,1)^{T}, \mathbf{r}_{4}^{2}=(1,0,-1)^{T}$, with $\mathbf{r}_{i+4}^{2}=-\mathbf{r}_{i}^{2}(1 \leq i \leq 4) ; \mathbf{r}_{1}^{3}=(1,1,1)^{T}, \mathbf{r}_{2}^{3}=$ $(1,1,-1)^{T}, \mathbf{r}_{3}^{3}=(1,-1,1)^{T}, \mathbf{r}_{4}^{3}=(1,-1,-1)^{T}$, with $\mathbf{r}_{i+4}^{3}=-\mathbf{r}_{i}^{3}(1 \leq i \leq 4)$.

- $D_{y}: \mathbf{r}_{1}^{1}=(0,1,0)^{T}$ and $\mathbf{r}_{2}^{1}=-\mathbf{r}_{1}^{1} ; \mathbf{r}_{1}^{2}=(1,1,0)^{T}, \mathbf{r}_{2}^{2}=(-1,1,0)^{T}, \mathbf{r}_{3}^{2}=$ $(0,1,1)^{T}, \mathbf{r}_{4}^{2}=(0,1,-1)^{T}$, with $\mathbf{r}_{i+4}^{2}=-\mathbf{r}_{i}^{2}(1 \leq i \leq 4) ; \mathbf{r}_{1}^{3}=(1,1,1)^{T}, \mathbf{r}_{2}^{3}=$ $(1,1,-1)^{T}, \mathbf{r}_{3}^{3}=(-1,1,1)^{T}, \mathbf{r}_{4}^{3}=(-1,1,-1)^{T}$, with $\mathbf{r}_{i+4}^{3}=-\mathbf{r}_{i}^{3}(1 \leq i \leq 4)$.

- $D_{z}: \mathbf{r}_{1}^{1}=(0,0,1)^{T}$ and $\mathbf{r}_{2}^{1}=-\mathbf{r}_{1}^{1} ; \mathbf{r}_{1}^{2}=(1,0,1)^{T}, \mathbf{r}_{2}^{2}=(-1,0,1)^{T}, \mathbf{r}_{3}^{2}=$ $(0,1,1)^{T}, \mathbf{r}_{4}^{2}=(0,-1,1)^{T}$, with $\mathbf{r}_{i+4}^{2}=-\mathbf{r}_{i}^{2}(1 \leq i \leq 4) ; \mathbf{r}_{1}^{3}=(1,1,1)^{T}, \mathbf{r}_{2}^{3}=$ $(1,-1,1)^{T}, \mathbf{r}_{3}^{3}=(-1,1,1)^{T}, \mathbf{r}_{4}^{3}=(-1,-1,1)^{T}$, with $\mathbf{r}_{i+4}^{3}=-\mathbf{r}_{i}^{3}(1 \leq i \leq 4)$.

An essential condition for the discrete gradient operators $D_{x, y, z}$ is correctness for linear functions $f(\mathbf{r})$ along the principal directions on the grid, so we may recast the still unknown stencil coefficients $\left\{c_{1}, c_{2}, c_{3}\right\}$ into another form $\left\{c_{1}, c_{2}, c_{3}\right\}=\left\{d_{1} /\left(d_{1}+4 d_{2}+\right.\right.$ $\left.\left.4 d_{3}\right), d_{2} /\left(d_{1}+4 d_{2}+4 d_{3}\right), d_{3} /\left(d_{1}+4 d_{2}+4 d_{3}\right)\right\}$, with $\left\{d_{1}, d_{2}, d_{3}\right\}$ a set of three random real numbers for which this condition is always satisfied. For completeness, we note that the normalisation factor $d_{1}+4 d_{2}+4 d_{3}$ is twice smaller than in Alfaraj et al. ${ }^{58}$ as a consequence of taking only half of the grid-restricted vector length. The freedom offered by these three new variables $d_{i}$ can be exploited to optimise the stencil towards accuracy (scaling behaviour) and isotropy, and we refer to a recent paper for the related discrete Laplacian for more information on the conditions that can be employed. ${ }^{60}$ The 2-point stencil in the previous Appendix uses the coefficients $\left\{d_{1}, d_{2}, d_{3}\right\}=\{1,0,0\}$. In their recent paper, Alfaraj et al. proposed the alternative coefficients $\left\{d_{1}, d_{2}, d_{3}\right\}=\{1,0.245,0.085\}$, which were obtained based on the condition of maximum isotropy for specific functions. ${ }^{58} \mathrm{We}$ select their stencil coefficients for the remainder.

The half-point values are now determined as

$$
\begin{aligned}
\nabla_{x} \phi\left(i+\frac{1}{2}, j, k\right) & \approx \frac{1}{l} D_{x} \phi\left(i+\frac{1}{2}, j, k\right) \\
\nabla_{x} \phi\left(i-\frac{1}{2}, j, k\right) & \approx \frac{1}{l} D_{x} \phi\left(i-\frac{1}{2}, j, k\right) \\
\nabla_{y} \phi\left(i, j+\frac{1}{2}, k\right) & \approx \frac{1}{l} D_{y} \phi\left(i, j+\frac{1}{2}, k\right) \\
\nabla_{y} \phi\left(i, j-\frac{1}{2}, k\right) & \approx \frac{1}{l} D_{y} \phi\left(i, j-\frac{1}{2}, k\right) \\
\nabla_{z} \phi\left(i, j, k+\frac{1}{2}\right) & \approx \frac{1}{l} D_{z} \phi\left(i, j, k+\frac{1}{2}\right) \\
\nabla_{z} \phi\left(i, j, k-\frac{1}{2}\right) & \approx \frac{1}{l} D_{z} \phi\left(i, j, k-\frac{1}{2}\right)
\end{aligned}
$$

where the off-grid values of the density fields $\phi$ are obtained by linear interpolation of the (two or four) surrounding field values on the computational grid. As before, see previous Appendix, the staggered grid can be used directly to interpolate the density gradient at the particle position $\mathbf{r}_{\mathrm{p}}$.

\section{Appendix C: hydrodynamics}

One may claim that the field-based interactions in the MD-SCF part of the MD-SCF/MPCD scheme necessarily break Galilean invariance, because they make an explicit reference to the underlying (field) mesh. Although this is primarily a technical issue in the development of hybrid particle/field methodologies like MD-SCF, i.e. it does not relate to the coupling to MPCD discussed in this study, one may be tempted to conclude that there is no momentum conservation or hydrodynamic interaction (in the common sense). The MD-SCF/MPCD scheme indeed does not rigorously enforce actio=reactio in the non-bonded part of the particle forces and, as a consequence, local momentum may not be strictly conserved. Yet, it is unclear to us how we can evaluate to what extent the continuity equation for the momentum $(\nabla \cdot \mathbf{v}=0)$ is violated without the use of a computational mesh. On the other hand, monitoring the total momentum in our simulations is straightforwards and relevant, since conservation of local momentum implies that also the total momentum is conserved. As our scheme represents a discrete form of a continuum theory, which is strictly momentum conserving, one may assume that hydrodynamic interactions will come out correctly, apart from errors due to discretisation. To substantiate our claim that our scheme correctly describes hydrodynamic interactions, we therefore focus on the necessary condition, i.e. whether our scheme conserves total momentum.

We analyse total momentum via the previously considered small system, i.e. a cubic volume $V^{*}\left(L_{x, y, z^{*}}=22\right)$ containing $n_{1}=764$ lipids and $n_{\mathrm{w}}=23540$ solvent particles, where we take all other parameters as before. Since the mass $m^{*}$ of all particles is set to unity, the dimensionless total momentum $\left\langle\mathbf{p}^{*}\right\rangle=\sum_{p} \mathbf{p}_{p}$ (with $p=1, \ldots, N$ the particle index) is given by

$$
\left\langle\mathbf{p}^{*}\right\rangle=\left\langle\mathbf{v}^{*}\right\rangle=\left(\left\langle v_{x}^{*}\right\rangle,\left\langle v_{y}^{*}\right\rangle,\left\langle v_{z}^{*}\right\rangle\right)^{T}
$$

In comparison, the conservative forces in the DPD, which are derived from a translationally invariant Hamiltonian, as well as the dissipative and stochastic forces, give rise to particle momenta that exactly sum up to zero along the three Cartesian directions, and also for standard MPCD, conservation of total momentum is guaranteed by construction. Analysing the membrane forming system in the same volume $V^{*}$ (snapshots shown in Fig. 7) for Galilean invariant DPD reveals that the three components of the total momentum $\left\langle\mathbf{p}^{*}\right\rangle$ fluctuate along the simulation trajectory between 0 and $-6 \times 10^{-16}$, i.e. in agreement with the machine epsilon of $2.22 \times 10^{-16}$ for double precision. For the hybrid MD-SCF/MPCD simulations, we consider four different setups: for the compact (old) and the isotropic (new) gradient operator, and with or without a constant background velocity $\mathbf{v}_{\mathrm{c}}$ added to all particles, after standard initialisation of particle velocities from the Maxwell-Boltzmann distribution for $T^{*}=1$. Adding a constant background velocity will change the inertial 
frame and provides information about the effect of the (stationary) field mesh and the MPCD cells on the particle forces/momenta; we refer to this setup as moving frame and to the standard case without background velocity as fixed frame. In case of Galilean invariance, the system behaviour will be invariant under this transformation. We analyse results obtained for $\mathbf{v}_{\mathrm{c}}{ }^{*}=\left(v_{\mathrm{c}}, 0,0\right)$, with $v_{\mathrm{c}}$ a random value drawn from the same Maxwell-Boltzmann distribution, but also background velocities along the other Cartesian directions were tested, with equivalent results.

Fig. 27 shows the evolution of the mean-field free energy, the simulation temperature $T_{\text {sim }}$ (with $T_{\text {sim }}=\left\langle\left(\mathbf{v}^{*}\right)^{2}\right\rangle / 3$ ), and one of the components of the total momentum $\left\langle\mathbf{p}^{*}\right\rangle$ for the four simulated setups. To enable a direct comparison for fixed and moving frames, the momentum contribution of the background velocity has been subtracted in the calculation procedure for the total momentum (but not in the calculation of $\left.T_{\text {sim }}\right)$.

The mean-field free energy plots, see Fig. 27A, show that the trajectories slightly differ between setups, in particular that they are sensitive to the choice of the discrete gradient operator. With respect to the fixed or moving frames, it makes sense that adding a background velocity slightly perturbs the particular trajectory, as a consequence of the static computational grids employed in the particle-to-field projection and the MPCD collision step, but the overall behaviour should be very similar. Indeed, both visual analysis of final stages and the equivalent plateau values for the mean-field free energy show that a stable flat membrane self-assembles for all considered setups (e.g. snapshot in Fig. 7). Apart from an affine shift due to the (constant) background velocity, also the evolution of the simulation temperature is rather insensitive to the choice of the setup. The components of the total momentum - Fig. 27 only shows the $z$-component, but the amplitudes of the other components are comparable for a fixed frame, see extended discussion later on - which should remain close to the machine epsilon if conserved, are also comparable for every particular setup. Yet, although the time-averaged value of the total momentum is close to vanishing, the amplitude of the fluctuations is many orders higher $\left(\sim 10^{-3}\right)$ than the machine epsilon $\left(\sim 10^{-16}\right)$. Nevertheless, a closer look shows that the total momentum drops to the level of the machine epsilon after every collision step.

It is a common procedure in MPCD to remove any net momentum generated by rounding errors during the collision step, by determining the (vectorial) mean of the velocity distribution and subtracting it from the velocity for each particle. The same procedure is always applied after initialisation of the particle velocities, both in MD-SCF/MPCD and in DPD. Resetting the mean of the velocity distribution to zero is particularly required to avoid any non-physical drift during simulation. If we extend this procedure also to the inner loop in MD-SCF/ MPCD, i.e. the VV scheme, for all considered setups, we obtain the data shown in Fig. 28. Concentrating on the total momentum, we see that the amplitude of the fluctuations has decreased dramatically, to the level of the machine epsilon.
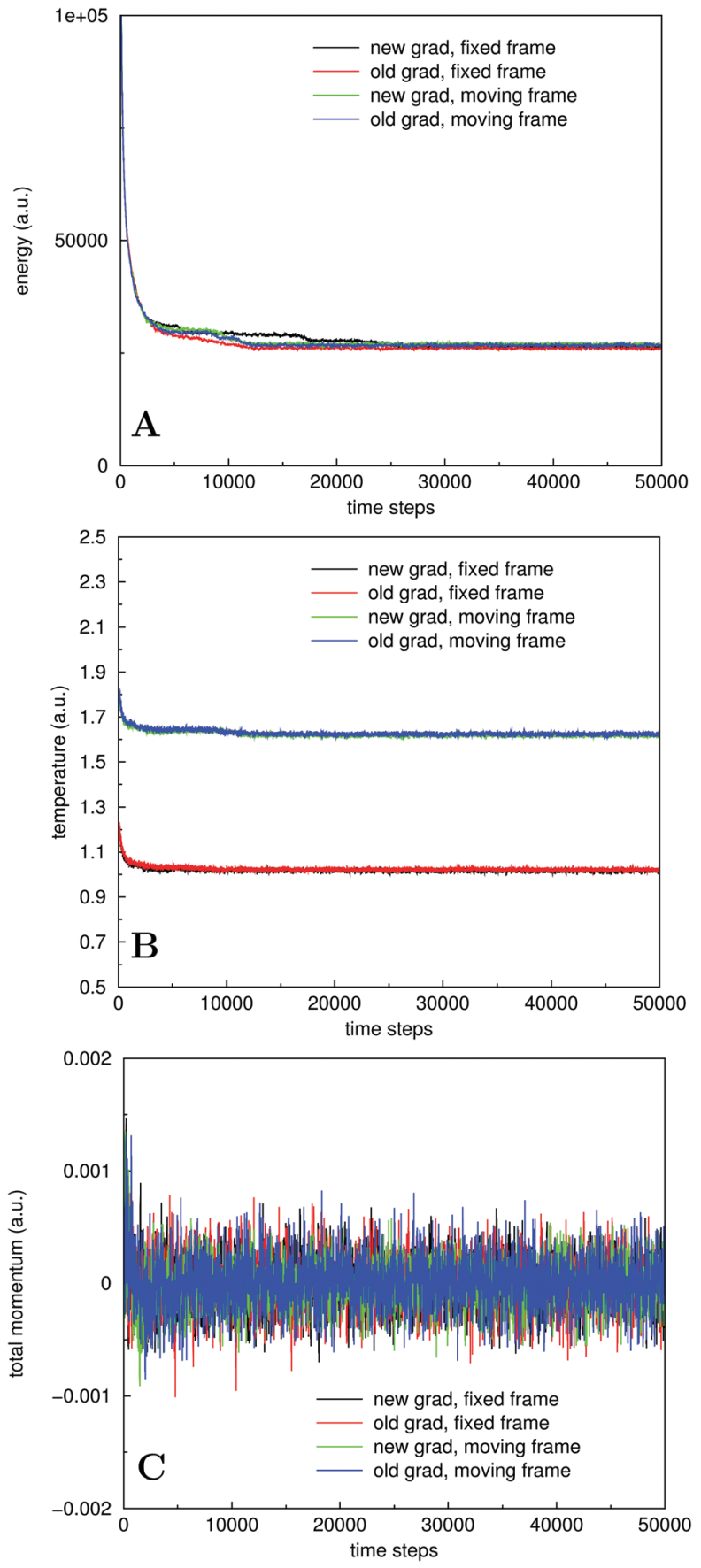

Fig. 27 The SCF free energy (A), kinetic temperature (B) and total momentum ( $C, z$-component) as determined by MD-SCF/MPCD for the four considered setups. A snapshot of the stable structure for the old gradient operator and fixed frame is shown in Fig. 7. Equivalent flat membranes are found as stable structure for all setups. For the two setups with moving frames, the momentum contribution of the background velocity has been subtracted in the calculation of the total momentum.

The removal of net momentum does not notably affect the evolution of the simulation temperature. The trajectories, as reflected in the evolution of the mean-field free energy, again display slightly different features. For the moving frame, the signatures are comparable to the ones without removal of net 

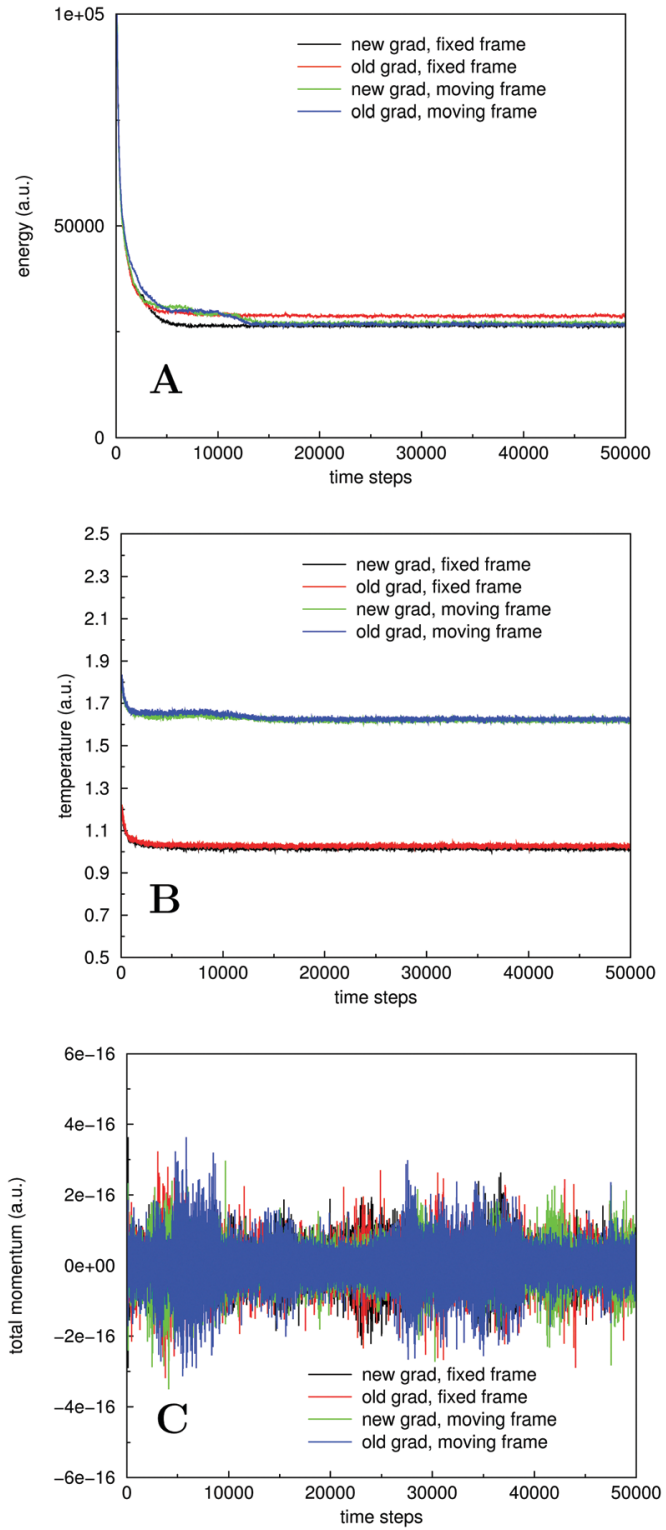

Fig. 28 The SCF free energy (A), kinetic temperature (B) and total momentum ( $C, z$-component) as determined by MD-SCF/MPCD with removal of net momentum at each step for the four considered setups. Only for the old gradient operator and a fixed frame, the final state is not a flat membrane, but a metastable structure shown in Fig. 29, which is usually found as a transient state. For the two setups with moving frames, the momentum contribution of the background velocity has been subtracted in the calculation of the total momentum.

momentum. For the fixed frame and a slightly anisotropic (old) gradient operator, the system gets stuck in a metastable state of an interconnected membrane, see Fig. 29 which is reminiscent of a transient structure in the other simulations. Instead, for a fixed frame and a more isotropic gradient operator, phase separation into a defect-free planar membrane is rather swift and accelerated compared to the case when net momentum was not removed. We conclude that, in the anisotropic case, adding a fluctuating tiny net momentum apparently helps the system to cross the energetic barrier between this structure and

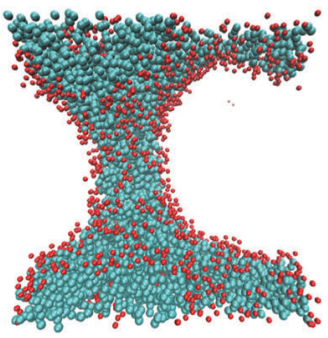

Fig. 29 The metastable structure obtained for the old gradient operator and a fixed frame, see Fig. 28. For clarity, only $\mathrm{H}_{3}\left(\mathrm{C}_{4}\right)_{2}$ lipids are shown, with red/blue representing $\mathrm{H} / \mathrm{C}$ particles.

the equilibrium planar membrane. Indeed, also for the MD-SCF method with an Anderson thermostat, a similar effect was observed after the introduction of a more isotropic gradient operator.

We may analyse the effects of discretisation in detail by comparing the total momentum along and perpendicular to the direction in which the reference frame moves with constant velocity, see Fig. 30 for the isotropic (new) gradient operator. Clearly, the amplitude of the total momentum along the $x$-direction in which the frame moves, $\left\langle p_{x}\right\rangle$, is about a factor $10^{2}$ larger (but, in absolute terms, still tiny, $\sim 10^{-14}$ ) than the amplitude for the total momentum along the perpendicular directions $\left(\left\langle p_{y}\right\rangle\right.$ and $\left.\left\langle p_{z}\right\rangle\right)$, which are comparable to the machine epsilon, see Fig. 28. Analysis of other setups shows that this is relation is representative. Although this is clearly due to discretisation errors, they cannot be attributed specifically to either one of the underlying coarser representations; a more detailed theoretical analysis is left for the future. It only shows that Galilean invariance is indeed broken, albeit only rather mildly.

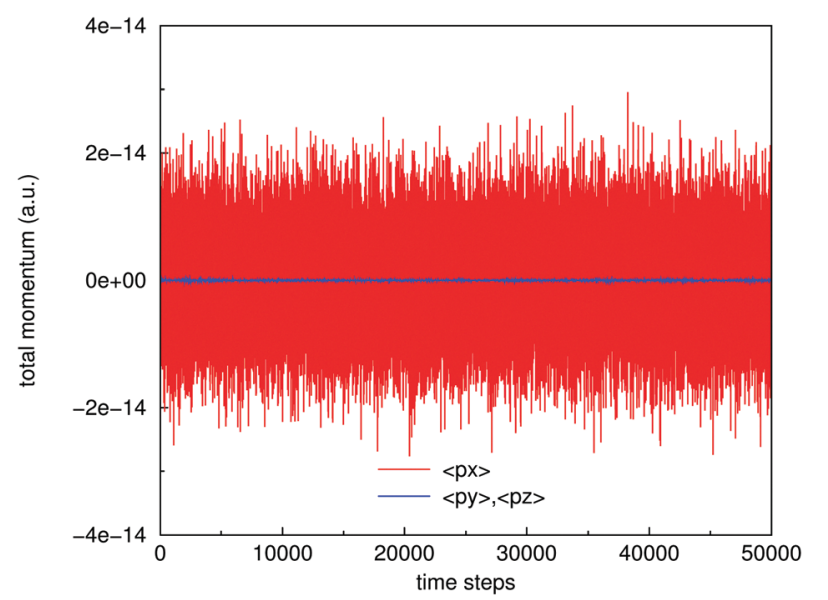

Fig. 30 All three components of total momentum as determined by MD-SCF/MPCD for the new (isotropic) gradient operator and a moving frame. These data, which are representative for the case where the frame moves, show the difference in total momentum along (here: $x$-direction) or perpendicular $(y, z$-directions) to the movement of the frame. For a fixed frame, the amplitude of all three components is comparable and equal to the machine epsilon $\left(\sim 10^{-16}\right)$. The momentum contribution of the background velocity has been subtracted in the calculation of the total momentum. 
Overall, we conclude that the particles in the 'unscaled' MD-SCF/MPCD scheme experience a tiny shaking each time step, which indeed averages out on longer time scales, corroborating our earlier statement that the hydrodynamic interactions will come out correctly on a coarser time scale. Removing any net momentum at every step of the MD-SCF/MPCD scheme gives rise to total momentum conservation, which is exact up to machine precision. In short, we have shown that our model indeed properly captures hydrodynamic interactions.

\section{Acknowledgements}

GJAS is very grateful to Ignacio Pagonabarraga and Edgar Blokhuis for stimulating discussions and very helpful comments. GJAS and FS acknowledge funding by the VW foundation in the context of an international project Multiscale hybrid modeling of (bio)membranes. FS also acknowledges partial support by the German Science Foundation, SFB TRR 146 (project C1).

\section{References}

1 B. J. Alder and T. E. Wainwright, J. Chem. Phys., 1959, 31, 459.

2 A. Rahman, Phys. Rev., 1964, 136, A405.

3 B. Smit, P. A. J. Hilbers, K. Esselink, L. A. M. Rupert, N. M. van Os and A. G. Schlijper, Nature, 1990, 348, 624.

4 P. J. Hoogerbrugge and J. M. V. A. Koelman, Europhys. Lett., 1992, 19, 155.

5 J. F. Dama, A. V. Sinitskiy, M. McCullagh, J. Weare, B. Roux, A. R. Dinner and G. A. Voth, J. Chem. Theory Comput., 2013, 9, 2466.

6 A. Davtyan, J. F. Dama, A. V. Sinitskiy and G. A. Voth, J. Chem. Theory Comput., 2014, 10, 5265.

7 S. J. Marrink, H. J. Risselada, S. Yefimov, D. P. Tieleman and A. H. de Vries, J. Phys. Chem. B, 2007, 111, 7812.

8 G. J. A. Sevink and A. V. Zvelindovsky, Macromolecules, 2005, 38, 7502 .

9 T. Uneyama and M. Doi, Macromolecules, 2007, 126, 114902.

10 X. H. He and F. Schmid, Phys. Rev. Lett., 2008, 100, 137802.

11 L. Zhang, G. J. A. Sevink and F. Schmid, Macromolecules, 2011, 44, 9434.

12 M. Muller, K. Katsov and M. Schick, Phys. Rep., 2006, 434, 113.

13 T. Kawakatsu, K. Kawasaki, M. Furusaka, H. Okabayashi and T. Kanaya, J. Phys.: Condens. Matter, 1994, 6, 6385.

14 G. J. A. Sevink, M. Charlaganov and J. G. E. M. Fraaije, Soft Matter, 2013, 8, 5102.

15 Z.-J. Wang and M. Deserno, New J. Phys., 2010, 12, 095004.

16 Z.-J. Wang and M. Deserno, J. Phys. Chem. B, 2010, 114, 11207.

17 G. J. A. Sevink and J. G. E. M. Fraaije, Soft Matter, 2014, 10, 5129 .

18 M. Deserno, Macromol. Rapid Commun., 2009, 30, 752.

19 K. Pluhackova and R. A. Böckmann, J. Phys.: Condens. Matter, 2015, 27, 323103.
20 Y. Wang, J. K. Sigurdsson, E. Brandt and P. J. Atzberger, Phys. Rev. E: Stat., Nonlinear, Soft Matter Phys., 2013, 88, 023301.

21 R. D. Groot and P. B. Warren, J. Chem. Phys., 1997, 107, 4423.

22 M. Müller and G. D. Smith, J. Polym. Sci., Part B: Polym. Phys., 2005, 43, 934.

23 K. Ch. Daoulas, M. Müller, J. J. de Pablo, P. F. Nealey and G. D. Smith, Soft Matter, 2006, 2, 573.

24 M. Hömberg and M. Müller, J. Chem. Phys., 2010, 132, 155104.

25 M. Hömberg and M. Müller, Europhys. Lett., 2012, 97, 68010.

26 G. Milano and T. Kawakatsu, J. Chem. Phys., 2009, 130, 214106

27 Y. Zhao, A. De Nicola, T. Kawakatsu and G. Milano, J. Comput. Chem., 2012, 33, 868.

28 M. Winger, D. Trzesniak, R. Baron and W. F. van Gunsteren, Phys. Chem. Chem. Phys., 2009, 11, 1934; S. J. Marrink, X. Periole, D. P. Tieleman and A. H. de Vries, Phys. Chem. Chem. Phys., 2010, 12, 2254.

29 A. de Nicola, Y. Zhao, T. Kawakatsu, D. Roccatano and G. Milano, J. Chem. Theory Comput., 2011, 7, 2947.

30 S. D. Stoyanov and R. D. Groot, J. Chem. Phys., 2005, 122, 114112.

$31 \mathrm{H}$. Noguchi and G. Gompper, Europhys. Lett., 2007, 79, 36002 .

32 H. Noguchi, K. Kikuchi and G. Gompper, Europhys. Lett., 2007, 78, 10005.

33 A. Lamura, G. Gonella and J. M. Yeomans, Europhys. Lett., 1999, 45, 314.

34 J. C. Desplat, I. Pagonabarraga and P. Bladon, Comput. Phys. Commun., 2001, 134, 273.

35 T. Ihle and D. M. Kroll, Phys. Rev. E: Stat., Nonlinear, Soft Matter Phys., 2003, 67, 066705.

36 E. Allahyarov and G. Gompper, Phys. Rev. E: Stat., Nonlinear, Soft Matter Phys., 2002, 66, 036702.

37 T. Ihle, E. Tüzel and D. M. Kroll, Phys. Rev. E: Stat., Nonlinear, Soft Matter Phys., 2004, 70, 035701.

38 T. Ihle, E. Tüzel and D. M. Kroll, Phys. Rev. E: Stat., Nonlinear, Soft Matter Phys., 2005, 72, 046707.

39 A. Malevanets and J. M. Yeomans, Europhys. Lett., 2000, 52, 231.

40 E. Tüzel, G. Pan, T. Ihle and D. M. Kroll, Europhys. Lett., 2007, 80, 40010.

41 Y. Hashimoto, Y. Chen and H. Ohashi, Comput. Phys. Commun., 2000, 129, 56.

42 A. Malevanets and R. Kapral, J. Chem. Phys., 2000, 112, 7260.

43 C.-C. Huang, A. Varghese, G. Gompper and R. G. Winkler, Phys. Rev. E: Stat., Nonlinear, Soft Matter Phys., 2015, 91, 013310.

44 J. C. Shillcock and R. Lipowsky, J. Chem. Phys., 2002, 117, 5048.

45 B. Leimkuhler and X. Shang, J. Comp. Physiol., 2015, 280, 72. 46 G. Gompper, T. Ihle, D. M. Kroll and R. G. Winkler, $A d v$. Polym. Sci., 2009, 221, 1.

47 M. Ripoll, K. Mussawisade, R. G. Winkler and G. Gompper, Phys. Rev. E: Stat., Nonlinear, Soft Matter Phys., 2005, 72, 016701. 
48 M. H. Ernst, E. H. Hauge and J. M. J. van Leeuwen, Phys. Rev. A: At., Mol., Opt. Phys., 1971, 4, 2055.

49 A. Rahman and F. H. Stillinger, J. Chem. Phys., 1971, 55, 3336.

50 J. T. Padding and A. A. Louis, Phys. Rev. E: Stat., Nonlinear, Soft Matter Phys., 2006, 74, 031402.

51 G. J. A. Sevink, in Nanostructured Soft Matter, ed. A. V. Zvelindovsky, 2007, p. 269.

52 S. T. Hyde, Langmuir, 1997, 13, 842.

53 K. Michielsen and H. De Raedt, Phys. Rep., 2001, 347, 462.

54 K. Michielsen, H. De Raedt and J. G. E. M. Fraaije, Prog. Theor. Phys. Suppl., 2000, 138, 543.
55 A. Grafmüller, J. Shillcock and R. Lipowsky, $P R L, 2007$, 98, 218101.

56 W. Shinoda, T. Nakamura and S. O. Nielsen, Soft Matter, 2011, 7, 9012.

57 M. Bulacu and G. J. A. Sevink, Biochim. Biophys. Acta, 2014, 1848, 848.

58 M. Alfaraj, Y. Wang and Y. Luo, Geophys. Prospect., 2014, 62, 507.

59 S. P. Thampi, S. Ansumali, R. Adhikari and S. Succi, J. Comp. Physiol., 2013, 234, 1.

60 G. J. A. Sevink, Phys. Rev. E: Stat., Nonlinear, Soft Matter Phys., 2015, 91, 053309. 